Empacotamento de subgrafos em grafos

\author{
Gordana Manić
}

TESE APRESENTADA

AO

INSTITUTO DE MATEMÁTICA E ESTATÍSTICA

DA

UNIVERSIDADE DE SÃO PAULO

PARA

OBTENÇÃO DE GRAU DE DOUTOR

EM

CIÊNCIAS

Área de Concentração: Ciência da Computação

Orientadora: Profa. Dra. Yoshiko Wakabayashi

Durante a elaboração deste trabalho a autora recebeu apoio financeiro da CAPES

São Paulo, 8 de agosto de 2006 


\section{Empacotamento de subgrafos em grafos}

Este exemplar corresponde à redação final da tese devidamente corrigida e defendida por Gordana Manić

e aprovada pela Comissão Julgadora.

São Paulo, 30 de março de 2007

Banca Examinadora:

Profa. Dra. Yoshiko Wakabayashi (orientadora) - IME/USP

Prof. Dr. José Coelho de Pina Júnior - IME/USP

Prof. Dr. Flávio Keidi Miyazawa - IC/UNICAMP

Prof. Dr. Luerbio Faria - DMAT/UERJ

Prof. Dr. Marcelo Henriques de Carvalho - CCET/UFMS 
À minha mãe, ao meu irmão e à memória de meu pai com afeto e estima. 


\section{Agradecimentos}

À minha orientadora, professora Yoshiko Wakabayashi, pela sua excelente orientação, paciência e dedicação a este trabalho. Aos demais professores do grupo de Combinatória, por toda ajuda na minha formação acadêmica.

Aos meus pais e irmão, pelo incentivo incondicional em todas as minhas iniciativas, depositando em mim a confiança necessária para enfrentar qualquer dificuldade, e me dando o prazer de compartilhar com eles a alegria das minhas conquistas (Mojim roditeljima $i$ bratu, za podrsku i neophodno poverenje u svim mojim inicijativama. Njima dugujem za sve postignute uspehe).

Ao professor Fernando Cortez Sica de Ouro Preto, ao Goroljub e Monica Milošević e a senhora Ivanka Ajdarić de Salvador que me incentivaram a ingressar no IME-USP, pelo apoio que me deram na ocasião, num dos momentos críticos. Ao senhor Dragiša Pavličić que me ajudou assim que cheguei à cidade de São Paulo.

Ao Cardonha, pelas conversas, apoio e amizade. Ao Frédéric Chataigner, pelas dicas e ajuda em diversas partes da minha tese, e ao Marcel Kenji pela leitura, correções e auxílio durante a redação deste texto.

Aos amigos desde os tempos do mestrado, Orlando Lee, Mário Leston, Pil e Renato de Carmo. A Maya pelos passeios, e aos demais amigos da salinha "vip": Domingos Soares, Domingos Jr., Marcelo, Ellen, Luna, Cris, Fabrício, Trevizan e Ju, que juntos tornaram o meu dia-a-dia mais agradável. Ao Maurício, Geraldine, Thomas, Lilian e à galera da "salinha do café".

À Nevena e ao Igor, Joca, Milan Lalić, Marijana e Pedja, Ana e Ivan, pela amizade e bate-papos indispensáveis em sérvio.

À Kémelli e à Claudete pela amizade, simpatia e pelos bons tempos que passamos juntos.

Aos amigos da minha cidade natal, Novi Sad, que mesmo estando longe são sempre uma constante fonte de energia e amizade: Borislava Ilić, Neda Divjaković e Ana Grubor.

Aos amigos Llane Alexis, Rino Carvalho, Maria de Souza e à moçada de Salvador, pelas férias inesquecíveis. 


\section{Resumo}

Dentre os problemas clássicos da área de otimização combinatória distingüem-se os chamados problemas de empacotamento, que variam de acordo com os objetos a serem empacotados e as funções a serem otimizadas. Quando os objetos de interesse são grafos, vários desses problemas podem ser classificados como problemas de $\mathcal{F}$-empacotamento, onde $\mathcal{F}$ é uma família de grafos. Estes problemas são assim definidos. Dado um grafo $G$, encontrar em $G$ um subgrafo $H$ que seja uma união de grafos disjuntos nos vértices (ou nas arestas), cada um dos quais é isomorfo a algum grafo da família $\mathcal{F}$, e tal que $H$ tenha o maior número possível de vértices (ou arestas). Temos assim várias variantes, dependendo se consideramos disjunção nos vértices ou nas arestas, e se queremos maximizar o número de vértices ou arestas de $H$.

Um problema bem conhecido desse tipo é o problema do emparelhamento máximo, que corresponde ao caso em que $\mathcal{F}=\left\{K_{2}\right\}$ e $H$ deve consistir de subgrafos disjuntos nos vértices. Nesta tese investigamos os casos $\mathcal{F}=\left\{K_{3}\right\}$ e $\mathcal{F}=\left\{K_{2}, K_{3}\right\}$, com ênfase no projeto de algoritmos de aproximação e resultados de inaproximabilidade.

No caso $\mathcal{F}=\left\{K_{3}\right\}$, estudamos o problema em que $H$ deve ser uma união de triângulos disjuntos nos vértices, denotado por VTP, e o problema em que $H$ deve ser uma união de triângulos disjuntos nas arestas e o objetivo é maximizar o número de arestas de $H$, denotado por ETP. Ambos os problemas são NP-difíceis. A melhor razão de aproximação conhecida para os dois problemas é $3 / 2+\varepsilon$, obtida por Hurkens e Schrijver em 1989. Apresentamos melhoras na razão de aproximação para casos restritos dos problemas VTP e ETP que são sabidamente APX-difíceis: apresentamos um algoritmo de aproximação para o VTP em grafos de grau máximo 4 com razão um pouco menor que 1,2, e para o ETP em grafos de grau máximo 5 com razão 4/3. Apresentamos ainda um algoritmo linear exato para o VTP em grafos de intervalos unitários.

No caso em que $\mathcal{F}=\left\{K_{2}, K_{3}\right\}$, estudamos o problema em que os subgrafos de $H$ devem ser disjuntos nos vértices e $H$ deve ter o maior número possível de arestas. Provamos que este problema é APX-difícil mesmo em grafos de grau máximo 4. Ademais, apresentamos uma $(3 / 2+\varepsilon)$-aproximação para grafos arbitrários e uma 1,4 -aproximação para grafos de grau máximo 4.

Palavras-chaves: algoritmos de aproximação. algoritmos polinomiais, APX-difícil, empacotamento de arestas, empacotamento de triângulos, grafos de intervalos unitários, grau limitado. 


\begin{abstract}
A classical problem in graph theory is the problem of finding a maximum matching in a graph. This problem can be seen as an $\mathcal{F}$-packing problem, where $\mathcal{F}$ is a family of graphs. This problem is defined as follows. Given a graph $G$, find a subgraph $H$ in $G$ that is a union of vertex-disjoint (or edge-disjoint) graphs, each of which is isomorphic to a graph in $\mathcal{F}$, and such that $H$ has a maximum possible number of vertices (or edges). We may have several variants of this problem, according to the restrictions on $H$ (vertex or edge disjoint version) and on the objective function.

In this thesis we investigate the cases in which $\mathcal{F}=\left\{K_{3}\right\}$ and $\mathcal{F}=\left\{K_{2}, K_{3}\right\}$, seeking approximation algorithms and inapproximability results.

When $\mathcal{F}=\left\{K_{3}\right\}$, we study the vertex-disjoint version, denoted here VTP, and the edgedisjoint version with the objective of maximizing the number of edges of $H$, denoted ETP. Both problems are NP-hard. The algorithm with the best approximation ratio known so far for these problems has ratio $3 / 2+\varepsilon$, a result obtained by Hurkens and Schrijver in 1989 . We present improvements on the approximation ratio for restricted cases of VTP and ETP that are known to be APX-hard: we give an approximation algorithm for VTP on graphs with maximum degree 4 with ratio slightly less than 1.2, and for ETP on graphs with maximum degree 5 with ratio $4 / 3$. We also present an exact linear-time algorithm for VTP on the class of unit interval graphs.

When $\mathcal{F}=\left\{K_{2}, K_{3}\right\}$, we study the vertex-disjoint version with the objective of maximizing the number of edges of $H$. We prove that this problem is APX-hard even on graphs with maximum degree 4 . Furthermore, we present a $(3 / 2+\varepsilon)$-approximation algorithm for arbitrary graphs, and a 1.4-approximation algorithm for graphs with maximum degree 4 .
\end{abstract}

Keywords: approximation algorithm, APX-hardness, edge packing, graph, low degree, polynomial algorithm, triangle packing, unit interval graph 


\section{Sumário}

1 Introdução 1

1.1 Empacotamento de subgrafos em grafos $\ldots \ldots \ldots \ldots \ldots \ldots$

2 Preliminares 5

2.1 Teoria dos Grafos . . . . . . . . . . . . . . . . . . . . . 5

2.2 Conjuntos e problemas de empacotamento . . . . . . . . . . . . . 6

2.3 Classes de problemas, algoritmos e complexidade . . . . . . . . . . . 7

3 Empacotamento de triângulos disjuntos nos vértices 9

3.1 Introdução . . . . . . . . . . . . . . . . . . . . . . . . . 9

3.2 Grafos arbitrários . . . . . . . . . . . . . . . . . . 10

3.2 .1 Algoritmo de Hurkens e Schrijver . . . . . . . . . . . . . . . . . 10

3.3 Grafos com grau máximo $4 \ldots \ldots \ldots \ldots \ldots \ldots$

3.3 .1 Introdução . . . . . . . . . . . . . . . . . . . 15

3.3 .2 Algoritmo de aproximação $\mathrm{VT}_{k} \ldots \ldots \ldots \ldots$. . . . . . . . 17

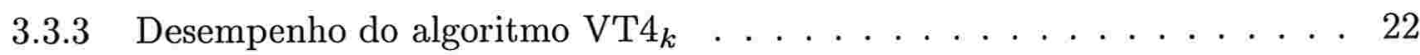

3.3.4 Complexidade computacional do algoritmo $\mathrm{VT}_{k} \ldots \ldots \ldots$. . . . . . . 32

3.3 .5 Observações finais . . . . . . . . . . . . . . . . . . 33

3.4 Grafos de intervalos unitários . . . . . . . . . . . . . . . . 35

3.4 .1 Introdução . . . . . . . . . . . . . . . . . . . 35

3.4 .2 Algoritmo exato . . . . . . . . . . . . . . 35

3.4 .3 Observações finais . . . . . . . . . . . . . . . 36

4 Empacotamento de triângulos disjuntos nas arestas 38

4.1 Introdução. . . . . . . . . . . . . . . . . . 38

4.2 Grafos arbitrários . . . . . . . . . . . . . . . . . . 38

4.3 Grafos com grau máximo $5 \ldots \ldots \ldots \ldots \ldots$

4.3 .1 Algoritmo de aproximação ET5 . . . . . . . . . . . . . . . . . . . . . . . . . . . . . 39

4.3 .2 Desempenho do algoritmo ET5 . . . . . . . . . . . . . . . 39

4.3.3 Complexidade computacional do algoritmo ET5 . . . . . . . . . . . . 41 
5 Empacotamento de arestas e triângulos 42

5.1 Introdução . . . . . . . . . . . . . . . . . . . . . . . . . 42

5.2 Resultados de inaproximabilidade . . . . . . . . . . . . . . . . . . . . . . . . . . . . 50

5.3 Algoritmo de aproximação . . . . . . . . . . . . . . . . . . . . . 50

5.4 Observações finais . . . . . . . . . . . . . . . . . 53

6 Considerações finais $\quad 56$

$\begin{array}{ll}\text { Referências Bibliográficas } & 57\end{array}$

$\begin{array}{lr}\text { Índice Remissivo } & 60\end{array}$ 


\section{Capítulo 1}

\section{Introdução}

Muitos problemas de otimização combinatória de grande importância e vasta aplicação prática são reconhecidamente NP-difíceis. Para esses problemas, os algoritmos conhecidos que encontram soluções ótimas consomem tempo exponencial, o que na prática é inviável. Em função do grande número e da importância desses problemas, diversas abordagens têm sido desenvolvidas na tentativa de se obter uma solução de compromisso. Dentre elas, as mais freqüentes são: algoritmos pseudo-polinomiais, algoritmos probabilísticos, e algoritmos de aproximação.

Nosso interesse concentra-se na área de algoritmos de aproximação. As principais características dos algoritmos de aproximação é ser eficiente (polinomial) e ter garantia de desempenho. Essa garantia é uma medida da proximidade da solução encontrada pelo algoritmo em relação a uma solução ótima do problema. De acordo com a qualidade dessa medida, definem-se várias classes de algoritmos de aproximação. Nessa área, o interesse é projetar algoritmos de aproximação e também encontrar limitantes de aproximabilidade (resultados indicando que não é possível encontrar algoritmos com garantia de desempenho melhor do que certos limitantes, a menos que $\mathrm{P}=\mathrm{NP}$ ). Essa área teve um intenso desenvolvimento nas duas últimas décadas, em particular na década de 90 , quando o famoso Teorema PCP foi provado por Arora et al. [1, 2, 3], fornecendo uma nova caracterização da classe de problemas NP.

Já na década de 60, o conceito de algoritmo de aproximação estava implícito em resultados obtidos por Graham [14] (sobre escalonamento em máquinas paralelas) e Erdős [13] (sobre grafos bipartidos). Mais tarde, Johnson [24] formalizou os conceitos de razão de aproximação e algoritmo de aproximação. Na década de 90 esta área passou a receber um tratamento mais sistemático. Surgiram técnicas aplicáveis a toda uma gama de problemas: métodos baseados em programação linear (método primal, dual e primal-dual), métodos probabilísticos, métodos baseados em programação semidefinida, etc. Na mesma época apareceram resultados de inaproximabilidade, mostrando que certos problemas podem ser aproximados, mas não tão bem como seria desejável (a menos que $\mathrm{P}=\mathrm{NP}$ ). Recentemente, diversos livros têm sido publicados sobre o assunto $[4,20,29]$, mostrando seu reconhecimento como uma disciplina importante. 


\subsection{Empacotamento de subgrafos em grafos}

Um problema clássico da área de otimização combinatória é o problema de encontrar, num dado grafo, um emparelhamento (coleção de arestas disjuntas) máximo. Uma generalização natural deste problema consiste em substituir arestas disjuntas por subgrafos disjuntos, sendo estes pré-estabelecidos, dados através de uma família $\mathcal{F}$ de grafos. Este problema é conhecido como $\mathcal{F}$-empacotamento, definido da seguinte forma. Dado um grafo $G=(V, E)$ encontre um subgrafo $H$ em $G$, tal que $H$ seja uma união de grafos (disjuntos nos vértices ou arestas), cada um dos quais é isomorfo a algum grafo da família $\mathcal{F}$, e tal que $H$ tenha o maior número possível de vértices ou arestas.

Notamos que há várias variantes desse problema: pode-se considerar grafos disjuntos nos vértices ou nas arestas, e a função objetivo pode ser maximizar o número de vértices ou o número de arestas que pertencem a $H$ (no caso mais geral, pode-se ter pesos associados aos vértices e/ou às arestas). Não existem muitas famílias $\mathcal{F}$ para as quais as propriedades de aproximabilidade e/ou inaproximabilidade dos correspondentes problemas de $\mathcal{F}$-empacotamento são completamente entendidas. A maioria dos resultados obtidos trata da complexidade computacional desses problemas.

Surpreendentemente, o problema do $\mathcal{F}$-empacotamento pode ser resolvido em tempo polinomial para algumas famílias não-triviais $\mathcal{F}$, e muitos resultados importantes sobre emparelhamentos podem ser generalizados para esses casos. Listamos, em seguida, algumas das famílias $\mathcal{F}$ estudadas até agora para o problema de encontrar um subgrafo $H$ que seja uma união de grafos disjuntos nos vértices e que tenha o maior número possivel de vértices.

- $\mathcal{F}$ consiste de um único grafo com pelo menos 3 vértices em alguma componente conexo: o problema é NP-difícil. Este resultado segue do resultado provado em [18].

- $\mathcal{F}$ consiste de uma aresta e um conjunto de grafos hipo-emparelháveis (hypomatchable): o problema está em $\mathrm{P}[19,12]$. (Um grafo $G$ é hipo-emparelhável se $G-v$ tem um emparelhamento perfeito para todo $v \in V_{G}$.)

- $\mathcal{F}$ consiste de estrelas $S_{i}, 1 \leq i \leq r$, onde $r$ é um inteiro fixo: está em P [17].

Questões relativas à aproximabilidade de problemas de $\mathcal{F}$-empacotamento (que são NPdifíceis) foram investigadas para algumas poucas famílias $\mathcal{F}$. Dentre essas famílias, destaca-se o caso em que $\mathcal{F}$ consiste de um único triângulo (uma clique de tamanho 3). Este caso, aparentemente simples, é um problema clássico que tem sido objeto de alguns estudos, mas que ainda carece de resultados mais satisfatórios. Por exemplo, para grafos arbitrários, seria interessante responder a questão da existência ou não de algoritmos com garantia de aproximação melhor do que as já conhecidas; para certas classes especiais de grafos, exibir algoritmos polinomiais, ou melhorar razões conhecidas e provar limitantes de aproximação. Este problema e um outro correlato são os assuntos centrais da nossa pesquisa. Mencionamos a seguir esses problemas.

Empacotamento Máximo de Triângulos Disjuntos nos Vértices (VTP): dado um grafo simples $G$, encontrar uma coleção máxima de triângulos em $G$ dois a dois disjuntos nos vértices. 
Empacotamento Máximo de Triângulos Disjuntos nas Arestas (ETP): dado um grafo simples $G$, encontrar uma coleção máxima de triângulos em $G$ dois a dois disjuntos nas arestas.

$\mathrm{K}_{2}+\mathrm{K}_{3}$-empacotamento: é o problema do $\mathcal{F}$-empacotamento no caso em que $\mathcal{F}=\left\{K_{2}, K_{3}\right\}$, e o objetivo é encontrar $H \subseteq G$ que seja uma união de grafos disjuntos nos vértices, cada um dos quais é isomorfo a um grafo de $\mathcal{F}$, e tal que $H$ tenha o maior número possível de arestas.

O problema VTP surge em problemas de escalonamento e problemas de 3-agrupamentos de pessoas: dado um conjunto de pessoas e afinidades entre elas, encontrar grupos disjuntos de 3 membros cada, de modo que as pessoas num mesmo grupo sejam compatíveis e o número de pessoas isoladas seja mínimo [11]. O problema ETP tem aplicações em biologia computacional [7]. Os problemas VTP e ETP são NP-difíceis [25, 21].

Um dos resultados mais relevantes para os problemas VTP e ETP foram obtidos por Hurkens e Schrijver [23] em 1989. O resultado geral de Hurkens e Schrijver [23] sobre empacotamento máximo de $k$-conjuntos implica que um algoritmo simples de busca local é uma $\left(\frac{3}{2}+\varepsilon\right)$-aproximação para o VTP e o ETP. Esta é a melhor razão de aproximação que se conhece para os dois problemas (para grafos arbitrários).

Para um dado inteiro $k \geq 3$, denotamos por VTP- $k$ (resp. ETP- $k$ ) o problema VTP (resp. ETP) restrito a grafos com grau máximo $k$. Em 2002, Caprara e Rizzi [7] exibiram algoritmos polinomiais para os problemas VTP-3 e ETP-4. Eles mostraram ainda que o problema VTP-4 (veja também [6]) e ETP-5 são APX-difíceis (isto é, não admitem um esquema de aproximação polinomial, a menos que $\mathrm{P}=\mathrm{NP}$ ).

Considerando que, em termos de grau, instâncias com grau máximo 4 para o VTP (resp. 5 para o ETP) constituem um caso difícil que está no limite entre instâncias difíceis e fáceis [7], investimos um tempo no estudo dos problemas VTP-4 e ETP-5, na tentativa de encontrar um resultado positivo de aproximabilidade ou um limitante inferior para a razão de aproximação. Conseguimos [27] melhorar a razão de aproximação para esses casos APX-difíceis: obtivemos um algoritmo de aproximação para o problema VTP-4 cuja razão de aproximação é $3-\frac{\sqrt{13}}{2}+\varepsilon$, e um algoritmo para o ETP-5 com razão de aproximação $\frac{4}{3}$.

Para um dado grafo $G$, denotamos por $\mathcal{T}_{V}(G)$ (resp. $\mathcal{T}_{E}(G)$ ) a coleção dos conjuntos dos vértices (resp. arestas) de todos os triângulos em $G$. É fácil ver que o problema VTP num grafo $G$ pode ser reduzido ao problema de encontrar um subconjunto de vértices independente de cardinalidade máxima (problema MIS) no grafo de interseção de $\mathcal{T}_{V}(G)$. Claramente, esta é uma AP-redução: um algoritmo de $\rho$-aproximação para o problema MIS leva a um algoritmo de $\rho$-aproximação para o VTP. Nosso algoritmo para o problema VTP-4 se baseia nesse fato. Ele aplica no grafo de entrada $G$ algumas reduções, que preservam a razão de aproximação, para obter um grafo $G^{\prime}$ no qual todo triângulo intersecta no máximo 3 outros triângulos. Em seguida, no grafo de interseção de $\mathcal{T}_{V}\left(G^{\prime}\right)$, aplica o algoritmo de $\rho(k)$-aproximação de Chlebík e Chlebíková [10], onde $\rho(k)=3-\frac{\sqrt{13}}{2}+\frac{13-\sqrt{13}}{52 k}$ e $k$ é um parâmetro inteiro fixo, para o problema MIS em grafos de grau máximo 3. Observamos que $\rho(4)$ é um pouco menor que 1,25 ; e $\rho(k)$ é um pouco menor que 1,2 para $k>65$.

Já o algoritmo que desenvolvemos para o problema ETP-5 é baseado numa busca local. A idéia desse algoritmo consiste em verificar se existe um certo subgrafo $H$ (chamado grafo 
de Hajós) em G. Se existe, o algoritmo adiciona (de maneira definitiva) alguns triângulos de $H$ ao conjunto $\mathcal{A}^{*}$ a ser devolvido pelo algoritmo, retira $E_{H}$ do grafo $G$ e repete o processo. Caso contrário, ele aplica o algoritmo de busca local de Hurkens e Schrijver mencionado anteriormente.

Obtivemos também um algoritmo linear para o problema VTP em grafos de intervalos unitários. Um grafo de intervalos é um grafo de interseção de um conjunto finito de intervalos em $\mathbb{R}$. Quando todos esses intervalos são de mesmo tamanho, um tal grafo é chamado grafo de intervalos unitários. Nosso algoritmo é guloso e faz uso da caracterização de tais grafos obtida por Looges e Olariu [26]: um grafo $G=(V, E)$ é um grafo de intervalos unitários se e somente se existe uma ordem linear $<$ dos vértices de $G$ tal que, para cada escolha de vértices $u, v, w$ temos que "se $u<v<w$ e $u w \in E$ então $u v, v w \in E$."

$\mathrm{O}$ problema do $\mathrm{K}_{2}+\mathrm{K}_{3}$-empacotamento, na versão aqui considerada (maximizar o número de arestas de $H$ ), tem sido pouco investigada. Encontramos apenas um artigo de Halldórsson [16] sobre cobertura de $k$-conjuntos que fornece uma 3/2-aproximação para esse problema. Observamos que quando o objetivo é maximizar o número de vértices de $H$ então o problema correspondente pode ser resolvido em tempo polinomial [19]. Já o problema de nosso interesse é NP-difícil.

Provamos que o problema do $K_{2}+K_{3}$-empacotamento é APX-difícil para grafos com grau máximo 5. Para isso, mostramos uma L-redução do problema VTP restrito a grafos de grau máximo 4. Mostramos também que o $\mathrm{K}_{2}+\mathrm{K}_{3}$-empacotamento é APX-difícil mesmo para grafos de grau máximo 4 e onde toda aresta pertence a algum triângulo. A L-redução é do problema MAX2SAT3. Ela é mais elaborada, e é baseada na idéia de Caprara e Rizzi [7] para mostrar que o problema VTP-4 é APX-completo.

Além disso, mostramos uma $\left(1+\frac{1}{3} \rho\right)$-aproximação para o $\mathrm{K}_{2}+\mathrm{K}_{3}$-empacotamento, onde $\rho$ é uma razão de aproximação para o problema VTP. Nosso algoritmo inicialmente usa uma rotina para encontrar um número máximo de triângulos disjuntos nos vértices, e depois complementa a solução com um emparelhamento máximo no grafo obtido removendo-se os vértices dos triângulos que a rotina encontrou. Usando como rotina o algoritmo de Hurkens e Schrijver [23] para o VTP, temos que a razão de aproximação do nosso algoritmo é $\frac{3}{2}+\varepsilon$. Ressaltamos ainda que o algoritmo de aproximação para o VTP-4 que obtivemos (com razão um pouco menor que 1,2$)$ resulta em um algoritmo de aproximação com razão 1,4 para o problema do $K_{3}+K_{2}$-empacotamento em grafos de grau máximo 4 .

Apresentamos aqui uma $3 / 2$-aproximação para o problema do $K_{2}+K_{3}$-empacotamento proposta recentemente por Yuster. 


\section{Capítulo 2}

\section{Preliminares}

Neste capítulo introduzimos alguns conceitos e estabelecemos a notação que utilizamos nos próximos capítulos.

\subsection{Teoria dos Grafos}

Um grafo $G$ é um par $\left(V_{G}, E_{G}\right)$ de conjuntos finitos, onde $E_{G}$ é um conjunto de pares nãoordenados de elementos distintos de $V_{G}$. Quando $G$ estiver subentendido, omitimos o índice ${ }_{G}$. Por exemplo, em vez de $V_{G}$ e $E_{G}$, escrevemos simplesmente $V$ e $E$. Os elementos de $V_{G}$ são chamados vértices, e os elementos de $E_{G}$ de arestas. Por brevidade, denotamos uma aresta $\{u, v\}$ por $u v$ ou $v u$. Se $\alpha=u v$, dizemos que $\alpha$ incide em $u$ e em $v$ e que $u$ e $v$ são os extremos de $\alpha$. Também dizemos que $u$ e $v$ são vizinhos ou adjacentes e que $u$ e $v$ são ligados por $\alpha$. Duas arestas distintas são ditas adjacentes se elas têm um extremo em comum. Observamos que o objeto que definimos como grafo é normalmente chamado de grafo simples.

Um grafo $G$ é vazio se $V_{G}=E_{G}=\emptyset$. A ordem de um grafo $G$, denotada por $n_{G}$, é o número de seus vértices. O grau de um vértice $v$ de um grafo $G$ é o número de arestas incidentes em $v$ e será denotado por $d_{G}(v)$. Um vértice de um grafo é isolado se seu grau é zero. O grau máximo é o número $\Delta(G):=\max \left\{d_{G}(v): v \in V_{G}\right\}$.

Um conjunto de vértices ou arestas dois a dois não-adjacentes é chamado independente. No caso de vértices, um conjunto independente é também chamado estável. Um conjunto independente de arestas de um grafo é chamado de emparelhamento.

Se todos os vértices de um grafo $G$ de ordem $n$ são dois a dois adjacentes dizemos que $G$ é completo e o denotamos por $K_{n}$; o grafo $K_{3}$ é chamado triângulo.

Um grafo $H$ é um subgrafo de um grafo $G$ se $V_{H} \subseteq V_{G}$ e $E_{H} \subseteq E_{G}$; escrevemos $H \subseteq G$. A união de dois grafos $G$ e $H$, denotada por $G \cup H$, é o grafo $\left(V_{G} \cup V_{H}, E_{G} \cup E_{H}\right)$. A interseção de dois grafos $G$ e $H$, denotada por $G \cap H$, é o grafo $\left(V_{G} \cap V_{H}, E_{G} \cap E_{H}\right)$. Dois grafos $G$ e $H$ se intersectam se $G \cap H$ é um grafo não-vazio.

Se $U$ é um subconjunto de $V_{G}$, a vizinhança de $U$, denotada por $\Gamma_{G}(U)$, é o conjunto dos vértices de $V_{G} \backslash U$ adjacentes a pelo menos um vértice de $U$.

Se $U \subseteq V_{G}$, então o subgrafo de $G$ induzido por $U$, denotado por $G[U]$, é o subgrafo de $G$ cujo conjunto de vértices é $U$ e cujo conjunto de arestas é o conjunto das arestas de $G$ que têm ambos os extremos em $U$. Denotamos por $G-U$ o grafo $G\left[V_{G} \backslash U\right]$; para o caso em que 
$U=\{v\}$, abreviamos $G-\{v\}$ por $G-v$. Similarmente para $U \subseteq E_{G}$.

Um grafo é bipartido se $V_{G}$ admite uma partição em duas classes de modo que cada aresta tem extremos em classes distintas; uma tal partição é chamada bipartição do grafo. Um grafo bipartido completo é um grafo bipartido no qual todo par de vértices de classes distintas é uma aresta. Denotamos por $K_{m, n}$ o grafo completo com bipartição $\left(V_{1}, V_{2}\right)$ tal que $\left|V_{1}\right|=m$ e $\left|V_{2}\right|=n$.

Um caminho é um grafo $P$ com $V_{P}=\left\{v_{1}, \ldots, v_{k}\right\}$ não-vazio e $E_{P}=\left\{v_{1} v_{2}, v_{2} v_{3}, \ldots, v_{k-1} v_{k}\right\}$. Podemos representar um tal caminho por $v_{1} v_{2} \cdots v_{k}$. Se $P=v_{1} v_{2} \cdots v_{k}$ é um caminho com $k \geq 3$, então $\left(V_{P}, E_{P} \cup\left\{v_{k} v_{1}\right\}\right)$ é chamado de circuito. Uma corda de um circuito é uma aresta que liga dois vértices não consecutivos deste circuito. O comprimento de um caminho é o número de suas arestas; definimos analogamente o comprimento de um circuito.

Um grafo é conexo se, para todo par de vértices distintos $u$ e $v$, contém um caminho com extremos $u$ e $v$. Os subgrafos conexos maximais de um grafo são chamados componentes. Uma clique em um grafo é um conjunto de vértices dois a dois adjacentes.

O grafo de interseção de uma coleção de conjuntos $\mathcal{T}$ é o grafo $H$ com $V_{H}:=\mathcal{T}$ e $X Y \in$ $E_{H} \Leftrightarrow X \cap Y \neq \emptyset$. Um grafo de intervalos é um grafo de interseção de um conjunto finito de intervalos em $\mathbb{R}$. Quando todos esses intervalos são de mesmo tamanho, um tal grafo é chamado grafo de intervalos unitários (ou grafo indiferença). Um grafo é cordal se todo circuito de tamanho maior que 3 tem uma corda. Um grafo é livre de $k$-garras ( $k$-claw free) se ele não contém nenhum subgrafo completo bipartido $K_{1, k}$ como subgrafo induzido. Um cografo é um grafo que não tem caminho de comprimento 4 induzido. Um grafo é split se o seu conjunto de vértices pode ser particionado em um conjunto independente e uma clique.

Para um dado número real $\lambda>0$, denote por $C_{\lambda}$ um conjunto finito de círculos de mesmo raio no plano, onde a distância entre os centros de quaisquer dois círculos é pelo menos $\lambda$. Um grafo disco-unitário de $\lambda$-precisão é um grafo cujos vértices correspondem aos círculos de algum conjunto $C_{\lambda}$ (como definimos), dois de seus vértices são adjacentes se e somente se os círculos correspondentes se intersectam

O problema do Conjunto Independente Máximo (MIS) é definido como segue. Dado um grafo $G$, encontre um subconjunto de vértices independente de cardinalidade máxima em $G$.

\subsection{Conjuntos e problemas de empacotamento}

Para uma dada família $\mathcal{F}$ de grafos o problema do $\mathcal{F}$-empacotamento é definido da seguinte forma. Dado um grafo $G$, encontre um subgrafo $H$ em $G$, tal que $H$ seja uma união de grafos (disjuntos nos vértices ou arestas), cada um dos quais é isomorfo a algum grafo da família $\mathcal{F}$, e tal que $H$ tenha o maior número possível de vértices ou arestas.

Estudaremos o problema do $\mathcal{F}$-empacotamento no caso em que $\mathcal{F}=\left\{K_{3}\right\}$, e o objetivo é encontrar um grafo $H \subseteq G$ que seja uma união de grafos disjuntos nos vértices (resp. arestas), sendo cada um deles isomorfo a $K_{3}$, tal que $H$ tenha o maior número possível de arestas. Esses problemas também são conhecidos pela seguinte denominação:

Problema do Empacotamento Máximo de Triângulos Disjuntos nos Vértices (VTP): dado um grafo $G$, encontrar uma coleção máxima de triângulos em $G$ dois a dois disjuntos nos vértices. Problema do Empacotamento Máximo de Triângulos Disjuntos nas Arestas (ETP): dado um grafo $G$, encontrar uma coleção máxima de triângulos em $G$ dois a dois disjuntos nas 
arestas. Para um dado grafo $G$, denotamos por $\mathcal{T}_{V}(G)$ (resp. $\mathcal{T}_{E}(G)$ ) a coleção dos conjuntos dos vértices (resp. arestas) de todos os triângulos em $G$. Para simplificar, vamos nos referir a uma coleção de triângulos disjuntos nos vértices (resp. arestas) de $G$ como um empacotamento de $\mathcal{T}_{V}(G)$ (resp. $\mathcal{T}_{E}(G)$ ). Para um dado inteiro $k \geq 3$, denotamos por VTP- $k$ (resp. ETP- $k$ ) o problema VTP (resp. ETP) restrito a grafos com grau máximo $k$.

Uma redução natural que podemos fazer nas instâncias dos problemas VTP e ETP consiste em retirar os vértices isolados e as arestas que não pertencem a nenhum triângulo. Portanto, no estudo dos problemas de empacotamento de triângulos, restringimos nossa atenção a grafos nos quais toda aresta pertence a algum triângulo; tais grafos serão chamados irredundantes.

Denotamos por $\mathcal{T}_{G}$ a coleção de todos os triângulos em $G$. Um triângulo cujos vértices são $u, v$ e $w$ é denotado por $[u, v, w]$. O grau de $T \in \mathcal{T}_{G}$, denotado por $d_{G}(T)$, é o número de triângulos em $G$ diferentes de $T$ que intersectam $T$.

Um empacotamento de $\left\{K_{2}, K_{3}\right\}$ em um grafo $G$ é um subgrafo $H$ em $G$ tal que $H$ é uma união de grafos disjuntos nos vértices, cada um dos quais é isomorfo a $K_{2}$ ou $K_{3}$. Se $\mathcal{A}$ é um empacotamento de $\left\{K_{2}, K_{3}\right\}$ em um grafo $G$, então o valor de $\mathcal{A}$ é o número de arestas de $G$ que $\mathcal{A}$ cobre, e o conjunto $\mathcal{T}_{\mathcal{A}}$ (resp. $\mathcal{E}_{\mathcal{A}}$ ) é a coleção de todos os triângulos (resp. arestas) em $\mathcal{A}$. O problema do $K_{2}+K_{3}$-empacotamento é o problema de encontrar num grafo $G$ um empacotamento de $\left\{K_{2}, K_{3}\right\}$ de valor máximo.

Um $k$-conjunto é um conjunto com exatamente $k$ elementos. Dada uma coleção $\mathcal{F}$ de conjuntos, dizemos que uma subcoleção $\mathcal{T}$ de $\mathcal{F}$ cobre um conjunto finito $E$ se todo elemento de $E$ pertence a algum conjunto de $\mathcal{T}$. Neste caso, dizemos também que $\mathcal{T}$ é uma cobertura de $E$. O problema da cobertura mínima por $k$-conjuntos $(k$-SC) consiste no seguinte: dados um conjunto finito $E$ e uma coleção finita $\mathcal{F}$ de subconjuntos de $E$ tamanho no máximo $k$, encontrar uma cobertura $\mathcal{T}$ de $E$ de cardinalidade mínima.

Seja $V$ um conjunto finito de elementos e $E_{1}, \ldots, E_{l} \subseteq V$. Dizemos que a coleção $E_{1}, \ldots, E_{l}$ tem um sistema de representantes distintos (SRD) se existem elementos $e_{1}, \ldots, e_{l}$ dois a dois distintos tais que $e_{i} \in E_{i}$, para todo $i=1, \ldots, l$. Uma coleção $E_{1}, \ldots, E_{l}$ tem um $t$-SRD se qualquer coleção de no máximo $t$ conjuntos entre $E_{1}, \ldots, E_{l}$ tem um SRD. Note que pelo teorema de Hall, as seguintes duas afirmações são equivalentes:

- Uma coleção $E_{1}, \ldots, E_{l}$ tem um $t$-SRD

- Para todo $p \leq t$, quaisquer $p$ conjuntos entre $E_{1}, \ldots, E_{l}$ cobrem pelo menos $p$ elementos de $V$.

\subsection{Classes de problemas, algoritmos e complexidade}

Um algoritmo de $\rho$-aproximação para um problema de maximização $\Pi$ é um algoritmo polinomial tel que, para toda instância $I$ do problema, devolve uma solução $S$ cujo valor, $\operatorname{val}_{\Pi}(I, S)$, é pelo menos $\frac{1}{\rho} \operatorname{opt}_{\Pi}(I)$, onde $\operatorname{opt}_{\Pi}(I)$ é o valor de uma solução ótima de $I$. (o parâmetro $\rho$ pode ser um número maior ou igual a 1 , ou uma função do tamanho da instância). Se um tal algoritmo existe, $\Pi$ pertence à classe de problemas APX. Dizemos que $\rho$ é uma razão de aproximação ou garantia do algoritmo. Um esquema de aproximação polinomial (PTAS) para $\Pi$ é uma família de algoritmos $\left\{A_{\varepsilon}: \varepsilon \in(0,1)\right\}$ tal que para cada $\varepsilon, A_{\varepsilon}$ é um algoritmo de $\frac{1}{1-\varepsilon}$-aproximação para П. 
Denotamos por $\operatorname{Sol}(I)$ o conjunto das soluções viáveis de uma instância $I$. Uma APredução de um problema de otimização $\Pi_{1}$ para um problema de otimização $\Pi_{2}$ é um terno $(f, g, \beta)$ em que $f$ e $g$ são algoritmos e $\beta$ é um número racional positivo tais que:

- $f$ recebe um número racional positivo $\delta$ e uma instância $I$ de $\Pi_{1}$, e devolve uma instância $f(\delta, I)$ de $\Pi_{2}$,

- g recebe um número racional positivo $\delta$, uma instância $I$ de $\Pi_{1}$ e um elemento $S_{2}$ em $\operatorname{Sol}(f(\delta, I))$, e devolve $g\left(\delta, I, S_{2}\right)$ em $\operatorname{Sol}(I)$,

- para todo número racional positivo $\delta$, os algoritmos $f(\delta, \cdot)$ e $g(\delta, \cdot, \cdot)$ são polinomiais,

- para toda instância $I$ de $\Pi_{1}$, todo número racional positivo $\delta$, e todo $S_{2} \operatorname{em} \operatorname{Sol}(f(\delta, I))$, vale que se

$$
\begin{aligned}
& (1-\delta) \operatorname{opt}(f(\delta, I)) \leq \operatorname{val}\left(f(\delta, I), S_{2}\right) \leq(1+\delta) \operatorname{opt}(f(\delta, I)), \\
& \text { então }(1-\beta \delta) \operatorname{opt}(I) \leq \operatorname{val}\left(I, g\left(\delta, I, S_{2}\right)\right) \leq(1+\beta \delta) \operatorname{opt}(I) .
\end{aligned}
$$

Se existe uma AP-redução de um problema de otimização $\Pi_{1}$ a um problema de otimização $\Pi_{2}$, então dizemos que $\Pi_{1}$ é AP-redutível a $\Pi_{2}$. Um problema de otimização $\Pi$ é APX-difícil se todo problema em APX é AP-redutível a $\Pi$, e APX-completo se é APX-difícil e está em APX.

Uma L-redução de um problema de otimização $\Pi_{1}$ para um problema de otimização $\Pi_{2}$ é uma quádrupla $(f, g, \alpha, \beta)$ que consiste de dois algoritmos polinomiais $f$ e $g$ e duas constantes positivas $\alpha$ e $\beta$ satisfazendo as seguintes condições:

- para toda instância $I_{1}$ de $\Pi_{1}$, temos que $f\left(I_{1}\right)$ é uma instância de $\Pi_{2}$,

- dada uma instância $I_{1}$ de $\Pi_{1}$ e qualquer solução viável $S$ de $f\left(I_{1}\right)$, temos que $g\left(I_{1}, S\right)$ é uma solução viável para a instância $I_{1}$ de $\Pi_{1}$,

- $\left|\operatorname{opt}_{\Pi_{2}}\left(f\left(I_{1}\right)\right)\right| \leq \alpha\left|\operatorname{opt}_{\Pi_{1}}\left(I_{1}\right)\right|$, para toda instância $I_{1}$ de $\Pi_{1}$,

- $\left|\operatorname{opt}_{\Pi_{1}}\left(I_{1}\right)-\operatorname{val}_{\Pi_{1}}\left(I_{1}, g\left(I_{1}, S\right)\right)\right| \leq \beta\left|\operatorname{opt}_{\Pi_{2}}\left(f\left(I_{1}\right)\right)-\operatorname{val}_{\Pi_{2}}\left(f\left(I_{1}\right), S\right)\right|$, para toda instância $I_{1}$ de $\Pi_{1}$ e toda solução viável $S$ de $f\left(I_{1}\right)$.

Usamos a notação $\Pi_{1} \leq_{L} \Pi_{2}$ para indicar que existe uma L-redução de $\Pi_{1}$ para $\Pi_{2}$. Se $\Pi_{1} \leq_{L} \Pi_{2}$ e $\Pi_{2} \in \mathrm{APX}$, então $\Pi_{1} \in \mathrm{APX}$. Mais precisamente, a existência de uma $\left(\frac{1}{1-\varepsilon}\right)$ aproximação para $\Pi_{2}$ implica a existência de uma $\left(\frac{1}{1-\alpha \beta \varepsilon}\right)$-aproximação para $\Pi_{1}$. Em se tratando de problemas em APX, a existência de uma L-redução implica na existência de uma AP-redução. Mais precisamente, se $\Pi_{1} \leq_{L} \Pi_{2}$ e $\Pi_{1}$ está em APX, então $\Pi_{1}$ é AP-redutível a $\Pi_{2}$. Assim, para mostrar que um problema $\Pi_{2}$ é APX-difícil basta mostrar que existe um problema APX-completo $\Pi_{1}$ tal que $\Pi_{1} \leq_{L} \Pi_{2}$ (veja em [4]). 


\section{Capítulo 3}

\section{Empacotamento de triângulos disjuntos nos vértices}

\subsection{Introdução}

O problema de Empacotamento Máximo de Triângulos Disjuntos nos Vértices (VTP) é NPdifícil [25, 21]. Temos interesse em obter algoritmos de aproximação para esse problema, resultados de inaproximabilidade, ou obter classes/famílias de instâncias para as quais este problema é polinomial.

Hurkens e Schrijver [23] apresentaram um algoritmo para o problema do empacotamento máximo de $k$-conjuntos, $k \geq 2$ : dada uma família $\mathcal{F}$ de $k$-conjuntos, encontre um empacotamento máximo de $\mathcal{F}$. Trata-se de um algoritmo simples de busca local. No caso particular em que $k=3$, tal algoritmo é uma $\left(\frac{3}{2}+\varepsilon\right)$-aproximação para o VTP. A razão de aproximação obtida por Hurkens e Schrijver é a melhor razão de aproximação que se conhece para o VTP (para grafos arbitrários).

Em 1994, Baker [5] provou que o problema VTP restrito a grafos planares admite um esquema de aproximação polinomial (PTAS). Conforme [7], este resultado pode ser estendido para o ETP. Os dois problemas admitem tal esquema também para grafos disco-unitários de $\lambda$-precisão [22]. O problema VTP é NP-difícil mesmo quando restrito a grafos cordais e é polinomial para as classes de grafos split e cografos [15].

Para um dado inteiro $k \geq 3$, denotamos por VTP- $k$ (resp. ETP- $k$ ) o problema VTP (resp. ETP) restrito a grafos com grau máximo $k$. Em 2002, Caprara e Rizzi [7] mostraram que o VTP-3 está em P, e que o VTP-4 (veja também [6]) é APX-difícil, isto é, não admite um esquema de aproximação polinomial a menos que $\mathrm{P}=$ NP. Chlebík e Chlebíková [9] mostraram que 95/94 é um limitante inferior para a razão de aproximação para o problema VTP-4.

Apresentamos agora um resultado simples que usaremos na análise da a complexidade de nossos algoritmos.

Lema 3.1 número de triângulos em um grafo $G$ de grau máximo $\Delta$ é no máximo $\frac{\Delta(\Delta-1) n_{G}}{6}$.

Demonstração. Claramente, toda aresta de $G$ pertence a no máximo $\Delta-1$ triângulos. Então $|E|(\Delta-1)$ contabiliza o número máximo possível de triângulos em $G$, onde cada triângulo é 
contado 3 vezes (uma vez para cada uma de suas arestas). Como $|E| \leq \frac{\Delta n_{G}}{2}$, temos que

$$
\left|\mathcal{T}_{G}\right| \leq \frac{|E|(\Delta-1)}{3} \leq \frac{\Delta(\Delta-1) n_{G}}{6} .
$$

Neste capítulo apresentamos inicialmente o resultado obtido por Hurkens e Schrijver [23] em 1989 para o problema VTP. Em seguida, apresentamos um algoritmo de aproximação para o VTP-4 cuja razão de aproximação é $3-\frac{\sqrt{13}}{2}+\varepsilon$, e um algoritmo exato para o VTP em grafos de intervalos unitários.

Neste capítulo restringimos nossa atenção aos grafos irredundantes. Quando escrevemos $G-U$ (para $U \subseteq V_{G}$ ou $U \subseteq E_{G}$ ), estamos supondo que os vértices isolados e as arestas que não pertencem a nenhum triângulo foram removidos também.

\subsection{Grafos arbitrários}

Observamos que um algoritmo guloso simples que retorna uma coleção maximal de triângulos disjuntos nos vértices é uma 3-aproximação para o problema VTP. Isto porque todo triângulo da uma solução obtida pelo algoritmo guloso intersecta no máximo 3 triângulos de uma solução ótima.

\subsubsection{Algoritmo de Hurkens e Schrijver}

Um dos resultados mais relevantes para o problema VTP segue de um resultado mais geral (sobre empacotamento máximo de $k$-conjuntos), obtido por Hurkens e Schrijver [23] em 1989. Descrevemos a seguir esse resultado. Dados um número inteiro fixo $t$ e uma coleção $\mathcal{T}$ de $k$-conjuntos, considere o seguinte algoritmo.

Algoritmo $\operatorname{HS}(\mathcal{T}, t)$ :

Dada uma coleção $\mathcal{C} \subseteq \mathcal{T}$ já construída, de conjuntos dois a dois disjuntos, verifique se existem $p \leq t$ conjuntos dois a dois disjuntos em $\mathcal{T} \backslash \mathcal{C}$ que intersectam no máximo $p-1$ conjuntos da coleção $\mathcal{C}$. Se existir, troque os conjuntos, obtendo uma coleção maior $\mathcal{C}$, e repita o processo. Caso contrário, devolva $\mathcal{C}$ (que chamamos de $t$-ótimo). A troca acima consiste em incluir em $\mathcal{C}$ os novos $p$ conjuntos de $\mathcal{T} \backslash \mathcal{C}$ e remover no máximo $p-1$ conjuntos que os intersectam.

Note que o algoritmo $\operatorname{HS}(\mathcal{T}, t)$ se baseia numa busca local e encontra uma coleção de conjuntos em $\mathcal{T}$ dois a dois disjuntos. Claramente, este algoritmo resolve o VTP. Basta tomar $k=3$ e $\mathcal{T}=\mathcal{T}_{V}(G)$, onde $G$ é o grafo de entrada. Lembramos que $\mathcal{T}_{V}(G)$ denota a coleção dos conjuntos dos vértices de todos os triângulos em $G$. O resultado de Hurkens e Schrijver [23] sobre empacotamento máximo de $k$-conjuntos implica que o algoritmo $\operatorname{HS}(\mathcal{T}, t)$ é uma $\left(\frac{3}{2}+\varepsilon\right)$ aproximação para o VTP, onde $\varepsilon$ é inversamente proporcional a $t$ (conforme $t$ cresce, $\varepsilon$ tende a zero). Eles exibiram ainda, para cada $t$, instâncias para as quais o algoritmo acima produz soluções que atingem tal razão em relação ao empacotamento máximo de triângulos disjuntos 
nos vértices. A razão de aproximação obtida por Hurkens e Schrijver é a melhor razão de aproximação que se conhece para o VTP (para grafos arbitrários).

Apresentamos em seguida o resultado geral de Hurkens e Schrijver [23] sobre empacotamento máximo de conjuntos, derivando dai o resultado para o problema VTP.

Teorema 3.2 Seja $Y$ um conjunto com $k$ elementos e $E_{1}, E_{2}, \ldots, E_{l}$ subconjuntos de $Y$. Se todo elemento de $Y$ está contido em no máximo 3 dos conjuntos $E_{1}, E_{2}, \ldots, E_{l}$, e qualquer coleção de no máximo $t$ conjuntos entre $E_{1}, E_{2}, \ldots, E_{l}$ tem um $\mathrm{SRD}$, então

$$
\begin{aligned}
\frac{l}{k} & \leq \frac{3 \cdot 2^{r}-3}{2 \cdot 2^{r}-3}, \text { se } t=2 r-1 e \\
\frac{l}{k} & \leq \frac{3 \cdot 2^{r}-2}{2 \cdot 2^{r}-2}, \text { se } t=2 r .
\end{aligned}
$$

Lembramos que a coleção $E_{1}, \ldots, E_{l}$ tem um sistema de representantes distintos se existem elementos $e_{1}, \ldots, e_{l}$ dois a dois distintos tais que $e_{i} \in E_{i}$, para todo $i=1, \ldots, l$. Para provar este teorema, ordenamos os conjuntos $E_{1}, \ldots, E_{l}$ de modo que

$$
\begin{aligned}
\left|E_{1}\right| & =\cdots=\left|E_{q}\right| \geq 3 \\
\left|E_{q+1}\right| & =\cdots=\left|E_{h}\right|=2 \\
\left|E_{h+1}\right| & =\cdots=\left|E_{l}\right|=1 .
\end{aligned}
$$

Definimos ainda o conjunto $W:=\bigcup_{i=h+1}^{l} E_{i}$. Sejam $T_{1}, \ldots, T_{k}$ os elementos de $Y$. Para cada $i=1, \ldots, q$ seja $X_{i}$ um conjunto de tamanho $\left|E_{i}\right|-2$, disjunto de $E_{1} \cup \cdots \cup E_{l}$, e tal que $X_{i} \cap X_{j}=\emptyset$, se $i \neq j$. Seja $X_{1} \cup \cdots \cup X_{q}=\left\{y_{1}, \ldots, y_{s}\right\}$. Considere agora a coleção de conjuntos

$$
\left(E_{1} \backslash W\right) \cup X_{1}, \ldots,\left(E_{q} \backslash W\right) \cup X_{q}, E_{q+1} \backslash W, \ldots, E_{h} \backslash W,\left\{y_{1}\right\}, \ldots,\left\{y_{s}\right\} .
$$

Para a prova do Teorema 3.2 precisaremos do seguinte lema.

Lema 3.3 Para todo $t \geq 3$, se $E_{1}, \ldots, E_{l}$ tem um $t$-SRD, então a coleção 3.3 tem um $(t-2)$ SRD.

Demonstração. Suponha que a coleção (3.3) não tenha um $(t-2)$-SRD. Portanto, existe uma coleção $\Pi$ de conjuntos de (3.3) tal que $|\Pi|=p, p \leq t-2$ e $|\cup \Pi| \leq p-1$. Escolha $\Pi$ de forma que $p$ seja mínimo. Então,

$$
|\cup \Pi|=p-1 .
$$

De fato, suponha que $|\Pi|=p$ e $|\cup \Pi| \leq p-2$, e considere a coleção $\Pi^{\prime}:=\Pi \backslash\{S\}$, onde $S$ é qualquer elemento de $\Pi$. Temos que $p^{\prime}:=\left|\Pi^{\prime}\right|=p-1$ e $\left|\cup \Pi^{\prime}\right| \leq p-2=p^{\prime}-1$. Porém $p^{\prime}<p$, o que contradiz a minimalidade de $p$.

Ainda, temos que

cada elemento de $\cup \Pi$ é coberto por pelo menos dois conjuntos em $\Pi$.

De fato, suponha que exista um elemento $T$ de $\cup \Pi$ tal que $T$ seja coberto por exatamente um conjunto $S$ em $\Pi$. Considere $\Pi^{\prime}:=\Pi \backslash\{S\}$. Como $T$ é coberto somente por $S$, temos que 
$\cup \Pi^{\prime} \subseteq \cup \Pi \backslash\{T\}$. Usando (3.4) temos que $p^{\prime}:=\left|\Pi^{\prime}\right|=p-1$ e $\left|\cup \Pi^{\prime}\right| \leq|\cup \Pi|-1=p-2=p^{\prime}-1$, o que contradiz a minimalidade de $p$.

Mostramos em seguida que de (3.5) segue a seguinte afirmação.

$$
\text { Para cada } i=1, \ldots, q \text { e } x \in X_{i} \text {, temos que }\{x\} \in \Pi \Leftrightarrow\left(E_{i} \backslash W\right) \cup X_{i} \in \Pi \text {. }
$$

Suponha que $\{x\} \in \Pi$. Claramente, $x \in \cup \Pi$. De (3.5) temos que $x$ pertence a mais algum conjunto em П. Como todo $X_{i}$ é disjunto de $E_{1} \cup \cdots \cup E_{l}$ e $X_{i} \cap X_{j}=\emptyset$ para $i \neq j$, temos que os únicos conjuntos da família (3.3) que contêm $x$ são $\{x\}$ e $\left(E_{i} \backslash W\right) \cup X_{i}$. Logo, $\left(E_{i} \backslash W\right) \cup X_{i} \in \Pi$. Analogamente, $\left(E_{i} \backslash W\right) \cup X_{i} \in \Pi$ implica que $\{x\} \in \Pi$.

Podemos supor, sem perda de generalidade, que

todos os conjuntos $\left(E_{1} \backslash W\right) \cup X_{1}, \ldots,\left(E_{q} \backslash W\right) \cup X_{q}, E_{q+1} \backslash W, \ldots, E_{h} \backslash W$ pertencem a $\Pi$.

De fato, suponha que exista $j, 1 \leq j \leq h$, tal que $\left(E_{j} \backslash W\right) \cup X_{j} \notin \Pi$ (ou $E_{j} \backslash W \notin \Pi$ ). Considere a família $\mathcal{E}^{\prime}:=\left\{E_{1}, \ldots, E_{l}\right\} \backslash\left\{E_{j}\right\}$. Como $E_{1}, \ldots, E_{l}$ tem um $t$-SRD, sabemos que quaisquer $s(s \leq t)$ conjuntos entre $E_{1}, \ldots, E_{l}$ cobrem pelo menos $s$ elementos de $V$. Considere $s$ conjuntos em $\mathcal{E}^{\prime}(s \leq t)$. Estes conjuntos pertencem à coleção $E_{1}, \ldots, E_{l}$, portanto cobrem pelo menos $s$ elementos de $V$. Ademais, como $\Pi$ não foi mudado, temos que $|\Pi|=p$ e $|\cup \Pi| \leq p-1$. Logo, podemos remover $E_{j}$ de $E_{1}, \ldots, E_{l}$ se $\left(E_{j} \backslash W\right) \cup X_{j} \notin \Pi$ (ou $E_{j} \backslash W \notin \Pi$ ).

Note que

$$
\text { os conjuntos } E_{h+1}, \ldots, E_{l} \text { são dois a dois disjuntos. }
$$

Suponha a existência de dois conjuntos $E_{i_{1}}, E_{i_{2}}, h+1 \leq i_{1}<i_{2} \leq l$, tais que $E_{i_{1}}=E_{i_{2}}$. Como $\left|E_{i_{1}}\right|=\left|E_{i_{2}}\right|=1$, concluímos que a coleção $E_{1}, \ldots, E_{l}$ não tem um $t$-SRD (pois $t \geq 3$ ), o que é uma contradição.

Ainda, sem perda de generalidade, podemos supor que

$$
\left(E_{1} \cup \cdots \cup E_{h}\right) \cap W=E_{h+1} \cup \cdots \cup E_{l} .
$$

De fato, como $W=\bigcup_{i=h+1}^{l} E_{i}$, temos que $\left(E_{1} \cup \cdots \cup E_{h}\right) \cap W \subseteq E_{h+1} \cup \cdots \cup E_{l}$. Suponha agora que $E_{h+1} \cup \cdots \cup E_{l} \nsubseteq\left(E_{1} \cup \cdots \cup E_{h}\right) \cap W$. Então, existe $T_{j}, 1 \leq j \leq k$, tal que $T_{j} \in E_{h+1} \cup \cdots \cup E_{l}=W$ e $T_{j} \notin\left(E_{1} \cup \cdots \cup E_{h}\right) \cap W$, ou seja, $T_{j} \notin E_{1} \cup \cdots \cup E_{h}$. De (3.8) temos que existe somente um conjunto $E_{i}, h+1 \leq i \leq l$, tal que $\left\{T_{j}\right\}=E_{i}$. Ainda, como $T_{j} \notin E_{1} \cup \cdots \cup E_{h}$, temos que $T_{j}$ pertence a somente um conjunto de $\Pi$, o que contradiz (3.5).

Note que

$$
s=\left|X_{1} \cup \cdots \cup X_{q}\right|=\sum_{i=1}^{h}\left(\left|E_{i}\right|-2\right) .
$$

Além disso,

$$
p=h+s .
$$

De fato, por (3.7), todos os conjuntos $\left(E_{1} \backslash W\right) \cup X_{1}, \ldots,\left(E_{q} \backslash W\right) \cup X_{q}, E_{q+1} \backslash W, \ldots, E_{h} \backslash W$ pertencem a П. Logo, por (3.6) concluímos que $\{x\} \in \Pi$ para todo $x \in X_{i}$ e $i=1, \ldots, q$. Segue imediatamente que $\Pi$ é toda a coleção (3.3), ou seja, $|\Pi|=p=h+s$. 
Do fato de que П é toda a coleção (3.3), e das afirmações (3.4) e (3.11) segue que

$$
\left|\bigcup_{i=1}^{h}\left(E_{i} \backslash W\right)\right|=|\cup \Pi|-s=(p-1)-s=(p-s)-1=h-1 .
$$

Agora, de (3.9) temos que

$$
\left|\bigcup_{i=1}^{l}\left(E_{i} \cap W\right)\right|=\left|\bigcup_{i=1}^{h}\left(E_{i} \cap W\right)\right|=|W| .
$$

Ainda, de (3.8) segue que

$$
|W|=l-h .
$$

Usando (3.12), (3.13) e (3.14), temos que

$$
\begin{aligned}
\left|\bigcup_{i=1}^{l} E_{i}\right| & =\left|\bigcup_{i=1}^{h}\left(E_{i} \backslash W\right)\right|+\left|\bigcup_{i=1}^{l}\left(E_{i} \cap W\right)\right| \\
& =\left|\bigcup_{i=1}^{h}\left(E_{i} \backslash W\right)\right|+\left|\bigcup_{i=1}^{h}\left(E_{i} \cap W\right)\right| \\
& =(h-1)+|W|=h-1+(l-h)=l-1 .
\end{aligned}
$$

Ainda, por (3.5), temos que $\sum_{i=1}^{h}\left|E_{i} \backslash W\right| \geq 2\left|\bigcup_{i=1}^{h}\left(E_{i} \backslash W\right)\right|$. Usando esta inequação, o fato de que $p \leq t-2$, e as afirmações (3.14), (3.9), (3.12), (3.10) e (3.11), temos que

$$
\begin{aligned}
l & =h+(l-h) \\
& =h+\left|E_{h+1} \cup \cdots \cup E_{l}\right| \\
& =h+\left|\left(E_{1} \cup \cdots E_{h}\right) \cap W\right| \\
& =h+\left|\bigcup_{i=1}^{h}\left(E_{i} \cap W\right)\right| \leq h+\sum_{i=1}^{h}\left|E_{i} \cap W\right|=h+\sum_{i=1}^{h}\left(\left|E_{i}\right|-\left|E_{i} \backslash W\right|\right) \\
& =h+\sum_{i=1}^{h}\left|E_{i}\right|-\sum_{i=1}^{h}\left|E_{i} \backslash W\right| \leq h+\sum_{i=1}^{h}\left|E_{i}\right|-2\left|\bigcup_{i=1}^{h}\left(E_{i} \backslash W\right)\right| \\
& =h+2 h+\sum_{i=1}^{h}\left|E_{i}-2\right|-2(h-1) \\
& =h+2 h+s-2 h+2 \\
& =h+s+2=p+2 \leq t .
\end{aligned}
$$

Então, de (3.15) e (3.16) temos que $\left|\cup_{i=1}^{l} E_{i}\right|=l-1$ e $l \leq t$, o que contradiz o fato de que a coleção $E_{1}, \ldots, E_{l}$ tem um $t$-SRD.

Apresentamos a seguir a prova do Teorema 3.2.

Demonstração. (do Teorema 3.2) A prova é por indução em $t$. Denotamos por $\varphi(t) o$ termo do lado direito das inequações em (3.1). Note que, como todo elemento de $Y$ está 
contido em no máximo três conjuntos de $E_{1}, \ldots, E_{l}$, temos que

$$
\sum_{i=1}^{l}\left|E_{i}\right| \leq 3 k \text {. }
$$

Seja $t=1$. Como $E_{1}, \ldots, E_{l}$ tem um $t$-SRD, temos que todo $E_{i}, i=1, \ldots, l$ é não-vazio. Logo, $l \leq \sum_{i=1}^{l}\left|E_{i}\right|$. De (3.17) temos que $l \leq 3 k$, ou seja, $l / k \leq 3=\varphi(1)$.

Seja $t=2$. Logo, qualquer coleção de no máximo 2 conjuntos entre $E_{1}, \ldots, E_{l}$ tem um SRD. Portanto, $E_{i} \neq \emptyset$, para $i=1, \ldots, l$. Ainda, a união de quaisquer 2 conjuntos entre $E_{1}, \ldots, E_{l}$ cobrem pelo menos 2 elementos de $Y$. Portanto, não existem 2 conjuntos $E_{i_{1}}, E_{i_{2}}$, $h+1 \leq i_{1}<i_{2} \leq i_{2} \leq l$, tais que $E_{i_{1}}=E_{i_{2}}$. Logo,

$$
l-h \leq k \text {. }
$$

Agora, de (3.2), (3.14) e (3.17) temos que

$$
l+h=2 h+(l-h) \leq \sum_{i=1}^{h}\left|E_{i}\right|+\sum_{i=h+1}^{l}\left|E_{i}\right|=\sum_{i=1}^{l}\left|E_{i}\right| \leq 3 k .
$$

Usando a inequação acima e (3.18), obtemos $2 l=(l-h)+(l+h) \leq k+3 k=4 k$. Logo, $l / k \leq 2=\varphi(2)$.

Seja agora $t \geq 3$. Considere a coleção (3.3) de conjuntos sobre

$$
Y^{\prime}:=\bigcup_{i=1}^{l^{\prime}} E_{i}^{\prime}
$$

Note que

$$
l^{\prime}=h+s \text { e } k^{\prime}:=\left|Y^{\prime}\right|=|Y|-|W|+s=k-|W|+s .
$$

Pelo Lema 3.3 , temos que $E_{1}^{\prime}, \ldots, E_{l^{\prime}}^{\prime}$ tem um $(t-2)$-SRD. Claramente, todo elemento de $Y^{\prime}$ está em no máximo 3 dos conjuntos $E_{1}^{\prime}, \ldots, E_{l^{\prime}}^{\prime}$. Logo, pela hipótese de indução,

$$
l^{\prime} \leq \varphi(t-2) k^{\prime} .
$$

Usando a inequação acima e (3.19), concluímos que $h+s \leq \varphi(t-2)(k-|W|+s)$. Reescrevendo essa última desigualdade obtemos

$$
\varphi(t-2)|W|+h-(\varphi(t-2)-1) s \leq \varphi(t-2) k .
$$

Agora, de (3.10) e (3.17) temos que

$$
|W|+2 h+s=|W|+2 h+\sum_{i=1}^{h}\left(\left|E_{i}\right|-2\right)=|W|+\sum_{i=1}^{h}\left|E_{i}\right|=\sum_{i=1}^{l}\left|E_{i}\right| \leq 3 k .
$$

Usando (3.14), temos que

$$
\begin{aligned}
l & =(l-h)+h=|W|+h \\
& =\frac{|W|(2 \varphi(t-2)-1)}{2 \varphi(t-2)-1}+\frac{h(2 \varphi(t-2)-1)}{2 \varphi(t-2)-1}=\frac{2|W| \varphi(t-2)-|W|+2 h \varphi(t-2)-h}{2 \varphi(t-2)-1} \quad \text { (3.22) } \\
& =\frac{\varphi(t-2)|W|+h-\varphi(t-2) s+s+\varphi(t-2)|W|-|W|+2 h \varphi(t-2)-2 h+s \varphi(t-2)-s}{2 \varphi(t-2)-1} \\
& =\frac{1}{2 \varphi(t-2)-1}(\varphi(t-2)|W|+h-(\varphi(t-2)-1) s)+\frac{\varphi(t-2)-1}{2 \varphi(t-2)-1}(|W|+2 h+s) .
\end{aligned}
$$


Agora, (3.20), (3.21) e (3.22) implicam que

$$
l \leq \frac{1}{2 \varphi(t-2)-1} \varphi(t-2) k+\frac{\varphi(t-2)-1}{2 \varphi(t-2)-1} 3 k=k \frac{4 \varphi(t-2)-3}{2 \varphi(t-2)-1} .
$$

Logo,

$$
\frac{l}{k} \leq \frac{4 \varphi(t-2)-3}{2 \varphi(t-2)-1}
$$

Para $t=2 r-1$, usando (3.23) e a hipótese de indução, temos que

$$
\frac{l}{k} \leq \frac{4 \frac{3 \cdot 2^{r-1}-3}{2 \cdot 2^{r-1}-3}-3}{2 \frac{3 \cdot 2^{r-1}-3}{2 \cdot 2^{r-1}-3}-1}=\frac{3 \cdot 2^{r}-3}{2 \cdot 2^{r}-3}=\varphi(t) .
$$

Para $t=2 r$, usando (3.23) e a hipótese de indução, temos que

$$
\frac{l}{k} \leq \frac{4 \frac{3 \cdot 2^{r-1}-2}{2 \cdot 2^{r-1}-2}-3}{2 \frac{3 \cdot 2^{r-1}-2}{2 \cdot 2^{r-1}-2}-1}=\frac{3 \cdot 2^{r}-2}{2 \cdot 2^{r}-2}=\varphi(t) .
$$

Corolário 3.4 Seja $G=(V, E)$ um grafo, $\mathcal{T}_{V}(G)$ a coleção dos conjuntos dos vértices de todos os triângulos em $G$, e $t$ um inteiro positivo. O algoritmo $\operatorname{HS}\left(\mathcal{T}_{V}(G), t\right)$ é uma $\left(\frac{3}{2}+\varepsilon\right)$ aproximação para o problema $\mathrm{VTP}$, onde $\varepsilon$ é inversamente proporcional a $t$.

Demonstração. Sejam $T_{1}, \ldots, T_{k}$ os triângulos devolvidos pelo algoritmo $\operatorname{HS}\left(\mathcal{T}_{V}(G), t\right)$, e $T_{1}^{\prime}, \ldots, T_{l}^{\prime}$ um empacotamento máximo de $\mathcal{T}_{V}(G)$. Definimos $Y:=\left\{T_{1}, \ldots, T_{k}\right\}$. Para cada $i=1, \ldots, l$, definimos o conjunto $E_{i}:=\left\{T_{j} \mid T_{j} \cap T_{i}^{\prime} \neq \emptyset\right\}$. Observe que todo $T_{j}, j=1, \ldots, k$, está contido em no máximo três conjuntos de $E_{1}, \ldots, E_{l}$, pois os triângulos $T_{1}^{\prime}, \ldots, T_{l}^{\prime}$ são dois a dois disjuntos nos vértices. Ainda, como a solução do algoritmo $\operatorname{HS}\left(\mathcal{T}_{V}(G), t\right)$ é $t$-ótima, temos que a união de quaisquer $p, p \leq t$ triângulos de $T_{1}^{\prime}, \ldots, T_{l}^{\prime}$ intersecta pelo menos $p$ triângulos de $T_{1}, \ldots, T_{k}$. Portanto, qualquer coleção de no máximo $t$ conjuntos entre $E_{1}, \ldots, E_{l}$ tem um SRD. Pelo Teorema 3.2 temos que

$$
\begin{aligned}
\frac{l}{k} & \leq \frac{3 \cdot 2^{r}-3}{2 \cdot 2^{r}-3}, \text { se } t=2 r-1 \\
\frac{l}{k} & \leq \frac{3 \cdot 2^{r}-2}{2 \cdot 2^{r}-2}, \text { se } t=2 r
\end{aligned}
$$

\subsection{Empacotamento de triângulos disjuntos nos vértices em grafos com grau máximo 4}

\subsubsection{Introdução}

Nesta seção restringimos nossa atenção aos grafos irredundantes de grau máximo 4 . 
Considerando que, em termos de grau, instâncias com grau máximo 4 para o problema VTP constituem um caso difícil que está no limite entre instâncias difíceis e fáceis [7], investimos um tempo no estudo do problema VTP-4, procurando obter um algoritmo com uma razão de aproximação melhor. Desenvolvemos um algoritmo de aproximação para o problema VTP-4 cuja razão de aproximação é $3-\frac{\sqrt{13}}{2}+\varepsilon$.

É fácil ver que o problema VTP num grafo $G$ pode ser reduzido ao problema de encontrar um subconjunto de vértices independentes de cardinalidade máxima (problema MIS) no grafo de interseção de $\mathcal{T}_{V}(G)$. Claramente, esta é uma AP-redução: um algoritmo de $\rho$-aproximação para o problema MIS leva a um algoritmo de $\rho$-aproximação para o VTP.

Nesta seção descrevemos um algoritmo de aproximação, chamado de VT4 $4_{k}$, para o problema VTP em grafos irredundantes de grau máximo 4, onde $k$ é um parâmetro inteiro fixo. Este algoritmo aplica no grafo de entrada $G$ algumas reduções que preservam a razão de aproximação, e obtém um grafo $G^{\prime}$ no qual todo triângulo intersecta no máximo 3 outros triângulos. Em seguida, no grafo de interseção de $\mathcal{T}_{V}\left(G^{\prime}\right)$, aplica o algoritmo $\mathrm{MIS}_{k}$ para o problema MIS em grafos de grau máximo 3. O algoritmo MIS3 ${ }_{k}$, obtido por Chlebík e Chlebíková [10], é uma $\rho(k)$-aproximação onde $\rho(k)=3-\frac{\sqrt{13}}{2}+\frac{13-\sqrt{13}}{52 k}$. Observamos que $\rho(4)$ é um pouco menor que 1,25 ; e $\rho(k)$ é um pouco menor que 1,2 para $k>65$. Vale notar que o algoritmo $\mathrm{MIS}_{k}$ é essencialmente o algoritmo de Berman e Fujito [6], mas a análise cuidadosa feita por Chlebík e Chlebíková em [10] melhora a razão $\left(\frac{6}{5}+\frac{1}{5 k}\right)$ obtida em [6].

$\mathrm{O}$ algoritmo $\mathrm{MIS}_{k}$ aplica inicialmente reduções ao grafo de entrada $G^{\prime}$ que preservam a razão de aproximação, entre outros: Branchy Reduction - se existe um caminho $v_{1} v_{2} v_{3} v_{4}$ onde $v_{2}$ e $v_{3}$ têm grau 2, remove $v_{2}$ e $v_{3}$ do grafo e insere a aresta $v_{1} v_{4}$, e Small Commitment Reduction - procura repetidamente um subconjunto $I$ de $V_{G^{\prime}}$ tal que $|I| \leq k$ e $I$ é um conjunto independente máximo no subgrafo induzido pelos vértices em $I$ e seus vizinhos. Se encontrado, $I$ é adicionado ao conjunto que o algoritmo $\mathrm{MIS}_{k}$ devolverá e os vértices em $I$ e seus vizinhos são removidos do grafo $G^{\prime}$.

$\mathrm{O}$ algoritmo $\mathrm{MIS}_{k}$ é baseado em aumento local em duas direções. Um p-aumento local de um conjunto independente $I$ adiciona $p$ vértices e retira $p-1$ vértices de $I$, de modo que o conjunto obtido é independente. Um conjunto independente é $p$-ótimo se ele não admite um $p$-aumento local.

Se $I$ é um conjunto independente no grafo de entrada $G^{\prime}$, o algoritmo MIS3 ${ }_{k}$ iterativamente aplica $O\left(k \log \left|V_{G^{\prime}}\right|\right)$-aumentos em $I$. Quando $I$ se torna $O\left(k \log \left|V_{G^{\prime}}\right|\right)$-ótimo, o algoritmo encontra um conjunto independente máximo $I^{\prime}$ no grafo induzido por $V_{G^{\prime}} \backslash I$ (como o grau máximo de $G^{\prime}$ é 3 , todo vértice no grafo $G^{\prime}\left[V_{G^{\prime}} \backslash I\right]$ tem grau no máximo 2, e portanto $I^{\prime}$ é fácil de encontrar). Se $\left|I^{\prime}\right|>|I|$, o processo se repete com $\left|I^{\prime}\right|$ no lugar de $|I|$. Caso contrário, o algoritmo devolve $I$.

O consumo de tempo para encontrar aumentos locais é $O\left(\left|V_{G^{\prime}}\right|^{1+3 k \log 3}\right)$. Como tais aumentos são feitos no máximo $\left|V_{G^{\prime}}\right|$ vezes, o tempo de execução do algoritmo $\operatorname{MIS} 3_{k}\left(G^{\prime}\right)$ é $O\left(\left|V_{G^{\prime}}\right|^{2+3 k \log 3}\right)$.

Em todas as figuras desta seção, cada vértice quadrado é um vértice comum a dois triângulos de $G$ cuja união é uma borboleta. Um vértice $x$ que é representado por um círculo é saturado, ou seja, nenhuma outra aresta é incidente a $x$, além das que já estão na figura.

Introduzimos agora alguns conceitos que utilizamos neste capítulo. Se dois triângulos $T_{1}$ e $T_{2}$ de $G$ têm um único vértice em comum e não existe outro triângulo em $G$ que intersecta 
ambos $T_{1}$ e $T_{2}$, dizemos que o subgrafo $T_{1} \cup T_{2}$ é uma borboleta em $G$ e denotamos por $v_{T_{1} T_{2}}$ o único vértice comum a $T_{1}$ e $T_{2}$. Uma coleção $\mathcal{T}$ de triângulos disjuntos nos vértices em $G$ é localmente ótima em $G$ se $\left\{V_{T}: T \in \mathcal{T}\right\}$ é um empacotamento máximo da coleção $\left\{V_{T}: T \in \mathcal{T}_{G}, T\right.$ intersecta um triângulo em $\left.\mathcal{T}\right\}$. Quando $\mathcal{T}=\{T\}$, dizemos simplesmente que o triângulo $T$ é localmente ótimo.

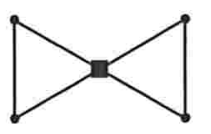

(a)

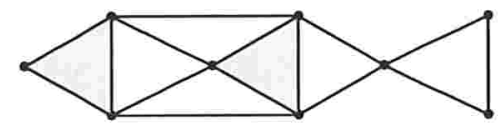

(b)

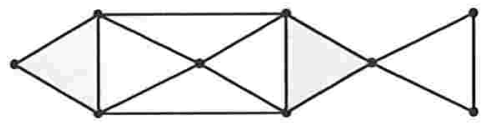

(c)

Figura 3.1: (a) Uma borboleta. (b) Uma coleção localmente ótima. (c) Uma coleção não localmente ótima.

\subsubsection{Algoritmo de aproximação $\mathrm{VT}_{k}$}

Antes de darmos uma descrição formal do algoritmo $\mathrm{VT} 4_{k}$, apresentamos as idéias básicas desse algoritmo. Em cada iteração do algoritmo $\mathrm{VT}_{4}$, uma coleção $\mathcal{T} \subseteq \mathcal{T}_{G},|\mathcal{T}| \leq 2$, localmente ótima em $G$ é repetidamente adicionada a $\mathcal{A}^{*}$ (solução que o algoritmo devolve) e $G$ é atualizado. Se $G$ tem um triângulo $T$ de grau maior que 3, o algoritmo encontra um certo subgrafo $H$ que contém $T$ e aplica uma redução apropriada. A definição de borboleta é crucial pois a redução é baseada no número de triângulos em $H$ que formam uma borboleta com um triângulo fora de $H$ (este número é no máximo 2 , como veremos na seção 3.3.3). Também, vamos provar que, para uma coleção $\mathcal{T}$ localmente ótima em $G$, a adição de $\mathcal{T}$ à solução atual e a remoção de $G$ dos vértices de todos os triângulos em $\mathcal{T}$ preservam a razão de aproximação do algoritmo $\mathrm{VT} 4_{k}$. Ainda, provaremos que cada redução feita no subgrafo $H$ preserva a razão de aproximação do algoritmo.

Descrevemos agora o algoritmo $\mathrm{VT}_{k}$ em mais detalhes. Em cada iteração do algoritmo $\mathrm{VT}_{k}$, uma coleção $\mathcal{T} \subseteq \mathcal{T}_{G},|\mathcal{T}| \leq 2$, localmente ótima em $G$ é repetidamente adicionada a $\mathcal{A}^{*}$ (e $G$ é atualizado) com o objetivo de eliminar instâncias especiais, ou seja, instâncias que têm uma coleção localmente ótima com no máximo 2 triângulos (algumas dessas instâncias especiais são mostradas na Figura 3.2). Fazendo isto, como vamos ver na seção 3.3.3, restam somente algumas poucas instâncias gerais que têm uma estrutura similar.

Se $G$ ainda contém um triângulo $T$ de grau maior que 3, o algoritmo encontra um subgrafo $H$ definido como um subgrafo maximal conexo irredundante de $G$ que contém $T$ e não contém nenhuma borboleta. Explorando a estrutura do grafo $G$ irredundante de grau máximo 4, e usando o fato de que $G$ não contém nenhuma coleção $|\mathcal{T}|,|\mathcal{T}| \leq 2$, localmente ótima em $G$ (caso contrário, ela seria adicionada à solução do algoritmo na primeira etapa), podemos provar que tal grafo $H$ tem uma estrutura muito específica. Mais precisamente, o número de triângulos $T^{\prime}$ em $\mathcal{T}_{H}$ para os quais existe um triângulo em $\mathcal{T}_{G} \backslash \mathcal{T}_{H}$ que forma uma borboleta com $T^{\prime}$ em $G$ é no máximo 2. Além disso, $H$ é isomorfo a um dos grafos da Figura 3.16. Ressaltamos porém que, o subgrafo $H$ pode ser encontrado sem fazer uma busca exaustiva de subgrafos isomorfos aos da Figura 3.16. 


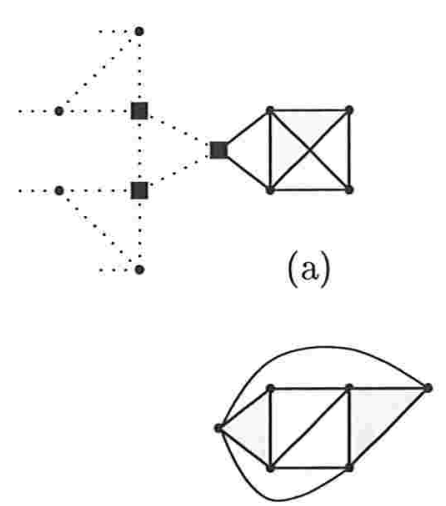

(d)

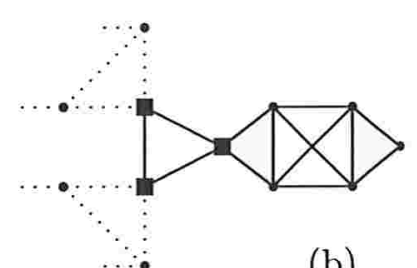

(b)

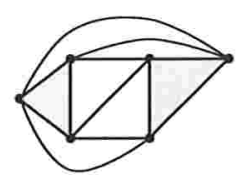

(e)

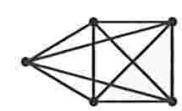

(c)

Figura 3.2: Exemplos das instâncias que têm uma coleção $\mathcal{T}$ localmente ótima com no máximo dois triângulos (os triângulos marcados estão em $\mathcal{T}$, e cada vértice quadrado é comum a dois triângulos em $G$ cuja união é uma borboleta). Os grafos (c), (d) e (e) são componentes de $G$.

Explicamos agora o comportamento do algoritmo em cada possível caso do subgrafo $H$.

Se o subgrafo $H$ tem dois triângulos $T^{\prime}$ e $T^{\prime \prime}$ que formam uma borboleta com um triângulo que não está em $H$, então, como provaremos na seção 3.3.3, $H$ é isomorfo a um dos grafos (a), (b) ou (c) da Figura 3.16. Em todos esses casos o algoritmo executa a redução apropriada. Devido à estrutura especial do grafo $H$, o algoritmo é capaz de decidir rapidamente se existe uma coleção $\mathcal{T} \subseteq \mathcal{T}_{H},|\mathcal{T}|>2$, localmente ótima em $G$. Se tal coleção existe, a redução adiciona $\mathcal{T}$ a $\mathcal{A}^{*}$ e atualiza $G$. Caso contrário, o algoritmo reduz $H$ de modo que no grafo reduzido os triângulos obtidos pela redução têm grau no máximo 3 . O algoritmo decide qual destas reduções aplicar comparando as cardinalidades dos empacotamentos máximos de $\mathcal{T}_{V}(H)$ em casos quando ambos $T^{\prime}$ e $T^{\prime \prime}$ estão no empacotamento, quando exatamente um deles está no empacotamento, e quando nenhum deles está no empacotamento. Para dar uma intuição das reduções, apresentamos alguns exemplos nas Figuras 3.3 e 3.4.

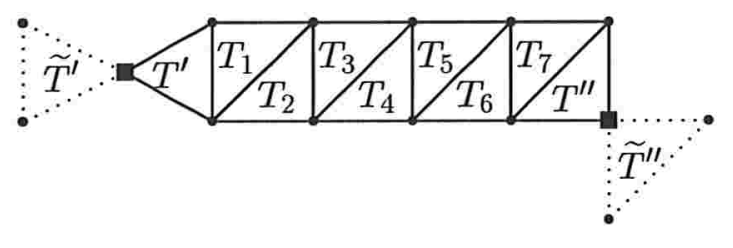

(a)

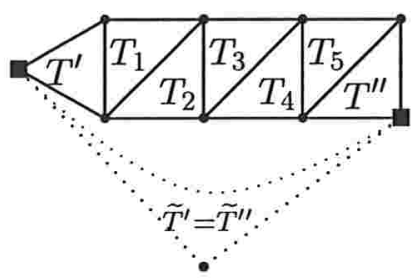

(b)

Figura 3.3: Exemplos do grafo $H$ obtido no algoritmo VT4 $4_{k}$ ( $H$ é o grafo com arestas cheias). $T^{\prime}$ (resp. $T^{\prime \prime}$ ) é um triângulo de $H$ que forma uma borboleta com um triângulo $\widetilde{T}^{\prime}$ (resp. $\widetilde{T}^{\prime \prime}$ ) que não está em $H$. Note que possivelmente $\widetilde{T}^{\prime}=\widetilde{T}^{\prime \prime}$. (a) A coleção $\left\{T_{1}, T_{4}, T_{7}\right\}$ de $\mathcal{T}_{V}(H)$ é localmente ótima em $G$. (b) A coleção $\left\{T^{\prime}, T_{3}, T^{\prime \prime}\right\}$ de $\mathcal{T}_{V}(H)$ é localmente ótima em $G$.

Note que para o exemplo da Figura 3.3(a), os empacotamentos máximos de $\mathcal{T}_{V}(H)$ em todos os casos possíveis (quando ambos $T^{\prime}, T^{\prime \prime}$ estão no empacotamento, quando exatamente um deles está no empacotamento, e quando nenhum deles está no empacotamento) têm a mesma cardinalidade. Observe, ainda, que esses empacotamentos podem ser encontrados 
facilmente. Por exemplo, um empacotamento máximo de $\mathcal{T}_{V}(H)$ no caso em que $T^{\prime}$ está no empacotamento e $T^{\prime \prime}$ não está no empacotamento pode ser obtido tomando-se o triângulo $T^{\prime}$, o triângulo $T_{3}$ (que é localmente ótimo em $H-v_{T^{\prime \prime} \widetilde{T}^{\prime \prime}}-V_{T^{\prime}}$ ) e o triângulo $T_{6}$ (que é localmente ótimo em $\left.H-v_{T^{\prime \prime} \widetilde{T}^{\prime \prime}}-V_{T^{\prime}}-V_{T_{3}}\right)$. Provaremos que neste caso um empacotamento máximo de $\mathcal{T}_{V}(H)$ que não contém $T^{\prime}$ nem $T^{\prime \prime}$ é uma coleção localmente ótima em $G$ (no exemplo, este empacotamento é $\left.\left\{T_{1}, T_{4}, T_{7}\right\}\right)$.

A Figura 3.3(b) mostra um caso para o qual $\widetilde{T}^{\prime}=\widetilde{T}^{\prime \prime}$ e os empacotamentos máximos de $\mathcal{T}_{V}(H)$ não são de mesma cardinalidade nos três seguintes casos: quando ambos $T^{\prime}$ e $T^{\prime \prime}$ estão no empacotamento, quando exatamente um deles está no empacotamento, e quando nenhum deles está no empacotamento. Provaremos que neste caso um empacotamento máximo de $\mathcal{T}_{V}(H)$ que contém ambos $T^{\prime}$ e $T^{\prime \prime}$ é uma coleção localmente ótima em $G$ (no exemplo, este empacotamento é $\left.\left\{T^{\prime}, T_{3}, T^{\prime \prime}\right\}\right)$.

Quanto ao exemplo da Figura 3.4, comparando os empacotamentos máximos de $\mathcal{T}_{V}(H)$ em todos os casos possíveis (quando ambos $T^{\prime}$ e $T^{\prime \prime}$ estão no empacotamento, quando exatamente um deles está no empacotamento, e quando nenhum deles está no empacotamento), notamos o seguinte. Mesmo que os empacotamentos em (c) e (e) tenham a mesma cardinalidade, a solução em (e) é "melhor solução" que a solução em (c) (pois se escolhermos $\left\{T^{\prime}, T_{5}\right\}$ para incluirmos na solução final, precisamos omitir o triângulo $\widetilde{T}^{\prime}$, o que não é o caso se escolhermos $\left\{T_{1}, T_{4}\right\}$ para incluirmos na solução final do algoritmo). Similarmente, mesmo que os empacotamentos em (d) e (e) sejam de mesmo tamanho, incluir $\left\{T_{1}, T_{4}\right\}$ é melhor que incluir $\left\{T_{1}, T^{\prime \prime}\right\}$ na solução final. Portanto, é sempre bom escolher $\left\{T^{\prime}, T_{3}, T^{\prime \prime}\right\}$ ou $\left\{T_{1}, T_{4}\right\}$ (e incluí-los na solução final do algoritmo). Entretanto, não sabemos qual dos dois é melhor globalmente. Portanto, aplicamos Redução $1(H)$, ou seja, substituímos todos os triângulos de $H$, exceto $T^{\prime}$ e $T^{\prime \prime}$, por um novo triângulo $T_{H}$. Aplicamos a redução para eliminar os triângulos de grau maior que 3 , isto é, para podermos aplicar o algoritmo MIS $3_{k}$. Como vamos ver na seção 3.3.3, essa redução preserva a razão de aproximação do algoritmo.

Se o subgrafo $H$ tem somente um triângulo $T^{\prime}$ tal que existe um triângulo em $\mathcal{T}_{G} \backslash \mathcal{T}_{H}$ que forma uma borboleta com $T^{\prime}$ em $G$, então, como vamos provar na seção 3.3.3, $H$ é isomorfo ao grafo da Figura 3.16(d). Além disso, $G\left[V_{H}\right]$ é uma componente de $G$. Observe que para o grafo $G\left[V_{H}\right]$ é fácil encontrar um empacotamento máximo: tome $T^{\prime}, T_{3}, T_{6}, T_{9}$, etc. Note que $T^{\prime}$ é localmente ótimo em $H, T_{3}$ é localmente ótimo em $H-V_{T^{\prime}}, T_{6}$ é localmente ótimo em $H-V_{T^{\prime}}-V_{T_{3}}$, etc.

Se, no entanto, $H$ não tem nenhum triângulo $T^{\prime}$ que forma uma borboleta com um triângulo em $\mathcal{T}_{G} \backslash \mathcal{T}_{H}$, então $H$ é isomorfo ao grafo da Figura 3.16(e). Ademais, todo vértice tem grau 4, e portanto, $G\left[V_{H}\right]$ é uma componente de $G$. Note que se $\widetilde{T}$ é um triângulo em $H$, então $H-V_{\widetilde{T}}$ é isomorfo ao grafo das arestas cheias mostrado na Figura 3.16(d). Mostraremos, portanto, que uma solução ótima do grafo $H$ isomorfo ao grafo da Figura 3.16(e) pode ser obtido tomando-se qualquer triângulo $\widetilde{T}$ de $H$ e uma solução ótima de $H-V_{\widetilde{T}}$. Esta última pode ser construída da mesma forma como fizemos para o grafo da Figura 3.16(d).

Repetimos as iterações enquanto existe um triângulo em $G$ com grau maior que 3. Quando não existe mais tal triângulo, aplicamos o algoritmo $\mathrm{MIS}_{k}$ no grafo de interseção de $\mathcal{T}_{G}$. Finalmente, para cada aplicação (em ordem reversa) de $\operatorname{Redução~}(H)$, fazemos $\operatorname{Restauração~}(H)$, ou seja, adicionamos os triângulos apropriados de $H$ à solução final do algoritmo (veja o exemplo da Figura 3.4). 


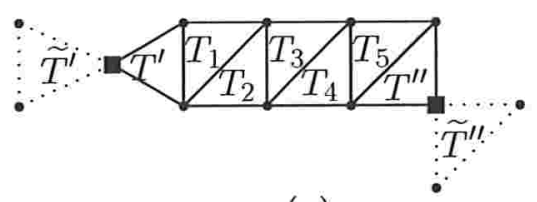

(a)

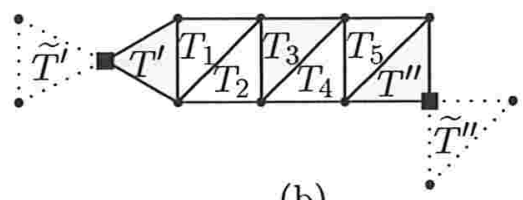

(b)

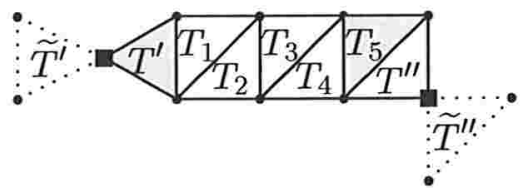

(c)

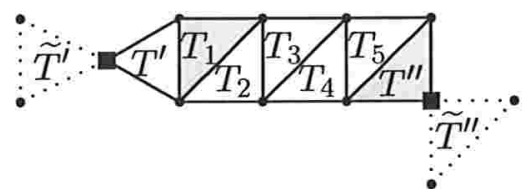

(d)

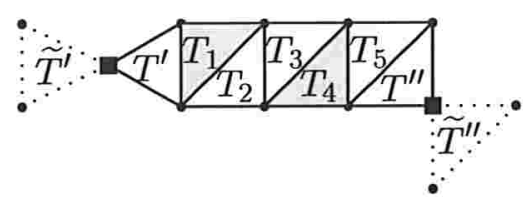

(e)

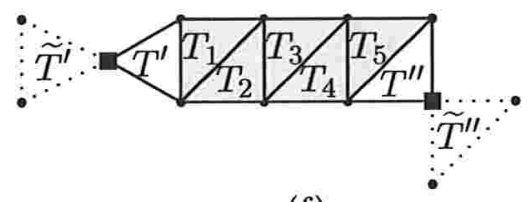

(f)

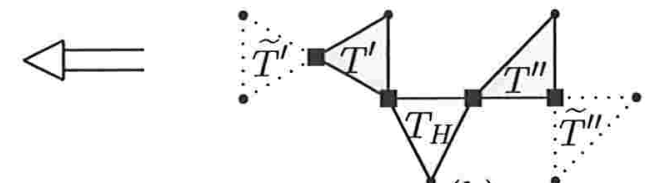

(h)
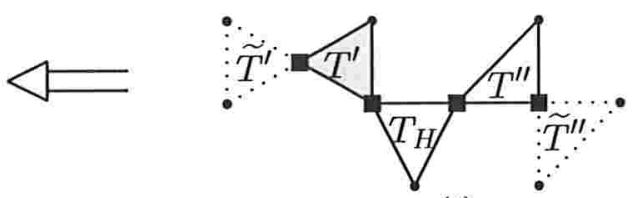

(i)
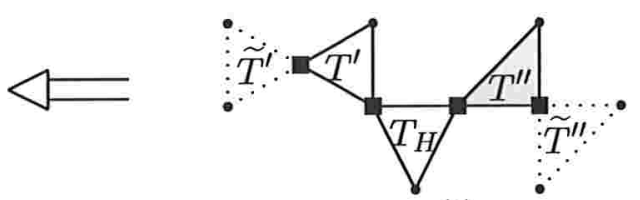

(j)
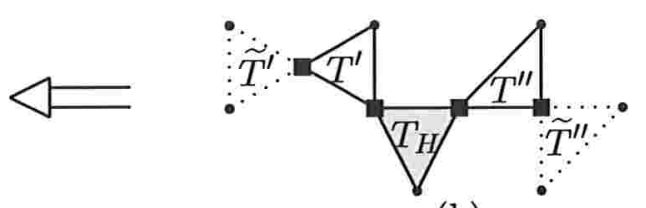

(k)

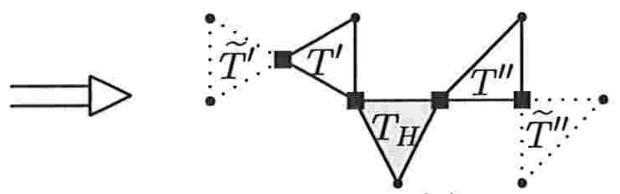

$(\mathrm{g})$

Figura 3.4: (a) Exemplo de um grafo $H$ obtido no algoritmo VT4 $4_{k}$ ( $H$ é o grafo com arestas cheias). $T^{\prime} \cup \widetilde{T}^{\prime}$ e $T^{\prime \prime} \cup \widetilde{T}^{\prime \prime}$ são borboletas em $G$. Dependendo se $T^{\prime}, T^{\prime \prime}$ estão ou não num empacotamento máximo de $\mathcal{T}_{V}(H)$, temos um dos casos (b), (c), (d) ou (e). (b) $\left\{T^{\prime}, T_{3}, T^{\prime \prime}\right\}$ é um empacotamento máximo de $\mathcal{T}_{V}(H)$ que contém $T^{\prime}$ e $T^{\prime \prime}$. (c) $\left\{T^{\prime}, T_{5}\right\}$ é um empacotamento máximo de $\mathcal{T}_{V}(H)$ que contém $T^{\prime}$ mas não $T^{\prime \prime}$. (d) $\left\{T_{1}, T^{\prime \prime}\right\}$ é um empacotamento máximo de $\mathcal{T}_{V}(H)$ que contém $T^{\prime \prime}$ mas não $T^{\prime}$. (e) $\left\{T_{1}, T_{4}\right\}$ é um empacotamento máximo de $\mathcal{T}_{V}(H)$ que não contém $T^{\prime}$ nem $T^{\prime \prime}$. (f) e (g) representam Redução $1(H)$ : ela substitui todos os triângulos de $H$, exceto $T^{\prime}$ e $T^{\prime \prime}$, por um novo triângulo $T_{H}$. (h) Se $T^{\prime}, T^{\prime \prime}$ estão na solução do algoritmo antes da aplicação de Restauração $(H)$, então o procedimento Restauração $(H)$ adiciona $T_{3}$ à solução final. (i) Se $T^{\prime}$ está na solução do algoritmo antes da aplicação de Restauração $(H)$, mas $T^{\prime \prime}$ não, então este procedimento adiciona $T_{5}$ à solução final. (j) Semelhante ao caso (i). (k) Se $T_{H}$ está na solução do algoritmo antes da aplicação de Restauração $(H)$, então o procedimento Restauração $(H)$ remove $T_{H}$ da solução e adiciona $T_{1}$ e $T_{4}$ à solução. 
Apresentamos agora um pseudocódigo do algoritmo $\mathrm{VT}_{k}$.

\section{Algoritmo $\mathrm{VT}_{k}$}

Entrada: Um grafo $G$ irredundante de grau máximo 4 .

$1 \mathcal{A}^{*} \leftarrow \emptyset$

2 ENQUANTO existe um triângulo em $G$ com grau maior que 3

3 FAÇa Enquanto existe $\mathcal{T} \subseteq \mathcal{T}_{G},|\mathcal{T}| \leq 2$, localmente ótimo em $G$

$4 \quad$ FAÇA $\operatorname{Aceita}(\mathcal{T})$

5

6

7

SE existe um triângulo $T \in \mathcal{T}_{G} \operatorname{com} d_{G}(T)>3$

ENTÃo $H \leftarrow$ subgrafo maximal conexo irredundante de $G$ que contém $T$ e não contém nenhuma borboleta

8

9

15 SE $G \neq \emptyset$ ЕNTão $\mathcal{A}^{*} \leftarrow \mathcal{A}^{*} \cup \mathrm{MIS}_{k}$ (grafo de interseção de $\mathcal{T}_{G}$ ) $B_{H} \leftarrow\left\{T^{\prime} \in \mathcal{T}_{H}\right.$ : existe um triângulo em $\mathcal{T}_{G} \backslash \mathcal{T}_{H}$ que forma uma borboleta com $T^{\prime}$ em $\left.G\right\}$

SE $\left|B_{H}\right|$

é igual a 2: Redução $(H)$

é igual a 1: $\operatorname{Sol}_{H} \leftarrow \operatorname{Adota}(H), \mathcal{A}^{*} \leftarrow \mathcal{A}^{*} \cup \operatorname{Sol}_{H}$

é igual a 0 : tome um triângulo $\widetilde{T}$ em $\mathcal{T}_{H}$,

$$
\operatorname{Sol}_{H} \leftarrow\{\widetilde{T}\} \cup \operatorname{Adota}\left(H-V_{\widetilde{T}}\right), \mathcal{A}^{*} \leftarrow \mathcal{A}^{*} \cup \operatorname{Sol}_{H}
$$

16 PARA cada aplicação (em ordem reversa) de Redução $(H)$ FAÇA Restauração $(H)$

17 DEVOLVA $\mathcal{A}^{*}$

Descrevemos em seguida cada procedimento do algoritmo em mais detalhes.

1. Aceita $(\mathcal{T})$ : Adicione $\mathcal{T}$ a $\mathcal{A}^{*}$, e remova os vértices de todos os triângulos em $\mathcal{T}$ do grafo $G$. (O algoritmo executa $\operatorname{Aceita}(\mathcal{T})$ não somente na linha 4, mas também em

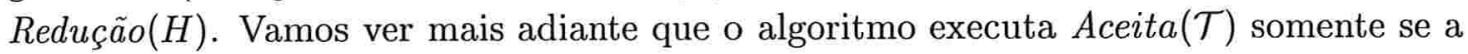
coleção $\mathcal{T}$ é localmente ótima.)

2. $\operatorname{Adota}(H)$ : Faça $\mathcal{E}:=\emptyset$. Enquanto $H \neq \emptyset$, encontre um triângulo $T$ localmente ótimo em $H$, adicione $T$ a $\mathcal{E}$ e remova $V_{T}$ de $H$. Devolva $\mathcal{E}$. (Referimo-nos a $\operatorname{Adota}(H)$ como sendo o conjunto $\mathcal{E}$ devolvido por esse procedimento.)

3. Redução $(H)$ : dado um grafo $H$ como definido no algoritmo, com $\left|B_{H}\right|=2$, este procedimento reduz $H$ de modo que no grafo reduzido os triângulos obtidos pela redução têm grau no máximo 3 (no caso da aplicação de Redução $1(H)$ ou Redução $2(H)$ ), ou adiciona alguns triângulos de $H$ a $\mathcal{A}^{*}$ e atualiza o grafo (isto é, executa $A c e i t a(\mathcal{T})$ ), para um $\left.\mathcal{T} \subseteq \mathcal{T}_{H}\right)$. Mais formalmente, o procedimento Redução $(H)$ é o seguinte.

Sejam $T^{\prime}, T^{\prime \prime} \in B_{H}$ e $\tilde{T}^{\prime}, \widetilde{T}^{\prime \prime} \in \mathcal{T}_{G} \backslash \mathcal{T}_{H}$ tais que $T^{\prime} \cup \widetilde{T}^{\prime}$ e $T^{\prime \prime} \cup \widetilde{T}^{\prime \prime}$ são borboletas em $G$ (possivelmente $\widetilde{T}^{\prime}=\widetilde{T}^{\prime \prime}$ ). Considere os conjuntos:

$$
\begin{aligned}
& \operatorname{Sol}_{T^{\prime} T^{\prime \prime}}:=\left\{T^{\prime}, T^{\prime \prime}\right\} \cup \operatorname{Adota}\left(H-V_{T^{\prime}}-V_{T^{\prime \prime}}\right), \operatorname{Sol}_{T^{\prime} \bar{T}^{\prime \prime}}:=\left\{T^{\prime}\right\} \cup \operatorname{Adota}\left(H-V_{T^{\prime}}-v_{T^{\prime \prime}} \widetilde{T}^{\prime \prime}\right), \\
& \operatorname{Sol}_{\bar{T}^{\prime} T^{\prime \prime}}:=\left\{T^{\prime \prime}\right\} \cup \operatorname{Adota}\left(H-V_{T^{\prime \prime}}-v_{T^{\prime} \widetilde{T}^{\prime}}\right), \quad \operatorname{Sol}_{\bar{T}^{\prime} \bar{T}^{\prime \prime}}:=\operatorname{Adota}\left(H-v_{T^{\prime} \widetilde{T}^{\prime}}-v_{T^{\prime \prime} \widetilde{T}^{\prime \prime}}\right) .
\end{aligned}
$$

Veremos na seção 3.3 .3 que $\operatorname{Sol}_{T^{\prime} T^{\prime \prime}}$ é um empacotamento máximo de $\mathcal{T}_{V}(H)$ que contém $T^{\prime}$ e $T^{\prime \prime}, \operatorname{Sol}_{T^{\prime} \bar{T}^{\prime \prime}}$ é um empacotamento máximo de $\mathcal{T}_{V}(H)$ que contém $T^{\prime}$ mas não $T^{\prime \prime}$, etc. 
(a) Se $\left|\operatorname{Sol}_{T^{\prime} T^{\prime \prime}}\right|=\left|\operatorname{Sol}_{T^{\prime} \bar{T}^{\prime \prime}}\right|=\left|\operatorname{Sol}_{\bar{T}^{\prime} T^{\prime \prime}}\right|=\left|\operatorname{Sol}_{\bar{T}^{\prime} \bar{T}^{\prime \prime}}\right|$ então aplique Aceita $\left(\operatorname{Sol}_{\bar{T}^{\prime} \bar{T}^{\prime \prime}}\right)$. Veja a Figura 3.3(a).

(b) Se a igualdade em (a) não está satisfeita e $\widetilde{T}^{\prime}=\widetilde{T}^{\prime \prime}$ então aplique $\operatorname{Aceita}\left(\operatorname{Sol}_{T^{\prime}} T^{\prime \prime}\right)$. Veja a Figura 3.3(b).

(c) Se $\left|\operatorname{Sol}_{T^{\prime} T^{\prime \prime}}\right|-1=\left|\operatorname{Sol}_{T^{\prime} \bar{T}^{\prime \prime}}\right|=\left|\operatorname{Sol}_{\bar{T}^{\prime} T^{\prime \prime}}\right|=\left|\operatorname{Sol}_{\bar{T}^{\prime} \bar{T}^{\prime \prime}}\right|$ e $\widetilde{T}^{\prime} \neq \widetilde{T}^{\prime \prime}$ então aplique Redução $1(H)$ :

$$
G \leftarrow\left(G-\left(E_{H} \backslash\left\{E_{T^{\prime}} \cup E_{T^{\prime \prime}}\right\}\right)\right) \cup T_{H},
$$

onde $T_{H}:=\left[v^{\prime}, w, v^{\prime \prime}\right], w$ é um vértice novo, $v^{\prime}$ é qualquer vértice de $T^{\prime}$ diferente de $v_{T^{\prime} \widetilde{T}^{\prime}}$, e $v^{\prime \prime}$ é qualquer vértice de $T^{\prime \prime}$ diferente de $v_{T^{\prime \prime} \widetilde{T}^{\prime \prime}}$. Portanto, Redução $1(H)$ substitui todos os triângulos de $H$, exceto $T^{\prime}$ e $T^{\prime \prime}$, por um novo triângulo $T_{H}$ que induz uma borboleta com $T^{\prime}$ e com $T^{\prime \prime}$ no grafo reduzido (veja as Figuras 3.4 (f) e (g)). Provaremos que $T^{\prime}$ e $T^{\prime \prime}$ são disjuntos, ou seja, Redução $1(H)$ está bem definida.

(d) Se $\left|\operatorname{Sol}_{T^{\prime} T^{\prime \prime}}\right|=\left|\operatorname{Sol}_{T^{\prime} \bar{T}^{\prime \prime}}\right|=\left|\operatorname{Sol}_{\bar{T}^{\prime} T^{\prime \prime}}\right|=\left|\operatorname{Sol}_{\bar{T}^{\prime} \bar{T}^{\prime \prime}}\right|+1$ e $\widetilde{T}^{\prime} \neq \tilde{T}^{\prime \prime}$, então aplique Redução $2(H)$ :

$$
G \leftarrow\left(G-E_{H}\right) \cup T_{H}^{1} \cup T_{H}^{2}
$$

onde $T_{H}^{1}:=\left[v_{T^{\prime} \widetilde{T}^{\prime}}, w_{1}, w\right], T_{H}^{2}:=\left[w, w_{2}, v_{T^{\prime \prime} \widetilde{T}^{\prime \prime}}\right]$ e $w_{1}, w, w_{2}$ são vértices novos. Logo, esta redução substitui todos os triângulos de $H$ por dois triângulos novos $T_{H}^{1}$ e $T_{H}^{2}$, tais que $T_{H}^{1}$ induz uma borboleta com $T_{H}^{2}$ e com $\widetilde{T}^{\prime}$; e $T_{H}^{2}$ induz uma borboleta com $\widetilde{T}^{\prime \prime}$ e com $T_{H}^{1}$ no grafo reduzido.

4. Restauração $(H)$ : Se a redução aplicada a $H$ foi Redução $1(H)$ ou Redução $2(H)$, então o procedimento Restauração $(H)$ adiciona os triângulos apropriados de $H$ a $\mathcal{A}^{*}$.

(a) Se a redução aplicada a $H$ foi $\operatorname{Redução~} 1(H)$, então se $T_{H}$ pertence a $\mathcal{A}^{*}$ antes da aplicação de Restauração $(H)$, este procedimento remove $T_{H}$ de $\mathcal{A}^{*}$ e adiciona o conjunto $\operatorname{Sol}_{\bar{T}^{\prime} \bar{T}^{\prime \prime}}$ a ele (este conjunto foi computado no procedimento Redução $(H)$ ); se $T^{\prime}, T^{\prime \prime} \in \mathcal{A}^{*}$, então $\mathcal{A}^{*} \leftarrow \mathcal{A}^{*} \cup \operatorname{Sol}_{T^{\prime} T^{\prime \prime}} ;$ se $T^{\prime} \in \mathcal{A}^{*}, T^{\prime \prime} \notin \mathcal{A}^{*}$, então $\mathcal{A}^{*} \leftarrow$ $\mathcal{A}^{*} \cup \operatorname{Sol}_{T^{\prime} \bar{T}^{\prime \prime}} ;$ e se $T^{\prime} \notin \mathcal{A}^{*}, T^{\prime \prime} \in \mathcal{A}^{*}$, então $\mathcal{A}^{*} \leftarrow \mathcal{A}^{*} \cup \operatorname{Sol}_{\bar{T}^{\prime} T^{\prime \prime}}$ (veja a Figura 3.4).

(b) Se a redução aplicada a $H$ foi $\operatorname{Redução~} 2(H)$, então se $T_{H}^{1}$ pertence a $\mathcal{A}^{*}$ antes da aplicação de Restauração $(H)$, este procedimento adiciona $\operatorname{Sol}_{T^{\prime} \bar{T}^{\prime \prime}}$ a $\mathcal{A}^{*}$ e remove $T_{H}^{1}$; se $T_{H}^{2} \in \mathcal{A}^{*}$, então adiciona $\operatorname{Sol}_{\bar{T}^{\prime} T^{\prime \prime}}$ a $\mathcal{A}^{*}$ e remove $T_{H}^{2} ;$ e se $T_{H}^{1}, T_{H}^{2} \notin \mathcal{A}^{*}$, então adiciona $\operatorname{Sol}_{\bar{T}^{\prime} \bar{T}^{\prime \prime}}$ a $\mathcal{A}^{*}$.

\subsubsection{Desempenho do algoritmo $\mathrm{VT}_{k}$}

Observamos inicialmente o seguinte fato sobre o grafo $H$ definido na linha 6 do algoritmo $\mathrm{VT} 4_{k}$.

Se $H$ não contém borboletas, e $T_{1}, T_{2}$ são triângulos de $H$ que têm somente um vértice $z$ em comum, então existe pelo menos uma aresta de $H$ com um extremo em $V_{T_{1}} \backslash\{z\}$ e outro em $V_{T_{2}} \backslash\{z\}$.

Isso vale pois o grau máximo 4 já foi atingido no vértice $z$. 
Lema 3.5 Para cada iteração do algoritmo VT4 $4_{k}$ para a qual a condição da linha 5 está satisfeita, se $\left|B_{H}\right|=2$, então os triângulos em $B_{H}$ são disjuntos.

Demonstração. Seja $G$ o grafo na linha 5 do algoritmo VT4 $4_{k}, H$ o subgrafo de $G$ (como definido na linha 6) com $\left|B_{H}\right|=2$, e $T^{\prime}, T^{\prime \prime}, \widetilde{T}^{\prime}, \widetilde{T}^{\prime \prime}$ como definidos no procedimento Redução $(H)$.

Suponha que $\left|V_{T^{\prime}} \cap V_{T^{\prime \prime}}\right|=2$, e que $V_{T^{\prime}} \cap V_{T^{\prime \prime}}=\{x, y\}$. Se $\widetilde{T}^{\prime}=\widetilde{T}^{\prime \prime}$, então $T^{\prime} \cup \widetilde{T}^{\prime}$ não é uma borboleta em $G$, uma contradição (veja a Figura $3.5(\mathrm{a})$ ). Logo, $\widetilde{T}^{\prime} \neq \widetilde{T}^{\prime \prime}$. Dos fatos que $v_{T^{\prime} \widetilde{T}^{\prime}}$ e $v_{T^{\prime \prime}} \widetilde{T}^{\prime \prime}$ já têm grau $4, T^{\prime} \cup \widetilde{T}^{\prime}\left(\right.$ resp. $\left.T^{\prime \prime} \cup \widetilde{T}^{\prime \prime}\right)$ é uma borboleta em $G$ e $\Delta(G)=4$, concluímos que existe no máximo mais um triângulo em $G$ que intersecta $x$ ou $y$, a saber, o triângulo $[x, y, z]$. Note que agora $x$ e $y$ têm grau 4 . Como $H$ não contém borboletas, $[x, y, z]$ é o único triângulo de $H$ que contém $z$ (note que $z$ pode ser um vértice na interseção de dois triângulos que formam uma borboleta). Mostramos portanto que $H$ contém $T^{\prime}, T^{\prime \prime}$ e no máximo mais um triângulo $[x, y, z]$ (veja a Figura 3.5(b)). Mas isso implica que $H$ não tem um triângulo $T$ com $d_{G}(T)>3$, uma contradição.

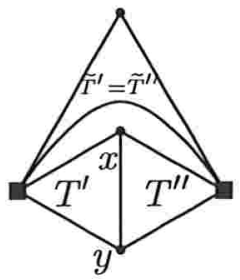

(a)

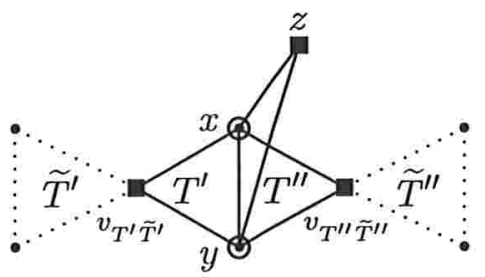

(b)

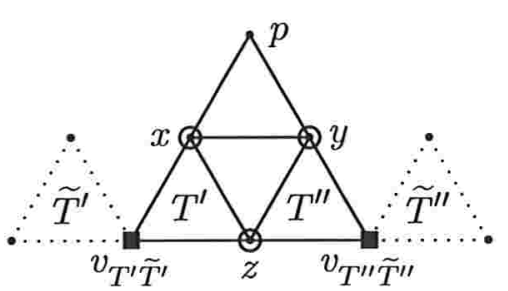

(c)

Figura 3.5: Os triângulos em $B_{H}$ não se intersectam.

Suponha agora que $T^{\prime}$ e $T^{\prime \prime}$ têm somente um vértice em comum. Similarmente como acima, temos que $\widetilde{T}^{\prime} \neq \widetilde{T}^{\prime \prime}$. Se $T^{\prime}=\left\{v_{T^{\prime} \widetilde{T}^{\prime}}, x, z\right\}$ e $T^{\prime \prime}=\left\{v_{T^{\prime \prime} \widetilde{T}^{\prime \prime}}, z, y\right\}$, como $v_{T^{\prime} \widetilde{T}^{\prime}}$ e $v_{T^{\prime \prime}} \widetilde{T}^{\prime \prime}$ já têm grau 4, usando o fato (3.24), temos que $x y \in E_{H}$. Agora, dos fatos que $H$ tem um triângulo $T$ com $d_{G}(T)>3, z$ tem grau 4 e $\Delta(G)=4$, concluímos que existe no máximo um triângulo em $G$ que intersecta $x$ ou $y$, a saber, o triângulo $[x, y, p]$ (veja a Figura 3.5(c)). Mas agora, ambos $x$ e $y$ têm grau 4 e $[x, y, z]$ é localmente ótimo em $G$. Portanto, o algoritmo teria aplicado Aceita $([x, y, z])$ na linha 4 , e novamente temos uma contradição.

Corolário 3.6 O procedimento Redução $1(H)$ está bem definido e não cria novos triângulos em $G$ exceto $T_{H}$.

Demonstração. Segue diretamente do Lema 3.5.

Observamos ainda que Redução $2(H)$ não cria novos triângulos em $G$, exceto $T_{H}^{1}$ e $T_{H}^{2}$, pois $\widetilde{T}^{\prime} \neq \tilde{T}^{\prime \prime}$.

É fácil ver que após a execução de Redução $1(H)$ ou Redução $2(H)$, o grafo obtido ainda tem grau máximo 4 e é irredundante. Mostraremos que todas as outras reduções aplicadas no algoritmo consistem em adicionar a $\mathcal{A}^{*}$ uma coleção $\mathcal{T}$ localmente ótima em $G$ e remover de $G$ os vértices de todos os triângulos em $\mathcal{T}$. Portanto, as propriedades estruturais do grafo de entrada (grau máximo 4 e irredundância) são mantidas em cada iteração. 
Além disso, observamos que

para cada iteração do algoritmo para a qual existe um triângulo em $G$ com grau maior que 3 , onde $G$ é o grafo na linha 5 , não existe nenhuma coleção $\mathcal{T}$, $|\mathcal{T}| \leq 2$, localmente ótima em $G$.

Caso contrário, é claro que o algoritmo aplicaria $\operatorname{Aceita}(\mathcal{T})$ na linha 4.

Lema 3.7 Em cada iteração do algoritmo $\mathrm{VT} 4_{k}$, temos que $d_{G}(x) \geq 3$ para todo $x \in V_{H}$, onde $H$ é o grafo na linha 6 .

Demonstração. Suponha que $H$ tenha um vértice $x \operatorname{com} d_{G}(x)=2$. Seja $T$ o triângulo em $H$ que contém $x$. Se somente um triângulo de $G$ fosse adjacente a $T$, então $T$ seria localmente ótimo em $G$, uma contradição com (3.25). Portanto, $d_{G}(T) \geq 2$. Se existem dois triâgulos $T_{1}$ e $T_{2}$ disjuntos nos vértices que intersectam $T$, então $T \cup T_{1}$ e $T \cup T_{2}$ são borboletas em $G$, ou seja, $H=T$, o que é impossível, pois $H$ tem um triângulo com grau maior que $3 \mathrm{em} G$. Portanto, os triângulos que têm um vértice em comum com $T$ se intersectam dois a dois, isto é, $T$ é localmente ótimo em $G$, o que contradiz (3.25).

Teorema 3.8 Em cada iteração do algoritmo $\mathrm{VT}_{k}$, temos que $\left|B_{H}\right| \leq 2$.

Demonstração. Suponha que $B_{H} \neq \emptyset$ e considere $T$ um triângulo em $B_{H}$. Pela definição de $B_{H}$, o triângulo $T$ forma uma borboleta com um triângulo $\widetilde{T} \notin \mathcal{T}_{H}$.

Se $d_{H}(T)=4$, então usando os fatos de que $\Delta(G)=4$ e $T \cup \widetilde{T}$ é uma borboleta em $G$, concluímos que o subgrafo $H$ induzido pelo triângulo $T$ e pelos triângulos de $H$ que intersectam $T$ é isomorfo ao grafo da Figura 3.6(a). Note que $y$ e $z$ são saturados. Ademais, como $\Delta(G)=4$, o vértice $t$ (resp. $w$ ) do grafo mostrado na Figura 3.6(a) não está na interseção de dois triângulos que formam uma borboleta em $G$. Logo, se $H$ é isomorfo ao grafo da Figura 3.6(a), então $[y, t, w]$ é localmente ótimo em $G$, o que contradiz (3.25). Portanto, como $v_{T \widetilde{T}}, y$ e $z$ já são saturados, existe um vértice $p$ adjacente a $t$ e $w$. Note que agora $t$ e $w$ são saturados também. Do Lema 3.7 segue que $d_{G}(p) \geq 3$, ou seja, o triângulo $[t, p, w]$ também está em $B_{H}$, e portanto $\left|B_{H}\right|=2$. Observe que $H$ é isomorfo ao grafo da Figura 3.6(b).

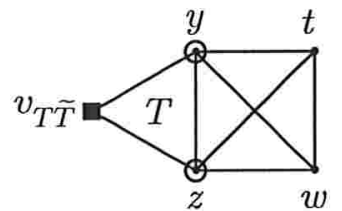

(a)

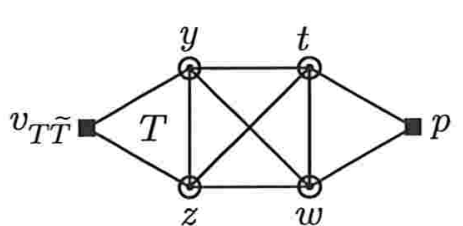

(b)

Figura 3.6: Os possíveis casos quando $d_{H}(T)=4$.

Se, entretanto, $d_{H}(T)=3$, então como $\Delta(G)=4$ e $T \cup \widetilde{T}$ é uma borboleta em $G$, o subgrafo de $H$ induzido pelo triângulo $T$ e pelos triângulos de $H$ que intersectam $T$ é isomorfo ao grafo da Figura 3.7(a) (note que $y, p$ e $z$ são saturados). Se $H$ é isomorfo ao grafo da Figura 3.7(a), então $[y, p, z]$ é localmente ótimo em $G$, uma contradição. Portanto, 
$t w \in E_{H}$. Se $d_{G}(t)=d_{G}(w)=3$, então $[t, p, w]$ é localmente ótimo em $G$, novamente uma contradição. Logo, existe um vértice $l$ adjacente a $t$ e a $w$. Note que agora $t$ e $w$ são saturados também. Do Lema 3.7 segue que $d_{G}(l) \geq 3$, isto é, o triângulo $[t, l, w]$ também pertence a $B_{H}$, e portanto $\left|B_{H}\right|=2$. Observe que $H$ é isomorfo ao grafo da Figura 3.7(b).

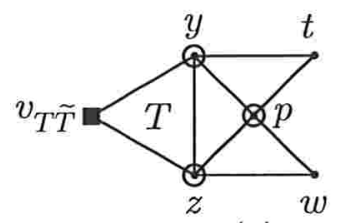

(a)

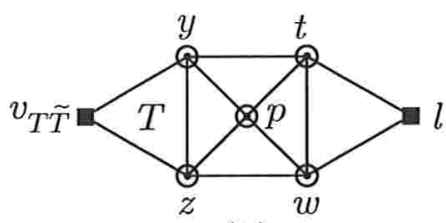

(b)

Figura 3.7: Os possíveis casos quando $d_{H}(T)=3$.

Se $d_{H}(T)=2$ e o subgrafo de $H$ induzido pelo triângulo $T$ e pelos triângulos de $H$ que intersectam $T$ é isomorfo ao grafo da Figura 3.8(a), então claramente $t w \notin E_{H}$. Além disso, $T \cup \widetilde{T}$ é uma borboleta em $G$ e $v_{T \widetilde{T}}, z, y$ são saturados. Logo, do Lema 3.7 temos que $[y, t, z]$ (resp. $[y, w, z]$ ) forma uma borboleta com um triângulo que não está em $H$, com o vértice $t$ (resp. $w$ ) na interseção dos triângulos que formam tal borboleta. Portanto, da definição de $H$, segue que neste caso $H$ é isomorfo ao grafo da Figura 3.8(a). Mas então $H$ não tem nenhum triângulo de grau maior que $3 \mathrm{em} G$, uma contradição. Logo, o subgrafo de $H$ induzido por $T$ e pelos triângulos de $H$ que intersectam $T$ é isomorfo ao grafo da Figura 3.8(b).

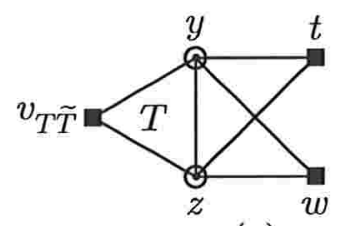

(a)

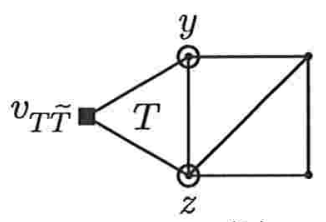

(b)

Figura 3.8: $\operatorname{Se} d_{H}(T)=2$, o subgrafo de $H$ induzido por $T$ e pelos triângulos de $H$ que intersectam $T$ não é isomorfo ao grafo em (a), mas ao grafo em (b). Em (b), o vértice $y$ é saturado, pois $d_{H}(T)=2$.

Agora, como $T \cup \widetilde{T}$ é uma borboleta em $G, \Delta(G)=4, G$ é irredundante e $H$ não contém borboletas, temos as seguintes duas possibilidades.

- $H$ é isomorfo ao grafo da Figura 3.9(a). Note que $H$ pode ter um número par ou ímpar de triângulos (a configuração do grafo $H$ com número par de triângulos é similar à do grafo da Figura 3.9(a)). Como $H$ tem um triângulo com grau em $G$ maior que 3, o grafo $H$ tem pelo menos 7 vértices. Ademais, pelo Lema $3.7, d_{G}(x) \geq 3$, ou seja, $\left|B_{H}\right|=2$ para esse grafo.

- $H$ é isomorfo ao grafo da Figura 3.9(b) (linhas cheias indicam arestas em $H$ ). Note que $H$ pode ter número par ou ímpar de triângulos (a configuração do grafo $H$ com número par de triângulos é similar à do grafo da Figura 3.9(b)). Observe ainda que $x y \notin E_{G} \mathrm{e}$ 


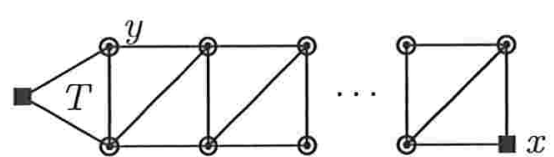

(a)

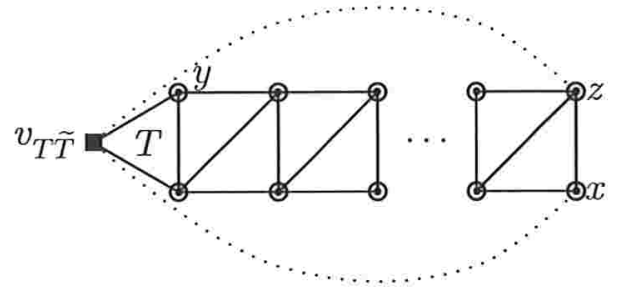

(b)

Figura 3.9: Configurações possíveis quando $d_{H}(T)=2$. Em (a), $\left|B_{H}\right|=2$ e o grafo $H$ tem pelo menos 7 vértices. Em (b), as linhas cheias indicam arestas em $H$. O grafo (b) tem pelo menos 9 vértices, $G\left[V_{H}\right]$ é uma componente de $G, x y$ não é aresta de $G$ e $\left|B_{H}\right|=1$.

que $T$ forma uma borboleta com o triângulo $\left[v_{T \widetilde{T}}, z, x\right]$. Mostramos em seguida que

$$
G\left[V_{H}\right] \text { é uma componente de } G \text {. }
$$

Suponha que (3.26) não ocorra. Como todos os vértices do grafo da Figura 3.9(b) têm grau 4 em $G$, exceto $x$ e $y$, então existe um vértice $w \notin V_{H}$ tal que, sem perda de generalidade, $x w \in E_{G}$. Da limitação do grau dos vértices do grafo e o fato de que $x y \notin E_{G}$, concluímos que $x w$ não é aresta de nenhum triângulo, uma contradição. Logo, a afirmação (3.26) é válida. Temos então que $\left|V_{H}\right| \geq 9$ (caso contrário, $H$ teria uma coleção $\mathcal{T},|\mathcal{T}| \leq 2$, localmente ótima em $G$ ). Note ainda que $\left|B_{H}\right|=1$.

Provamos portanto que $\left|B_{H}\right| \leq 2$ em todos os casos possíveis.

Lema 3.9 Em cada iteração do algoritmo $\mathrm{VT}_{k}$, se $B_{H}=\emptyset$ então $d_{H}(x) \geq 3$ para cada $x \in V_{H}$ e $H$ é uma componente de $G$.

Demonstração. Suponha que exista $x \in V_{H} \operatorname{com} d_{H}(x)=2$. Então o Lema 3.7 implica que existe uma aresta $x w$ que não está em $E_{H}$. Do fato que $G$ é irredundante, temos que $x w$ é aresta de um triângulo $\widetilde{T} \in \mathcal{T}_{G} \backslash \mathcal{T}_{H}$. Como $B_{H}=\emptyset$, então $\widetilde{T}$ não induz nenhuma borboleta com um triângulo de $H$. Além disso, $x$ está em $V_{H}$ e portanto, pela definição de $H$, temos que $\tilde{T} \in \mathcal{T}_{H}$, uma contradição. Similarmente, temos que $H$ é uma componente de $G$.

Teorema 3.10 Para cada iteração do algoritmo $\mathrm{VT}_{k}$, se $\left|B_{H}\right| \leq 1$, então $G\left[V_{H}\right]$ é uma componente de $G$ e $\mathrm{Sol}_{H}$ é uma solução ótima nessa componente (onde $\mathrm{Sol}_{H}$ é o conjunto como definido nas linhas 12 e 13 do algoritmo $\mathrm{VT}_{k}$ ).

Demonstração. Como vimos na prova do Teorema 3.8 , se $\left|B_{H}\right|=1$, então $H$ é isomorfo ao grafo da Figura 3.9(b) e $G\left[V_{H}\right]$ é uma componente de $G$. Note que neste caso $\operatorname{Adota}(H)$ é um empacotamento de $\mathcal{T}_{V}(H)$ que cobre todos os vértices de grafo $H$, exceto no máximo dois, e portanto $\operatorname{Adota}(H)$ é uma solução ótima no componente $G\left[V_{H}\right]$.

Suponha agora que $B_{H}=\emptyset$. Do Lema 3.9 , segue que $H$ é uma componente de $G$, e portanto $\left|V_{H}\right| \geq 9$ (caso contrário, $G$ teria uma coleção $\mathcal{T},|\mathcal{T}| \leq 2$, localmente ótima). 
Se cada par de triângulos em $H$ tem dois vértices em comum, então $H$ é isomorfo ao grafo da Figura 3.10. Portanto, $H$ não tem nenhum triângulo $T \operatorname{com} d_{G}(T)>3$, uma contradição.

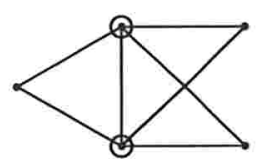

Figura 3.10: O caso em que todo par de triângulos em $H$ tem dois vértices em comum não é possível.

Sejam então $T_{1}=[x, y, z]$ e $T_{2}=[x, t, r]$ triângulos em $H$ com somente um vértice em comum. Como $H$ não tem borboletas, usando o fato (3.24), podemos supor, sem perda de generalidade, que $y t \in E_{H}$. Agora, temos as seguintes possibilidades.

(i) $r z \in E_{H}$. Como $\left|V_{H}\right|>5$, usando a limitação do grau dos vértices do grafo e o fato de que o grafo é irredundante, temos que existe um vértice $p$ adjacente a, sem perda de generalidade, $y$ e a $z$. Do Lema 3.9 , temos que $d_{H}(p) \geq 3$. Portanto, como $H$ é irredundante e não tem borboletas, e $x, y, z$ já são saturados, segue que $p t \in E_{H}$ ou $p r \in E_{H}$. Se ambos $p t$ e $p r$ são arestas de $H$, então todos os vértices de $H$ são saturados, e $\left|V_{H}\right| \leq 8$, uma contradição com o fato de que $\left|V_{H}\right| \geq 9$. Se, no entanto, $p t \in E_{H}$, $p r \notin E_{H}$ (veja a Figura 3.11) então não existe um vértice $k$ adjacente a $r$ ou a $p$. De fato, suponha que exista um novo vértice $k$ tal que, sem perda de generalidade, $r k \in E_{G}$. Da limitação do grau dos vértices e do fato de que $p r \notin E_{H}$, segue que $r k$ não é aresta de nenhum triângulo, uma contradição. Portanto, novamente, $\left|V_{H}\right| \leq 8$, uma contradição. Similarmente se $p t \notin E_{H}, p r \in E_{H}$.

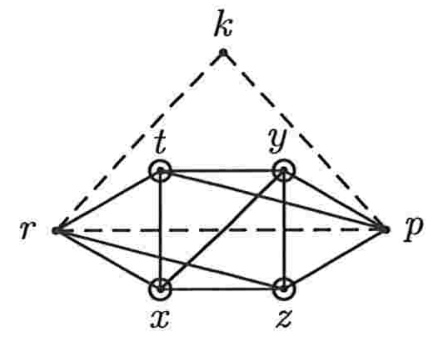

Figura 3.11: $\mathrm{O}$ grafo $H$ quando $r z$, $p t$ estão em $E_{H}$, e $p r$ não está em $E_{H}$. As linhas tracejadas não podem ser arestas de $H$.

(ii) $r z \notin E_{H}, r y \in E_{H}$. Se $t z \in E_{H}$, então claramente $x, y, t$ são saturados. Como $r z \notin E_{H}$, similarmente como acima, concluímos que não existe vértice $k$ adjacente a $r$ ou a $z$. Portanto, $H$ é isomorfo ao grafo da Figura 3.12(a) e $\left|V_{H}\right| \leq 8$, uma contradição. Se, entretanto, $t z \notin E_{H}$, então de (3.25) temos que $\left|V_{H}\right|>5$. Então, como $t z, r z \notin E_{H}$, o único jeito de expandir o grafo é por um novo vértice $w$ adjacente a $t$ e a $r$. Agora, do Lema 3.9 , temos que $d_{H}(z) \geq 3$ e $d_{H}(w) \geq 3$. Como $x, y, r, t$ já são saturados, a única possível configuração de $H$ é mostrada na Figura 3.12(b). Mas então $H$ tem uma borboleta $[r, t, w] \cup[w, k, z]$, uma contradição. 


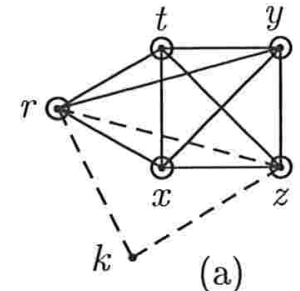

(a)

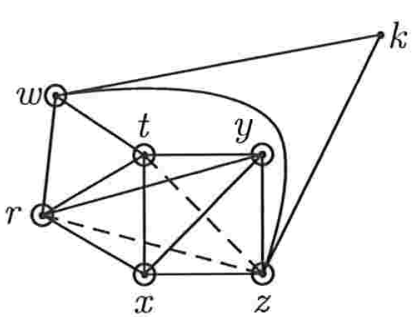

(b)

Figura 3.12: Os casos em que $r z$ não está em $E_{H}$, e ry está em $E_{H}$.

(iii) $r z, r y \notin E_{H}, t z \in E_{H}$. Note que este caso é equivalente ao caso (ii) quando $t z \notin E_{H}$.

(iv) $r z, r y, t z \notin E_{H}$.

(1) Existe um vértice $p$ adjacente a $t$ e a $y$ em $H$. Como $x, y, t$ são saturados, do Lema 3.9 e do fato de que $H$ não tem borboletas, temos que $r p, p z \in E_{H}$, e então novamente $\left|V_{H}\right| \leq 8$, uma contradição (veja a Figura 3.13).

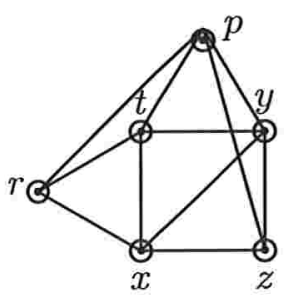

Figura 3.13: $\mathrm{O}$ caso em que $r z, r y, t z$ não são arestas de $H$ e existe um vértice $p$ adjacente a $t$ e a $y$ em $H$.

(2) Não existe vértice adjacente a $t$ e a $y$ em $H$. Como $\left|V_{H}\right|>5$, existe um vértice $p$ adjacente a, sem perda de generalidade, $y$ e a $z$. Como o caso (i) não é possível, temos que $t p \notin E_{H}$. De fato, se $t p \in E_{H}$, teríamos um grafo (o grafo da Figura 3.14(a) induzido pelos vértices $x, y, z, t, p)$ isomorfo ao grafo da Figura 3.11 induzido pelos vértices $x, y, z, t, r$ (o que é, como provamos, impossível).

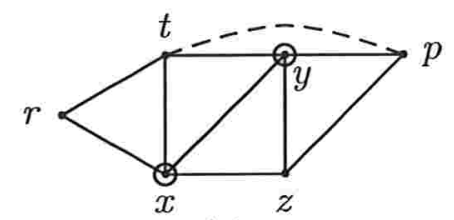

(a)

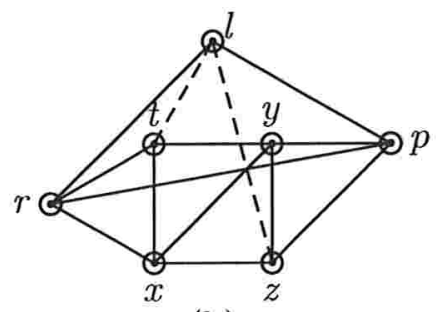

(b)

Figura 3.14: Casos em que $r z, r y, t z$ não são arestas de $H$ e não existe vértice adjacente a $t$ e a $y$ em $H$.

Suponha agora que não exista nenhum vértice adjacente a ambos $p$ e $z$, ou adjacente a ambos $r$ e $t$. Então, do Lema 3.9 e do fato de que $t p, r z \notin E_{H}$, segue que $p r \in E_{H}$. 
Como $H$ é irredundante e $t p, r z \notin E_{H}$, concluímos que existe um triângulo $[p, r, l]$ em $H$ que contém a aresta $p r$. Agora, nossa hipótese (de que não existe vértice adjacente a ambos $p$ e $z$, ou adjacente a ambos $r$ e $t$ ) implica que $l t, l z \notin E_{H}$, e portanto o grafo $H$ tem uma borboleta $[r, p, l] \cup[r, t, x]$ (veja a Figura 3.14(b)), uma contradição. Concluímos portanto que existe um vértice $l$ adjacente a, sem perda de generalidade, $p$ e a $z$ (Figura 3.15(a)).

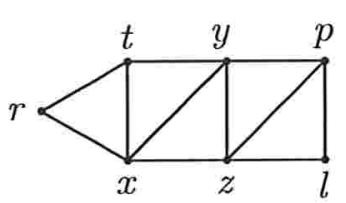

(a)

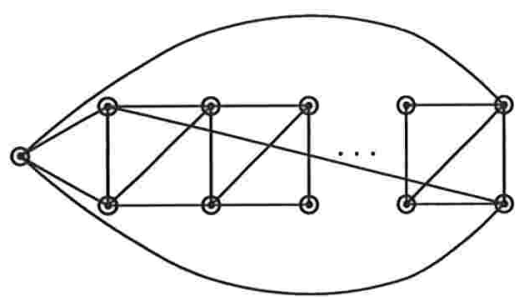

(b)

Figura 3.15: Um grafo $H$ que tem o grafo em (a) como subgrafo e satisfaz $B_{H}=\emptyset$ é isomorfo ao grafo em (b).

Note que o grafo $H$ que tem o grafo na Figura 3.15(a) como subgrafo e satisfaz $B_{H}=\emptyset$ é isomorfo ao grafo da Figura 3.15(b). Observe que $H$ pode ter um número par ou ímpar de triângulos (a configuração do grafo $H$ com número par de triângulos é similar à grafo da Figura 3.15(b)). Como todos os vértices nesse grafo são saturados, $H$ é uma componente de $G$, e portanto $\left|V_{H}\right| \geq 9$ (caso contrário, $H$ teria uma coleção $\mathcal{T},|\mathcal{T}| \leq 2$, localmente ótima em $G$ ). Observe que, se $\widetilde{T}$ é um triângulo em $H$, então $H-V_{\widetilde{T}}$ é isomorfo ao grafo com linhas cheias mostrado na Figura 3.9(b) (mas agora com pelo menos 6 vértices). Portanto, $\operatorname{Adota}\left(H-V_{\widetilde{T}}\right)$ é um empacotamento de triângulos disjuntos nos vértices em $H-V_{\widetilde{T}}$ que cobre todos os vértices de $H-V_{\widetilde{T}}$, exceto no máximo dois. Como $\left|V_{H}\right|=\left|V_{H-V_{\widetilde{T}}}\right|+3$, temos que $\widetilde{T} \cup \operatorname{Adota}\left(H-V_{\widetilde{T}}\right)$ é uma solução ótima em $H$.

Corolário 3.11 Em cada iteração do algoritmo $\mathrm{VT}_{k}$, o grafo $H$ é isomorfo a um dos grafos na Figura 3.16.

Demonstração. Segue das provas dos Teoremas 3.8 e 3.10 .

Teorema 3.12 Se $\mathcal{T}$ é uma coleção localmente ótima em $G$, então Aceita $(\mathcal{T})$ preserva a razão de aproximação do algoritmo $\mathrm{VT}_{k}$.

Demonstração. Notamos, inicialmente, que existe um empacotamento máximo de $\mathcal{T}_{V}(G)$ que contém $\mathcal{T}$. De fato, se um empacotamento máximo de $\mathcal{T}_{V}(G)$ não contém $\mathcal{T}$, podemos substituir os triângulos que intersectam algum triângulo em $\mathcal{T}$, pelos triângulos de $\mathcal{T}$. Logo, para $G^{\prime}:=G-\bigcup_{T \in \mathcal{T}} V_{T}$ temos opt $\left(G^{\prime}\right) \geq \operatorname{opt}(G)-|\mathcal{T}|$. Portanto, se um empacotamento $\mathcal{A}$ de $\mathcal{T}_{V}\left(G^{\prime}\right)$ satisfaz $\operatorname{opt}\left(G^{\prime}\right) \leq \rho|\mathcal{A}|(\operatorname{com} \rho \geq 1)$, então $\operatorname{opt}(G) \leq \operatorname{opt}\left(G^{\prime}\right)+|\mathcal{T}| \leq \rho|\mathcal{A}|+|\mathcal{T}| \leq$ $\rho|\mathcal{A} \cup \mathcal{T}|$. 


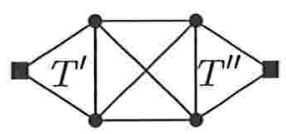

(a)

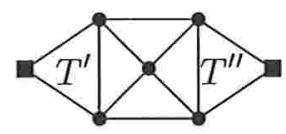

(b)

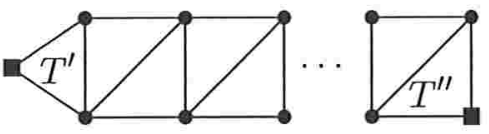

(c)

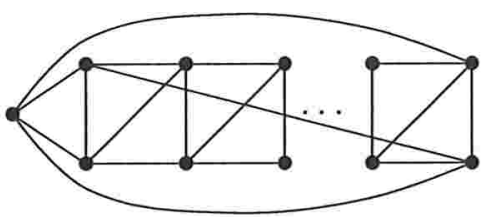

(e)

(d)

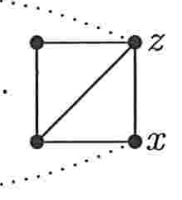

Figura 3.16: Possíveis configurações do grafo $H$. Cada vértice quadrado é comum a dois triângulos de $G$ cuja união é uma borboleta. O grafo (c) tem pelo menos 7 vértices. Os grafos (d) e (e) têm pelo menos 9 vértices e $G\left[V_{H}\right]$ é uma componente de $G$ (em (d) $x y$ não é aresta de $G$. As linhas pontilhadas indicam arestas que estão em $E_{G}$, mas não em $E_{H}$ ). Note que, em (d), $T^{\prime}$ induz uma borboleta com o triângulo $\left[v_{T^{\prime} \tilde{T}^{\prime}}, x, z\right]$. Em (c), (d) e (e), $H$ pode ter um número par ou ímpar de triângulos (a configuração do grafo $H$ com número par de triângulos é similar ao grafo mostrado nesta figura).

Corolário 3.13 Se $\mathcal{T}$ é uma coleção localmente ótima em um grafo $G$ e $\mathcal{A}$ é um empacotamento máximo de triângulos disjuntos nos vértices em $G-\bigcup_{T \in \mathcal{T}} V_{T}$, então $\mathcal{A} \cup \mathcal{T}$ é um empacotamento máximo de $\mathcal{T}_{V}(G)$.

Demonstração. Segue diretamente da prova do Teorema 3.12 (tome $\rho=1$ ).

Teorema 3.14 Redução $(H)$ preserva a razão de aproximação do algoritmo $\mathrm{VT} 4_{k}$.

Demonstração. Considere qualquer iteração do algoritmo para a qual existe um triângulo em $G$ com grau maior que 3 (onde $G$ é o grafo na linha 5 ) e $\left|B_{H}\right|=2$. Então $H$ é um dos grafos da Figura 3.16(a), (b) ou (c). Note que

se $K$ é qualquer um dos grafos $H-V_{T^{\prime}}-V_{T^{\prime \prime}}, H-V_{T^{\prime}}-v_{T^{\prime \prime} \widetilde{T}^{\prime \prime}}, H-V_{T^{\prime \prime}}-v_{T^{\prime} \widetilde{T}^{\prime}}$,

$H-v_{T^{\prime} \widetilde{T}^{\prime}}-v_{T^{\prime \prime} \widetilde{T}^{\prime \prime}}$, então $\operatorname{Adota}(K)$ é um empacotamento máximo de $\mathcal{T}_{V}(K)$.

Conseqüentemente, $\operatorname{Sol}_{T^{\prime} T^{\prime \prime}}$ é um empacotamento máximo de $\mathcal{T}_{V}(H)$ que contém $T^{\prime}$ e $T^{\prime \prime}$, $\mathrm{Sol}_{T^{\prime} \bar{T}^{\prime \prime}}$ é um empacotamento máximo de $\mathcal{T}_{V}(H)$ que contém $T^{\prime}$ mas não $T^{\prime \prime}$, etc. Ademais, para $H$ isomorfo a algum dos grafos da Figura 3.16(a), (b) ou (c), as igualdades listadas na definição da $\operatorname{Redução~}(H)$ cobrem todas as possíveis relações entre as cardinalidades dos conjuntos $\operatorname{Sol}_{T^{\prime} T^{\prime \prime}}, \operatorname{Sol}_{T^{\prime} \bar{T}^{\prime \prime}}, \operatorname{Sol}_{\bar{T}^{\prime} T^{\prime \prime}} \mathrm{e} \mathrm{Sol}_{\bar{T}^{\prime} \bar{T}^{\prime \prime}}$.

Suponha que a igualdade em 3(a) seja satisfeita. Da definição do grafo $H$ e do fato de que $T^{\prime} \cup \widetilde{T}^{\prime}$ (resp. $T^{\prime \prime} \cup \widetilde{T}^{\prime \prime}$ ) é uma borboleta em $G$, concluímos que os triângulos em $H$ que têm um vértice em comum com $T^{\prime}$ (resp. $T^{\prime \prime}$ ) se intersectam dois a dois. Além disso, vimos que $T^{\prime}$ e $T^{\prime \prime}$ são disjuntos, e conseqüentemente, $\left\{T^{\prime}, T^{\prime \prime}\right\}$ é localmente ótimo em $H$. Logo, da afirmação (3.27) (tome $K=H-V_{T^{\prime}}-V_{T^{\prime \prime}}$ ) e do Corolário 3.13, segue que $\operatorname{Sol}_{T^{\prime} T^{\prime \prime}}$ é um empacotamento máximo de $\mathcal{T}_{V}(H)$. Como $\left|\mathrm{Sol}_{T^{\prime} T^{\prime \prime}}\right|=\left|\mathrm{Sol}_{\bar{T}^{\prime}} \bar{T}^{\prime \prime}\right|$, então $\operatorname{Sol}_{\bar{T}^{\prime} \bar{T}^{\prime \prime}}$ é também um empacotamento máximo de $\mathcal{T}_{V}(H)$. Note que $\left\{T \in \mathcal{T}_{G}: T\right.$ intersecta um triângulo em $\left.\operatorname{Sol}_{\bar{T}^{\prime} \bar{T}^{\prime \prime}}\right\} \subseteq$ 
$\mathcal{T}_{H}$. Além disso, $\mathcal{T}_{H} \subseteq\left\{T \in \mathcal{T}_{G}: T\right.$ intersecta um triângulo em $\left.\operatorname{Sol}_{\bar{T}^{\prime}} \bar{T}^{\prime \prime}\right\}$, pois, caso contrário, $\mathrm{Sol}_{\bar{T}^{\prime} \bar{T}^{\prime \prime}}$ não seria um empacotamento máximo de $\mathcal{T}_{V}(H)$. Segue que $\mathrm{Sol}_{\bar{T}^{\prime} \bar{T}^{\prime \prime}}$ é localmente ótimo em $G$, e pelo Teorema $3.12, \operatorname{Aceita}\left(\mathrm{Sol}_{\bar{T}^{\prime} \bar{T}^{\prime \prime}}\right)$ preserva a razão de aproximação do algoritmo.

Suponha agora que a igualdade em $3\left(\right.$ a) não é satisfeita e que $\widetilde{T}^{\prime}=\widetilde{T}^{\prime \prime}$. Seja $\mathcal{A}$ um empacotamento máximo de $\mathcal{T}_{V}\left(H \cup \widetilde{T}^{\prime}\right)$. Se $\widetilde{T}^{\prime} \in \mathcal{A}$, então claramente, $|\mathcal{A}|=\left|\operatorname{Sol}_{\bar{T}^{\prime}} \bar{T}^{\prime \prime}\right|+1$; se $\widetilde{T}^{\prime} \notin \mathcal{A}$, então $|\mathcal{A}|=\left|\operatorname{Sol}_{T^{\prime} T^{\prime \prime}}\right|\left(\operatorname{pois}\left|\operatorname{Sol}_{T^{\prime} \bar{T}^{\prime \prime}}\right|,\left|\operatorname{Sol}_{\bar{T}^{\prime} T^{\prime \prime}}\right|,\left|\operatorname{Sol}_{\bar{T}^{\prime} \bar{T}^{\prime \prime}}\right| \leq\left|\operatorname{Sol}_{T^{\prime} T^{\prime \prime}}\right|\right)$. A igualdade em 3(a) não é satisfeita, ou seja, uma das igualdades em 3(c) ou 3(d) é satisfeita. Logo, $\left|\mathrm{Sol}_{T^{\prime} T^{\prime \prime}}\right|=$ $\left|\operatorname{Sol}_{\bar{T}^{\prime} \bar{T}^{\prime \prime}}\right|+1$. Portanto, $\operatorname{Sol}_{T^{\prime} T^{\prime \prime}}$ é um empacotamento máximo de $\mathcal{T}_{V}\left(H \cup \widetilde{T}^{\prime}\right)$. Agora, analogamente como acima, concluímos que $\left\{T \in \mathcal{T}_{G}: T\right.$ intersecta um triângulo em $\left.\operatorname{Sol}_{T^{\prime} T^{\prime \prime}}\right\}=$ $\mathcal{T}_{H} \cup \widetilde{T}^{\prime}$. Logo, Sol $_{T^{\prime} T^{\prime \prime}}$ é localmente ótimo em $G$, e o Teorema 3.12 implica que Aceita $\left(\operatorname{Sol}_{T^{\prime} T^{\prime \prime}}\right)$ preserva a razão de aproximação do algoritmo.

Se a igualdade em $3\left(\right.$ c) ou $3\left(\right.$ d) é satisfeita e $\widetilde{T}^{\prime} \neq \widetilde{T}^{\prime \prime}$, definimos $G_{A}$ como o grafo $G$ antes da execução de Redução $1(H)$ (resp. Redução $2(H)$ ), e $G_{P}$ como o grafo $G$ logo após a execução da Redução $1(H)$ (resp. Redução $2(H)$ ). Seja ainda $\mathcal{A}_{P}$ um empacotamento maximal de triângulos disjuntos nos vértices em $G_{P}$.

Suponha que a igualdade em $3(\mathrm{c})$ seja satisfeita e que $\widetilde{T}^{\prime} \neq \widetilde{T}^{\prime \prime}$. Mostramos inicialmente que

$$
\operatorname{opt}\left(G_{P}\right) \geq \operatorname{opt}\left(G_{A}\right)-\left|\operatorname{Sol}_{\bar{T}^{\prime} \bar{T}^{\prime \prime}}\right|+1 \text {. }
$$

Seja $\mathcal{T}_{A}^{*}$ um conjunto de triângulos que é um empacotamento máximo de $\mathcal{T}_{V}\left(G_{A}\right)$. Se $T^{\prime}, T^{\prime \prime} \in$ $\mathcal{T}_{A}^{*}$, então $\left|\mathcal{T}_{A}^{*} \cap \mathcal{T}_{H}\right|=\left|\operatorname{Sol}_{T^{\prime} T^{\prime \prime}}\right|$, pois $\operatorname{Sol}_{T^{\prime} T^{\prime \prime}}$ é um empacotamento máximo de $\mathcal{T}_{V}(H)$ que contém $T^{\prime}$ e $T^{\prime \prime}$; logo, $\mathcal{T}_{A}^{*} \backslash\left(\mathcal{T}_{H} \backslash\left\{T^{\prime}, T^{\prime \prime}\right\}\right)$ é um empacotamento de $\mathcal{T}_{V}\left(G_{P}\right)$ cujo tamanho é $\operatorname{opt}\left(G_{A}\right)-\left|\operatorname{Sol}_{T^{\prime} T^{\prime \prime}}\right|+2=\operatorname{opt}\left(G_{A}\right)-\left|\operatorname{Sol}_{\bar{T}^{\prime} \bar{T}^{\prime \prime}}\right|+1 . \operatorname{Se} T^{\prime}, T^{\prime \prime} \notin \mathcal{T}_{A}^{*}$, então $\left|\mathcal{T}_{A}^{*} \cap \mathcal{T}_{H}\right|=\left|\operatorname{Sol}_{\bar{T}^{\prime} \bar{T}^{\prime \prime}}\right|$, e portanto $\left(\mathcal{T}_{A}^{*} \backslash \mathcal{T}_{H}\right) \cup T_{H}$ é um empacotamento de $\mathcal{T}_{V}\left(G_{P}\right)$ de cardinalidade $\operatorname{opt}\left(G_{A}\right)$ $\left|\mathrm{Sol}_{\bar{T}^{\prime} \bar{T}^{\prime \prime}}\right|+1$. Se, entretanto, $T^{\prime} \in \mathcal{T}_{A}^{*}, T^{\prime \prime} \notin \mathcal{T}_{A}^{*}$, então $\mathcal{T}_{A}^{*} \backslash\left(\mathcal{T}_{H} \backslash T^{\prime}\right)$ é um empacotamento de $\mathcal{T}_{V}\left(G_{P}\right)$ de tamanho $\operatorname{opt}\left(G_{A}\right)-\left|\operatorname{Sol}_{T^{\prime} \bar{T}^{\prime \prime}}\right|+1=\operatorname{opt}\left(G_{A}\right)-\left|\operatorname{Sol}_{\bar{T}^{\prime} \bar{T}^{\prime \prime}}\right|+1$. Similarmente se $T^{\prime} \notin \mathcal{T}_{A}^{*}, T^{\prime \prime} \in \mathcal{T}_{A}^{*}$. Portanto, a afirmação (3.28) é válida.

Note que, como $\mathcal{A}_{P}$ é maximal, pelo menos um dos triângulos $T^{\prime}, T^{\prime \prime}, T_{H}$ está em $\mathcal{A}_{P}$. Definimos agora o conjunto $\mathcal{A}_{A}$ (de acordo com Restauração $(H)$ ).

$$
\mathcal{A}_{A}:= \begin{cases}\mathcal{A}_{P} \backslash T_{H} \cup \operatorname{Sol}_{\bar{T}^{\prime}} \bar{T}^{\prime \prime} & \text { se } T_{H} \in \mathcal{A}_{P} \\ \mathcal{A}_{P} \cup \operatorname{Sol}_{T^{\prime} T^{\prime \prime}} & \text { se } T^{\prime}, T^{\prime \prime} \in \mathcal{A}_{P} \\ \mathcal{A}_{P} \cup \operatorname{Sol}_{T^{\prime} \bar{T}^{\prime \prime}} & \text { se } T^{\prime} \in \mathcal{A}_{P}, T^{\prime \prime} \notin \mathcal{A}_{P} \\ \mathcal{A}_{P} \cup \operatorname{Sol}_{\bar{T}^{\prime} T^{\prime \prime}} & \text { se } T^{\prime} \notin \mathcal{A}_{P}, T^{\prime \prime} \in \mathcal{A}_{P}\end{cases}
$$

Mostramos em seguida que

$$
\left|\mathcal{A}_{A}\right|=\left|\mathcal{A}_{P}\right|+\left|\mathrm{Sol}_{\bar{T}^{\prime} \bar{T}^{\prime \prime}}\right|-1 \mathrm{e}
$$

$$
\mathcal{A}_{A} \text { é um empacotamento maximal em } \mathcal{T}_{V}\left(G_{A}\right) \text {. }
$$

Se $T_{H} \in \mathcal{A}_{P}$, então é claro que $\left|\mathcal{A}_{A}\right|=\left|\mathcal{A}_{P}\right|+\left|\operatorname{Sol}_{\bar{T}^{\prime} \bar{T}^{\prime \prime}}\right|-1$. Se $T^{\prime}, T^{\prime \prime} \in \mathcal{A}_{P}$, então $\left|\mathcal{A}_{A}\right|=$ $\left|\mathcal{A}_{P}\right|+\left|\mathrm{Sol}_{T^{\prime} T^{\prime \prime}}\right|-2=\left|\mathcal{A}_{P}\right|+\left|\mathrm{Sol}_{\bar{T}^{\prime} \bar{T}^{\prime \prime}}\right|-1$. Se, no entanto, $T^{\prime} \in \mathcal{A}_{P}, T^{\prime \prime} \notin \mathcal{A}_{P}$, então $\left|\mathcal{A}_{A}\right|=\left|\mathcal{A}_{P}\right|+\left|\operatorname{Sol}_{T^{\prime} \bar{T}^{\prime \prime}}\right|-1=\left|\mathcal{A}_{P}\right|+\left|\operatorname{Sol}_{\bar{T}^{\prime} \bar{T}^{\prime \prime}}\right|-1$. Similarmente se $T^{\prime} \notin \mathcal{A}_{P}, T^{\prime \prime} \in \mathcal{A}_{P}$. Logo, a afirmação (3.29) é válida. Da afirmação (3.27) e da maximalidade de $\mathcal{A}_{P}$, segue que $\mathcal{A}_{A}$ é um empacotamento maximal de $\mathcal{T}_{V}\left(G_{A}\right)$ em todos os casos. Portanto, podemos concluir que (3.30) é válido. 
Se $\operatorname{opt}\left(G_{P}\right) \leq \rho\left|\mathcal{A}_{P}\right|$ (para algum $\rho \geq 1$ ), então de (3.28) e (3.29) temos $\operatorname{opt}\left(G_{A}\right) \leq$ $\operatorname{opt}\left(G_{P}\right)+\left|\operatorname{Sol}_{\bar{T}^{\prime} \bar{T}^{\prime \prime}}\right|-1 \leq \rho\left|\mathcal{A}_{P}\right|+\left|\operatorname{Sol}_{\bar{T}^{\prime} \bar{T}^{\prime \prime}}\right|-1=\rho\left(\left|\mathcal{A}_{A}\right|-\left|\operatorname{Sol}_{\bar{T}^{\prime} \bar{T}^{\prime \prime}}\right|+1\right)+\left|\operatorname{Sol}_{\bar{T}^{\prime} \bar{T}^{\prime \prime}}\right|-1$. Note que $\left|\operatorname{Sol}_{\bar{T}^{\prime} \bar{T}^{\prime \prime}}\right| \geq 1$ e portanto opt $\left(G_{A}\right) \leq \rho\left|\mathcal{A}_{A}\right|$.

Observamos que o algoritmo MIS $3_{k}$ devolve um conjunto estável maximal e, portanto, imediatamente após a linha $15, \mathcal{A}^{*}$ é um empacotamento maximal de $\mathcal{T}_{V}(G)$. Notamos ainda que as restaurações dos subgrafos são feitas na ordem reversa das respectivas reduções. Portanto, usando (3.30), por indução podemos deduzir que, para cada Redução $1(H)$ aplicada no algoritmo, se $G_{P}$ é o grafo como definido previamente, então $\mathcal{A}_{P}$ é um empacotamento maximal de $\mathcal{T}_{V}\left(G_{P}\right)$. Segue, portanto, que Restauração $(H)$ está bem definida, ou seja, pelo menos um dos triângulos $T^{\prime}, T^{\prime \prime}, T_{H}$ pertence a $\mathcal{A}^{*}$ antes da execução de $\operatorname{Restauração~}(H)$. Ademais, como vimos, $\mathcal{A}_{A}$ é um empacotamento de $\mathcal{T}_{V}\left(G_{A}\right)$, e $\operatorname{opt}\left(G_{P}\right) \leq \rho\left|\mathcal{A}_{P}\right|$ implica em $\operatorname{opt}\left(G_{A}\right) \leq \rho\left|\mathcal{A}_{A}\right|$. Concluímos portanto que cada Redução $1(H)$ (e Restauração $(H)$ correspondente) preserva a razão de aproximação do algoritmo.

A prova do fato de que Redução $2(H)$ (e Restauração $(H)$ correspondente) preserva a razão de aproximação do algoritmo é análoga.

Teorema $3.15 O$ algoritmo $\mathrm{VT}_{k}$ é um algoritmo de $\left(3-\frac{\sqrt{13}}{2}+\frac{13-\sqrt{13}}{52 k}\right)$-aproximação para o problema VTP-4.

Demonstração. De acordo com os Teoremas 3.10, 3.12 e 3.14, todas as reduções aplicadas no algoritmo $\mathrm{VT}_{k} k$ preservam a razão de aproximação. Portanto, a razão de aproximação do algoritmo $\mathrm{VT}_{k}$ é a razão de aproximação do algoritmo $\mathrm{MIS}_{k}$.

\subsubsection{Complexidade computacional do algoritmo VT4 $k$}

Deve-se notar que o algoritmo VT4 $4_{k}$ não precisa fazer uma busca exaustiva dos grafos mostrados na Figura 3.16. Se nenhuma chamada for feita a $\mathrm{MIS}_{k}$, o algoritmo VT4 $4_{k}$ encontra uma solução ótima e pode ser implementado de modo a não consumir tempo maior que $O\left(n_{G}^{3}\right)$, onde $n_{G}$ é a ordem do grafo de entrada. De fato, observe que para encontrar todos os triângulos em $G$ gastamos tempo $O\left(n_{G}\right)$, pois basta, para cada vértice $v$ de $G$, verificar se cada par de arestas que incide em $v$ forma um triângulo. Como $d_{G}(v) \leq 4$, para cada vértice $v$ temos no máximo 6 verificações. Note ainda que como $\Delta(G) \leq 4$, todo triângulo é adjacente a no máximo 9 triângulos. A linha 2 consome tempo $O\left(\left|\mathcal{T}_{G}\right|\right)$, pois, no começo do algoritmo, podemos colocar todos os triângulos com grau maior que 3 em $G$ numa lista, gastando tempo $O\left(\left|\mathcal{T}_{G}\right|\right)$. Cada vez que o procedimento Aceita é executado ou alguma redução é feita no algoritmo, atualizamos esta lista em tempo constante. As linhas 3 e 4 consomem tempo $O\left(\left|\mathcal{T}_{G}\right|^{3}\right)$. De fato, a busca exaustiva de uma coleção $\mathcal{T} \subseteq \mathcal{T}_{G}$ com $|\mathcal{T}| \leq 2$ localmente ótimo em $G$ consome tempo $O\left(\left|\mathcal{T}_{G}\right|^{2}\right)$; ainda, a linha 4 é executada no máximo $O\left(\left|\mathcal{T}_{G}\right|\right)$ vezes. Observe que o grafo $H$ pode ser encontrado em tempo linear em $\left|\mathcal{T}_{G}\right|$. O procedimento $\operatorname{Adota}(H)$ consome tempo $O\left(\left|\mathcal{T}_{G}\right|\right)$. Isto porque encontrar um primeiro triângulo $T$ localmente ótimo em $H$ usando busca exaustiva consome tempo $O\left(\left|\mathcal{T}_{G}\right|\right)$. Devido à estrutura especial do grafo $H$, segue imediatamente que os candidatos para o próximo triângulo localmente ótimo em $H$ são aqueles que são adjacentes a algum triângulo removido anteriormente. Finalmente, como 
$\Delta(G)=4$, do Lema 3.1 temos que $\left|\mathcal{T}_{G}\right| \leq 2 n_{G}$. Logo, o consumo de tempo do algoritmo $\mathrm{VT}_{k}$ é $O\left(n_{G}^{3}\right)$, se nenhuma chamada for feita a $\mathrm{MIS}_{k}$.

O tempo de execução do algoritmo $\mathrm{VT}_{k}$ é dominado pela complexidade da rotina $\mathrm{MIS}_{k}$, que é $O\left(n^{O(k)}\right)$. Portanto, qualquer melhoria na razão de aproximação e/ou tempo de execução do algoritmo para encontrar um subconjunto de vértices independentes de cardinalidade máxima em grafos de grau máximo 3 conduzirá a uma melhoria na qualidade do algoritmo $\mathrm{VT} 4{ }_{k}$.

\subsubsection{Observações finais}

Mostramos em seguida que o algoritmo VT4 $4_{k}$ é superior ao algoritmo simples de busca local, apresentando instâncias do problema VTP para as quais a razão do valor de uma solução ótima sobre o valor devolvido pelo algoritmo de busca local de Hurkens e Schrijver é $\frac{3}{2}+\varepsilon$.

Construiremos um grafo $G$ cujos vértices serão os triângulos de um outro grafo $G^{\prime}$ ( $G^{\prime}$ é a instância desejada para a qual o algoritmo de busca local atinge a razão $\frac{3}{2}+\varepsilon$ ). A construção do grafo $G$ que apresentaremos a seguir foi obtida por Yu e Goldschmidt [30], para mostrar que a razão de aproximação do algoritmo de busca local para o problema do conjunto independente máximo de vértices em grafos livre de $k$-garras é justa.

Seja $r$ um inteiro fixo positivo. Começamos com conjuntos de vértices $S_{1}$ e $T_{1}$ com $\left|S_{1}\right|=$ $\left|T_{1}\right|=1$. Adicionamos conjuntos de vértices $S_{i}$, para $i=2, \ldots, r$, com $\left|S_{i}\right|=2^{i-1}$, conjuntos de vértices $T_{2}^{i}$, para $i=1, \ldots, r-1$, com $\left|T_{2}^{i}\right|=\left|S_{i+1}\right|$, e finalmente um conjunto de vértices $T_{2}^{r}$ com $\left|T_{2}^{r}\right|=\left|S_{r}\right|$. Todos os conjuntos de vértices são disjuntos. As arestas do grafo $G$ são como segue. Existe uma aresta entre o único vértice de $T_{1}$ e o único vértice de $S_{1}$, e uma correspondência unívoca entre os vértices de $T_{2}^{i}$ e os vértices de $S_{i+1}$, para $i=1, \ldots, r-1$ (isto é, existe uma aresta entre cada par correspondente de vértices). Para $i=1, \ldots, r$, colocamos $2\left|S_{i}\right|$ arestas entre o conjunto de vértices $S_{i}$ e o conjunto de vértices $T_{2}^{i}$, de modo que cada vértice no conjunto $S_{i}$ tenha grau exatamente 3 e cada vértice em $T_{2}^{i}$ tenha grau exatamente 2 (veja a Figura 3.17).

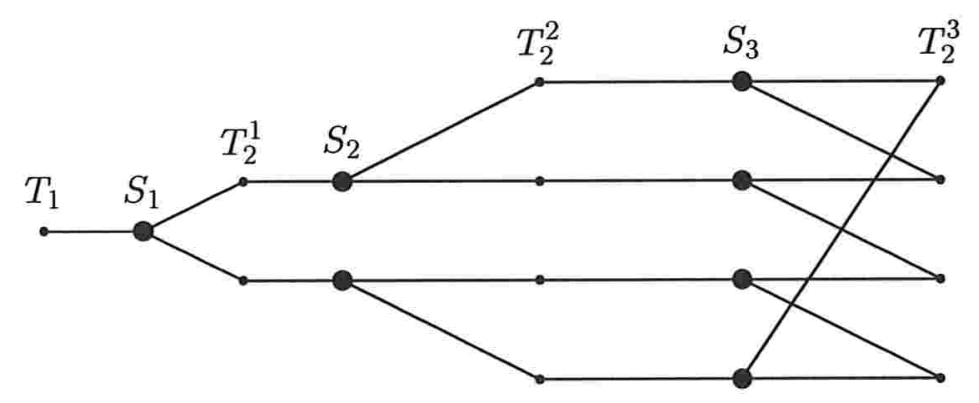

Figura 3.17: Exemplo do grafo $G \operatorname{com} r=3$.

Este grafo $G$ é o grafo de interseção de $\mathcal{T}_{G^{\prime}}$ (veja a Figura 3.18). 


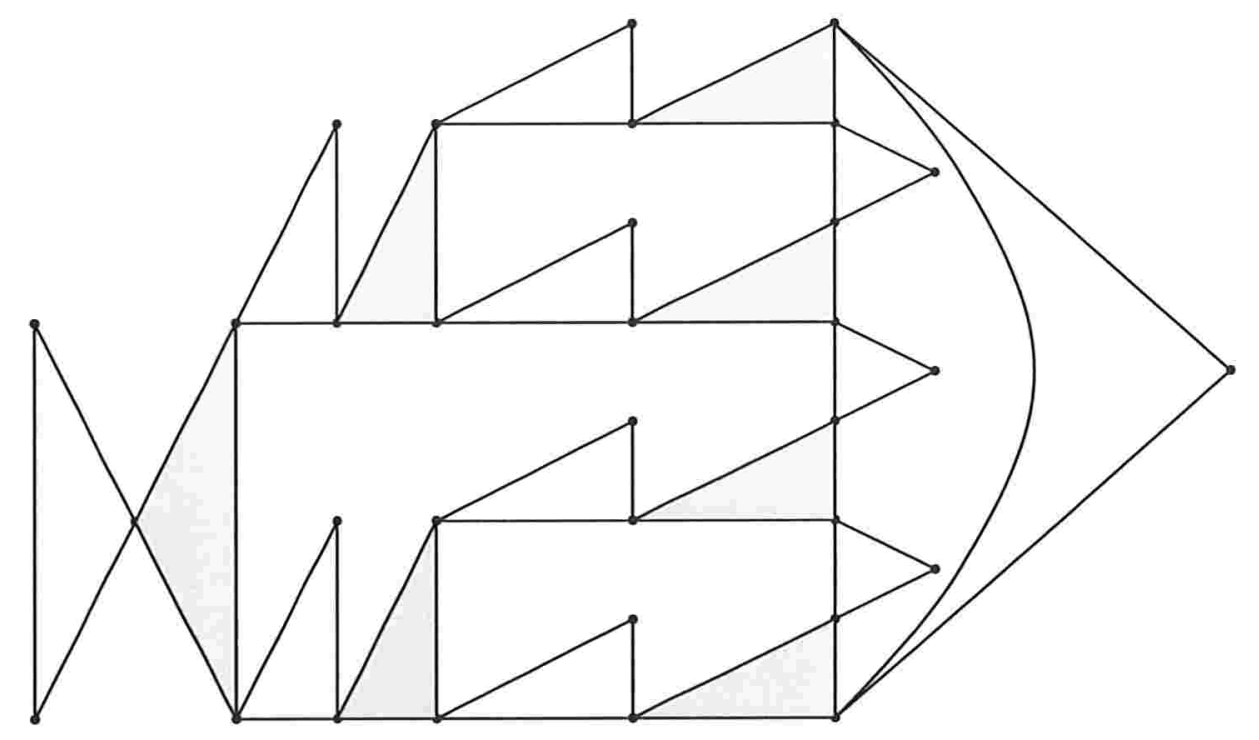

Figura 3.18: Exemplo de um grafo $G^{\prime} \operatorname{com} r=3$.

Note que o conjunto de triângulos de $G^{\prime}$ que corresponde ao conjunto de vértices $I=$ $\bigcup_{i=1}^{r} S_{i}$ de $G$ é $(r+1)$-ótimo em $G^{\prime}$. Isto é, para cada $p \leq r+1$, a união de quaisquer $p$ triângulos disjuntos nos vértices (dois a dois) em $G^{\prime}$ intersecta pelo menos $p$ triângulos que correspondem ao conjunto $I$ de $G$. No entanto, um empacotamento máximo de $\mathcal{T}_{V}\left(G^{\prime}\right)$ é o conjunto de triângulos que corresponde ao conjunto de vértices $I^{*}=T_{1} \cup\left(\bigcup_{i=1}^{r} T_{2}^{i}\right)$ de $G$. Observe que a razão $\left|I^{*}\right| /|I|$ é $1+\frac{2^{r-1}}{2^{r}-1}=\frac{3 \cdot 2^{r}-2}{2 \cdot 2^{r}-2}$. Portanto, a razão $\left|I^{*}\right| /|I|$ se aproxima de $3 / 2$ à medida que $r$ aumenta.

Observamos ainda que o exemplo justo para o problema do empacotamento máximo de $k$-conjuntos apresentado por Hurkens e Schrijver [23] dá origem a uma instância do VTP, isto é, um grafo $G$, para o qual a razão do valor de $\operatorname{opt}_{\mathrm{VTP}}(G)$ sobre o valor da solução obtida pelo algoritmo $\operatorname{HS}\left(\mathcal{T}_{V}(G), t\right)$ se aproxima de $3 / 2$ (quando $t$ cresce), e $G$ é irredundante com $\Delta(G)=4$. No entanto, tal grafo $G$ tem muito mais triângulos do que o exemplo de $\mathrm{Yu}$ e Goldschmidt [30] que apresentamos acima. Por exemplo, para $t=4$ esse grafo tem 285 vértices e 152 triângulos (95 numa solução ótima e 57 na solução obtida pelo algoritmo de busca local). 


\subsection{Empacotamento de triângulos disjuntos nos vértices em grafos de intervalos unitários}

\subsubsection{Introdução}

Um grafo de intervalos é um grafo de interseção de um conjunto finito de intervalos em $\mathbb{R}$. Quando todos esses intervalos têm o mesmo tamanho, temos um grafo de intervalos unitários. Quando nenhum dos intervalos contém qualquer outro, temos um grafo de intervalos próprios. Um grafo $G$ é um grafo de indiferença (indifference graph) se existe um número positivo $\delta$ e uma função $f: V_{G} \rightarrow \mathbb{R}$, tal que, para todo par $u, v \in V_{G}, u \neq v, u v$ é aresta de $G$ se e somente se $|f(u)-f(v)|<\delta$.

Roberts [28] mostrou que as classes de grafos de indiferença, grafos de intervalos unitários e grafos de intervalos próprios são equivalentes.

Descrevemos a seguir um algoritmo linear para o problema VTP restrito a grafos de intervalos unitários. Esse algoritmo é baseado na seguinte caracterização, devida a Looges e Olariu [26]: um grafo $G$ é um grafo de intervalos unitários se, e somente se, existe uma ordenação linear < de vértices de $G$ tal que, para cada escolha de vértices $u, v, w$ em $V_{G}$ temos que

$$
\text { se } u<v<w \text { e } u w \in E_{G} \text {, então } u v, v w \in E_{G} \text {. }
$$

Essa ordenação < será chamada canônica.

\subsubsection{Algoritmo exato}

Apresentamos nesta seção o algoritmo linear VTindifference para o problema VTP em grafos de intervalos unitários.

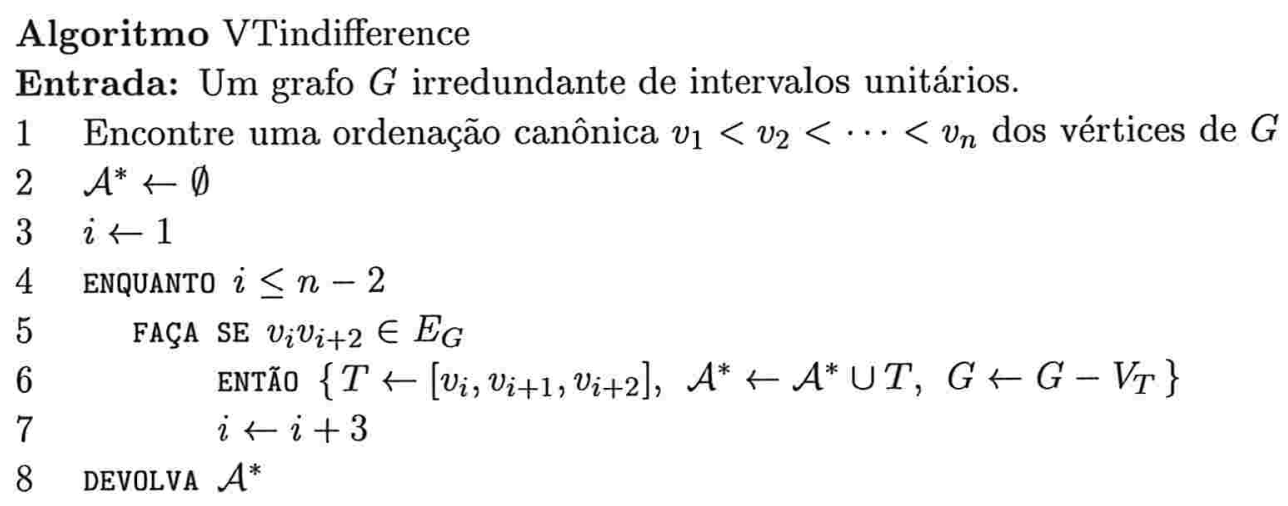

Teorema 3.16 $O$ algoritmo VTindifference aplicado a um grafo de intervalos unitários $G$ devolve um empacotamento máximo de $\mathcal{T}_{V}(G)$ em tempo linear.

Demonstração. Suponha que, para um grafo de intervalos unitários $G$, a coleção $\mathcal{A}^{*}$ devolvida pelo algoritmo não seja uma solução ótima. Sejam $\mathcal{T}^{*}$ um empacotamento máximo de $\mathcal{T}_{V}(G)$ que tem o número máximo de triângulos em comum com $\mathcal{A}^{*}$, e $i$ o menor número tal 
que $\left[v_{i}, v_{i+1}, v_{i+2}\right] \in \mathcal{A}^{*} \backslash \mathcal{T}^{*}$. Denotamos o triângulo $\left[v_{i}, v_{i+1}, v_{i+2}\right]$ por $T$. Vamos mostrar que

$$
\text { Se } T^{\prime} \in \mathcal{T}^{*}, T^{\prime} \cap T \neq \emptyset \text { e } v_{j} \in V_{T^{\prime}} \backslash V_{T}, \text { então } j>i+2 \text {. }
$$

Suponha, por contradição, que $j \leq i+2$. Como $v_{j} \notin V_{T}$, temos que $j<i$.

Se $v_{j}$ é um vértice de um triângulo $T^{\prime \prime} \mathrm{em} \mathcal{A}^{*}$, pela definição do número $i$ e pelo fato de que $j<i$, temos que $T^{\prime \prime} \in \mathcal{T}^{*} \cap \mathcal{A}^{*}$. Além disso, como $T \in \mathcal{A}^{*}$ e $T^{\prime} \cap T \neq \emptyset$ podemos ver que $T^{\prime} \notin \mathcal{A}^{*}$, e portanto $T^{\prime} \neq T^{\prime \prime}$. Notamos que nesse caso $v_{j}$ é coberto por $T^{\prime}$ e $T^{\prime \prime}$, ambos triângulos de $\mathcal{T}^{*}$, o que é impossível. Segue, portanto, que $v_{j}$ não é coberto por qualquer triângulo de $\mathcal{A}^{*}$. Analisamos dois possíveis casos.

(i) $v_{j} v_{i+1} \in E_{G}$ ou $v_{j} v_{i+2} \in E_{G}$. De $j<i$ e (3.31), segue que $v_{j} v_{j+2} \in E_{G}$. Como $v_{j}$ não está coberto por nenhum triângulo de $\mathcal{A}^{*}$, o algoritmo incluiria o triângulo $\left[v_{j}, v_{j+1}, v_{j+2}\right]$ na solução, uma contradição.

(ii) $v_{j} v_{i+1}, v_{j} v_{i+2} \notin E_{G}$. Nesse caso, é claro que $v_{i}, v_{j} \in V_{T^{\prime}}$. Seja $v_{k}$ o terceiro vértice de $T^{\prime}$. Note que $v_{k} \notin V_{T}$. Se $k \leq i+2$ então (assim como para $v_{j}$ ) segue que $k<i$ e que $v_{k}$ não está coberto por nenhum triângulo de $\mathcal{A}^{*}$. Para $l:=\min \{j, k\}, j, k<i, v_{l} v_{i} \in E_{G}$ e (3.31) implicam em $v_{l} v_{l+2} \in E_{G}$. Mas nesse caso, o algoritmo incluiria o triângulo $\left[v_{l}, v_{l+1}, v_{l+2}\right]$ na solução, uma contradição. Se $k>i+2$ então $j<i, v_{j} v_{k} \in E_{G}$ e (3.31) implicam em $v_{j} v_{j+2} \in E_{G}$, novamente uma contradição.

Provamos então a afirmação (3.32).

Se um único triângulo $T_{1}$ de $\mathcal{T}^{*}$ intersecta $T$, substituindo $T_{1}$ por $T$ obtemos um empacotamento máximo de $\mathcal{T}_{V}(G)$ que contém mais triângulos em comum com $\mathcal{A}^{*}$ do que $\mathcal{T}^{*}$, uma contradição.

Se exatamente dois triângulos $T_{1}$ e $T_{2}$, de $\mathcal{T}^{*}$ intersectam $T$, como $T_{1}$ e $T_{2}$ são disjuntos nos vértices existem pelo menos 3 vértices distintos, digamos $v_{k}, v_{l}$ e $v_{p}$, em $\left(V_{T_{1}} \cup V_{T_{2}}\right) \backslash V_{T}$. De (3.32) temos que $k, l, p>i+2$. Suponha, sem perda de generalidade, que $p=\max \{k, l, p\}$. Como $v_{p}$ é adjacente a pelo menos um dos vértices $v_{i}, v_{i+1}, v_{i+2}$, e $k, l, p>i+2$, por (3.31) temos que $\left[v_{k}, v_{l}, v_{p}\right]$ é um triângulo em $G$. Substituindo $T_{1}$ e $T_{2}$ por $T$ e $\left[v_{k}, v_{l}, v_{p}\right]$, obtemos uma solução ótima que tem mais triângulos em comum $\operatorname{com} \mathcal{A}^{*}$ do que $\mathcal{T}^{*}$, uma contradição.

Se três triângulos, $T_{1}, T_{2}$, e $T_{3}$ de $\mathcal{T}^{*}$ intersectam $T$, então existem 6 vértices distintos em $\left(V_{T_{1}} \cup V_{T_{2}} \cup V_{T_{3}}\right) \backslash V_{T}$. Por (3.32), sabemos que os índices de todos estes triângulos são maiores que $i+2$. Além disso, assim como fizemos acima, usando (3.31) concluímos que estes triângulos induzem um subgrafo completo de $G$. Portanto, podemos substituir $T_{1}, T_{2}$ e $T_{3}$ por $T$ e dois outros triangulos disjuntos nos vértices, cujos vértices estão em $\left(V_{T_{1}} \cup V_{T_{2}} \cup V_{T_{3}}\right) \backslash V_{T}$. Temos novamente uma contradição.

Como a ordenação canônica pode ser computada em tempo linear [26] e como a iteração das linhas 3 - 5 claramente consomem tempo linear, segue que o algoritmo VTindifference é linear.

\subsubsection{Observações finais}

Investigamos o problema VTP para a classe dos grafos de intervalos. Não conseguimos encontrar um algoritmo polinomial para este caso, e nem tampouco mostrar que este caso é 
NP-difícil. Observamos que um algoritmo guloso simples não resolve o VTP para essa classe de grafos. Na Figura 3.19 exibimos um grafo de intervalos (não-unitários) para o qual uma solução gulosa não é uma solução ótima. Técnicas como programação dinâmica, que aparentam ser apropriadas para este caso, não nos conduziram a resultados positivos. Seria interessante obter um resultado para este caso. Lembramos que, para a classe dos grafos cordais (que contém a classe dos grafos intervalos), o problema VTP é NP-difícil, conforme mencionamos no início deste capítulo.

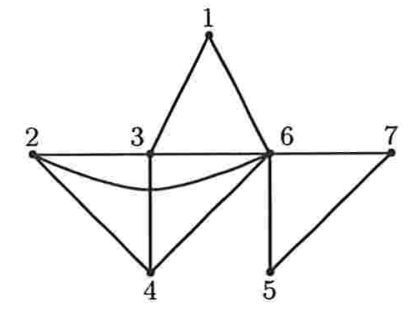

(a)

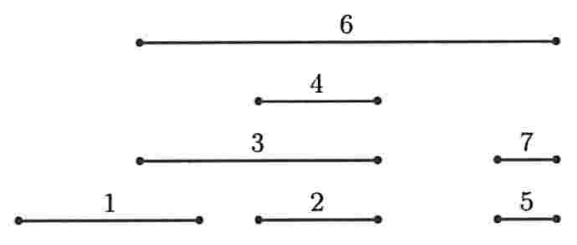

(b)

Figura 3.19: (a) Um grafo de intervalos, que é o grafo de interseção do conjunto de intervalos mostrados em (b). Um algoritmo guloso pode devolver o triângulo $[1,3,6]$. Uma solução ótima é a coleção $\{[2,3,4],[5,6,7]\}$.

Uma observação interessante é que o algoritmo VTindifference pode ser generalizado de modo que, dado um grafo de intervalos unitários $G$ e um número inteiro fixo $r \geq 2$, ele encontra em $G$, em tempo linear, o número máximo de cliques de ordem $r$, duas a duas disjuntas nos vértices. 


\section{Capítulo 4}

\section{Empacotamento de triângulos disjuntos nas arestas}

\subsection{Introdução}

Neste capítulo trataremos do problema do empacotamento máximo de triângulos disjuntos nas arestas, denotado por ETP. Holyer [21] mostrou que ETP é NP-difícil. Lembramos que ETP- $k$ denota o problema ETP restrito a grafos de grau máximo $k$.

Em 2002, Caprara e Rizzi [7] exibiram um algoritmo polinomial para o problema ETP4, e mostraram que ETP-5 é APX-difícil (isto é, não admite um esquema de aproximação polinomial a menos que $\mathrm{P}=\mathrm{NP}$ ).

Apresentamos neste capítulo um algoritmo de aproximação para o problema ETP-5 cuja razão de aproximação é $\frac{4}{3}$.

Neste capítulo, restringimos nossa atenção aos grafos irredundantes. Quando escrevemos $G-U$ (para $U \subseteq V_{G}$ ou $U \subseteq E_{G}$ ) assumimos que os vértices isolados e as arestas que não pertencem a nenhum triângulo foram removidos também.

\subsection{Grafos arbitrários}

Tal como ocorre no caso do problema VTP, um algoritmo guloso simples que retorna uma coleção maximal de triângulos disjuntos nas arestas é uma 3-aproximação para o problema ETP. Isto segue do fato de que todo triângulo de uma solução obtida pelo algoritmo guloso intersecta no máximo 3 triângulos de uma solução ótima.

Observamos também que o algoritmo $\operatorname{HS}(\mathcal{T}, t)$ de Hurkens e Schrijver [23] para o problema do empacotamento máximo de $k$-conjuntos (mencionado na seção 3.2.1) nos fornece uma $\left(\frac{3}{2}+\varepsilon\right.$ )-aproximação para o ETP, onde $\varepsilon$ é inversamente proporcional a $t$ (conforme $t$ aumenta, $\varepsilon$ tende a zero). Analogamente ao caso do VTP, basta tomar $k=3$ e $\mathcal{T}=\mathcal{T}_{E}(G)$, onde $\mathcal{T}_{E}(G)$ denota a coleção dos conjuntos das arestas de todos os triângulos em $G$. Eles exibiram ainda, para cada $t$, instâncias para as quais o algoritmo acima produz soluções que atingem tal razão em relação ao empacotamento máximo de triângulos disjuntos nas arestas. A razão de aproximação obtida por Hurkens e Schrijver é a melhor razão de aproximação que se conhece para o ETP (para grafos arbitrários). 


\subsection{Grafos com grau máximo 5}

Nesta seção, restringimos nossa atenção aos grafos irredundantes de grau máximo 5 .

Considerando que, em termos de grau, instâncias com grau máximo 5 para o problema ETP constituem um caso difícil, que está no limite entre instâncias difíceis e fáceis [7], investigamos o ETP-5 com o objetivo de encontrar um algoritmo com uma razão de aproximação melhor que 3/2. Desenvolvemos um algoritmo de aproximação para o ETP-5 cuja razão de aproximação é $\frac{4}{3}$. Esse algoritmo será apresentado a seguir.

\subsubsection{Algoritmo de aproximação ET5}

Chamaremos de $\operatorname{ET} 5(G)$ o algoritmo de aproximação que desenvolvemos para o problema ETP-5. Neste algoritmo tem um papel fundamental o grafo de Hajós, denotado por $H:=$ $H\left[T_{1}, T_{2}, T_{3}\right]$. O grafo de Hajós consiste em um circuito de tamanho 6 e três cordas que induzem um triângulo; os outros três triângulos (cada um contendo uma destas cordas) são denotados por $T_{1}, T_{2}$ e $T_{3}$. Veja a Figura 4.1 .

O algoritmo $\operatorname{ET} 5(G)$ verifica se existe um grafo de Hajós $H=H\left[T_{1}, T_{2}, T_{3}\right]$ em $G$. Se existe, ele adiciona os triângulos $T_{1}, T_{2}$ e $T_{3}$ ao conjunto $\mathcal{A}^{*}$ a ser devolvido pelo algoritmo, retira $E_{H}$ do grafo $G$ e repete o processo. Caso contrário, ele adiciona $\left\{T: E_{T} \in \operatorname{HS}\left(\mathcal{T}_{E}(G), 3\right)\right\}$ ao conjunto $\mathcal{A}^{*}$ (onde HS é o algoritmo baseado em busca local definido na seção 3.2 .1 ).
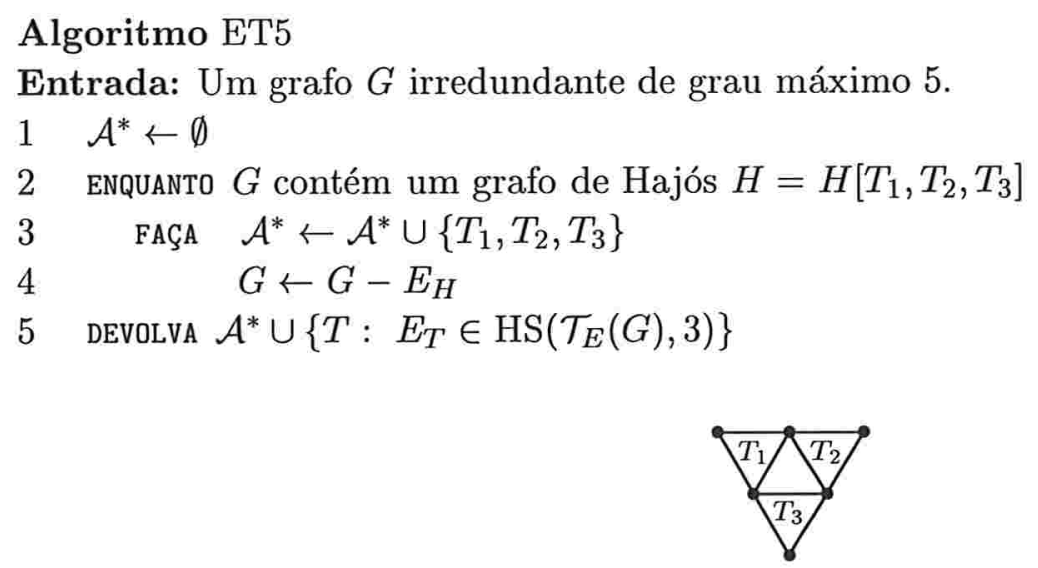

Figura 4.1: O grafo de Hajós denotado por $H\left[T_{1}, T_{2}, T_{3}\right]$.

\subsubsection{Desempenho do algoritmo ET5}

Para obter a razão de aproximação do algoritmo ET5, precisamos dos seguintes lemas.

Lema 4.1 Se G é um grafo que contém um grafo de Hajós $H$, então o número de triângulos que têm alguma aresta em comum com $H$, em qualquer empacotamento máximo de $\mathcal{T}_{E}(G)$, é no máximo 4.

A prova do lema acima pode ser obtida facilmente por inspeção.

Lema 4.2 Se $G$ é um grafo que não contém nenhum grafo de Hajós, o algoritmo $\operatorname{HS}\left(\mathcal{T}_{E}(G), 3\right)$ é uma $\frac{4}{3}$-aproximação para o problema ETP-5. 
Demonstração. A prova é por indução no número de triângulos em $G$. Para $\left|\mathcal{T}_{G}\right|=0$ e $\left|\mathcal{T}_{G}\right|=1$, a prova do lema é imediata. Sejam $G$ um grafo com $\left|\mathcal{T}_{G}\right|>1, \mathcal{T}^{*}$ um empacotamento máximo de $\mathcal{T}_{E}(G)$, e $\mathcal{A}^{*}:=\left\{T: E_{T} \in \operatorname{HS}\left(\mathcal{T}_{E}(G), 3\right)\right\}$. Mostramos em seguida que existe uma coleção de triângulos $\mathcal{A}$ tal que

$\mathcal{A} \subseteq \mathcal{A}^{*},|\mathcal{A}| \leq 3$ e $\frac{|\mathcal{T}|}{|\mathcal{A}|} \leq \frac{4}{3}$, onde $\mathcal{T}$ é um conjunto de triângulos de $\mathcal{T}^{*}$ que têm pelo menos uma aresta em comum com algum triângulo em $\mathcal{A}$.

Para isso, analisamos quatro casos.

(i) Se existe um triângulo $T \in \mathcal{A}^{*} \cap \mathcal{T}^{*}$, então definimos $\mathcal{A}:=\{T\}$.

(ii) Se o caso (i) não é satisfeito e $\mathcal{A}^{*}$ contém triângulos distintos $T_{1}, T_{2}, T_{3}$ tais que $\mid V_{T_{1}} \cap$ $V_{T_{2}}|=| V_{T_{2}} \cap V_{T_{3}} \mid=1$, então definimos $\mathcal{A}:=\left\{T_{1}, T_{2}, T_{3}\right\}$ (veja Figura 4.2). Usando o fato de que $G$ não contém nenhum grafo de Hajós e que o grau máximo de $G$ é 5 , é fácil ver que o número de triângulos de $\mathcal{T}^{*}$ que têm pelo menos uma aresta em comum com algum triângulo em $\mathcal{A}$ é no máximo 4 .

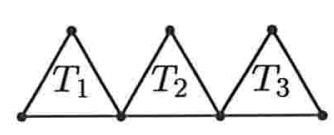

Figura 4.2: Triângulos $T_{1}, T_{2}$ e $T_{3}$ do caso (ii).

(iii) Se os casos (i) e (ii) não são satisfeitos e $\mathcal{A}^{*}$ contém triângulos distintos $T_{1}$ e $T_{2}$ tais que $\left|V_{T_{1}} \cap V_{T_{2}}\right|=1$, então definimos $\mathcal{A}:=\left\{T_{1}, T_{2}\right\}$. Suponha que $|\mathcal{T}| \geq 3$. Se nenhum triângulo de $\mathcal{A}^{*}$, exceto $T_{1}$ e $T_{2}$, tem pelo menos uma aresta em comum com algum triângulo em $\mathcal{T}$, então $T_{1}$ e $T_{2}$ poderiam ser substituídos por $\mathcal{T}$, uma contradição. Portanto, existe um triângulo $T_{3}\left(T_{3} \neq T_{1}, T_{2}\right)$ em $\mathcal{A}^{*}$ que tem uma aresta em comum com algum triângulo de $\mathcal{T}$. Como $G$ não contém o grafo de Hajós, temos que os triângulos $T_{1}, T_{2}$ e $T_{3}$ satisfazem a condição do caso (ii), o que é de novo uma contradição. Concluímos, portanto, que $|\mathcal{T}| \leq 2$.

(iv) Se os casos (i), (ii) e (iii) não são satisfeitos, então seja $T_{1}$ um triângulo qualquer de $\mathcal{A}^{*}$ e $\mathcal{A}:=\left\{T_{1}\right\}$. Suponha que $|\mathcal{T}| \geq 2$. Se o único triângulo de $\mathcal{A}^{*}$ que tem uma aresta em comum com algum triângulo em $\mathcal{T}$ é $T_{1}$, então $T_{1}$ poderia ser substituído por triângulos em $\mathcal{T}$, uma contradição. Portanto, existe um triângulo $T_{2}\left(T_{2} \neq T_{1}\right)$ em $\mathcal{A}^{*}$ que tem uma aresta em comum com algum triângulo de $\mathcal{T}$, ou seja, temos o caso (iii), uma contradição. Concluímos portanto que $|\mathcal{T}| \leq 1$.

Em todos os casos acima temos que a afirmação (4.1) é válida.

Seja $\mathcal{A}$ uma coleção que satisfaz (4.1) e $G^{\prime}:=G-\bigcup_{T \in \mathcal{A}} E_{T}$. Observamos que $\mathcal{A}^{*} \backslash \mathcal{A}$ é 3-ótimo em $G^{\prime}$ (caso contrário, $\mathcal{A}^{*}$ não seria 3-ótimo em $G$ ). Portanto, pela hipótese da indução temos que opt $\left(G^{\prime}\right) \leq \frac{4}{3}\left|\mathcal{A}^{*} \backslash \mathcal{A}\right|$. Logo,

$$
\operatorname{opt}(G) \leq \operatorname{opt}\left(G^{\prime}\right)+|\mathcal{T}| \leq \frac{4}{3}\left|\mathcal{A}^{*} \backslash \mathcal{A}\right|+|\mathcal{T}|=\frac{4}{3}\left|\mathcal{A}^{*}\right|-\frac{4}{3}|\mathcal{A}|+|\mathcal{T}|
$$


Como $\frac{|\mathcal{T}|}{|\mathcal{A}|} \leq \frac{4}{3}$, das desigualdades acima segue que opt $(G) \leq \frac{4}{3}\left|\mathcal{A}^{*}\right|$

Teorema 4.3 O algoritmo ET5 é uma $\frac{4}{3}$-aproximação para o problema ETP-5.

Demonstração. Seja $G^{\prime}$ o grafo $G$ imediatamente antes da execução da linha 5 do algoritmo ET5 e $j$ o número de iterações (linhas $2-4$ ) feitas pelo algoritmo. Pelo Lema 4.1, opt $\left(G^{\prime}\right) \geq$ $\operatorname{opt}(G)-4 j$. Se $G^{\prime} \neq \emptyset$, então claramente, $G^{\prime}$ não contém nenhum grafo de Hajós. Logo, pelo Lema 4.2, $\operatorname{opt}_{\mathrm{ETP}}\left(G^{\prime}\right) \leq \frac{4}{3}\left|\left\{T: E_{T} \in \operatorname{HS}\left(\mathcal{T}_{E}\left(G^{\prime}\right), 3\right)\right\}\right|$. Além disso, $\left|\mathcal{A}^{*}\right|=3 j$ (onde $\mathcal{A}^{*}$ é o conjunto na linha 5 do algoritmo). Portanto,

$$
\begin{aligned}
\operatorname{opt}_{\mathrm{ETP}}(G) & \leq 4 j+\operatorname{opt}_{\mathrm{ETP}}\left(G^{\prime}\right) \leq 4 \frac{\left|\mathcal{A}^{*}\right|}{3}+\frac{4}{3}\left|\left\{T: E_{T} \in \operatorname{HS}\left(\mathcal{T}_{E}\left(G^{\prime}\right), 3\right)\right\}\right| \\
& =\frac{4}{3} \mid \mathcal{A}^{*} \cup\left\{T: E_{T} \in \operatorname{HS}\left(\mathcal{T}_{E}\left(G^{\prime}\right), 3\right)\right\} .
\end{aligned}
$$

Proposição 4.4 A razão de aproximação $\frac{4}{3}$ do algoritmo ET5 é justa.

Demonstração. Considere o grafo da Figura 4.3.

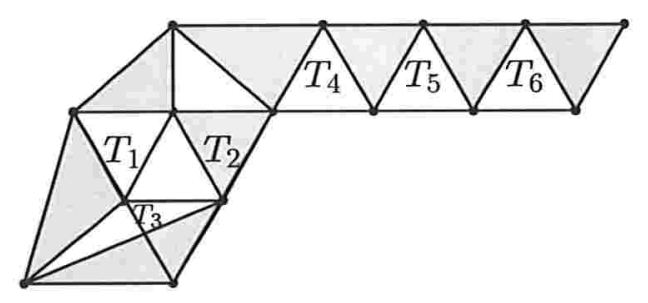

Figura 4.3: Os 8 triângulos destacados compõem uma solução ótima de ETP. O algoritmo ET5 pode devolver os triângulos $T_{1}, \ldots, T_{6}$ (se começar removendo $H=H\left[T_{1}, T_{2}, T_{3}\right]$ ).

\subsubsection{Complexidade computacional do algoritmo ET5}

Não é difícil ver que o algoritmo ET5 consome tempo $O\left(n_{G}^{3}\right)$, onde $n_{G}$ é a ordem do grafo de entrada. De fato, as linhas $2-4$ podem ser executadas em tempo $O\left(\left|\mathcal{T}_{G}\right|^{2}\right)$, pois em cada iteração, verifica-se para cada triângulo se ele é o centro de um grafo de Hajós. Ainda, o número de iterações é limitado por $\left|\mathcal{T}_{G}\right| / 4$. Além disso, o conjunto $\left\{T: E_{T} \in \operatorname{HS}\left(\mathcal{T}_{E}(G), 3\right)\right\}$ pode ser encontrado em tempo $O\left(\left|\mathcal{T}_{G}\right|^{3}\right)$, pois em cada iteração do algoritmo $\operatorname{HS}\left(\mathcal{T}_{E}(G), 3\right)$, por meio de uma busca exaustiva, tentamos trocar 2 triângulos aresta-disjuntos da coleção atual por 3 triângulos aresta-disjuntos. Para isto, consumimos tempo $O\left(\left|\mathcal{T}_{G}\right|^{2}\right)$; ainda, o algoritmo $\operatorname{HS}\left(\mathcal{T}_{E}(G), 3\right)$ tem no máximo $\left|\mathcal{T}_{G}\right|$ iterações. Finalmente, como $\Delta(G)=5$, do Lema 3.1 temos que $\left|\mathcal{T}_{G}\right| \leq 4 n_{G}$. Logo, o consumo de tempo do algoritmo ET5 é $O\left(n_{G}^{3}\right)$. 


\section{Capítulo 5}

\section{Empacotamento de arestas e triângulos}

\subsection{Introdução}

$\mathrm{O}$ problema do $K_{2}+K_{3}$-empacotamento é o problema do $\mathcal{F}$-empacotamento em que $\mathcal{F}=$ $\left\{K_{2}, K_{3}\right\}$, e o objetivo é encontrar um grafo $H \subseteq G$ que seja uma união de grafos disjuntos nos vértices, sendo cada um deles isomorfo a um grafo de $\mathcal{F}$, tal que $H$ tenha o maior número possível de arestas. Observamos que se o objetivo é maximizar o número de vértices de $H$, então o problema correspondente pode ser resolvido em tempo polinomial [19]. Já o problema de nosso interesse é NP-difícil. Para verificar isso, pode-se fazer uma redução a partir do problema do empacotamento de triângulos (como fizemos na prova do Teorema 5.1).

Neste capítulo mostramos que o $K_{2}+K_{3}$-empacotamento é APX-completo em grafos com grau máximo 5. Para isso, mostramos uma L-redução do problema VTP em grafos de grau máximo 4. Mostramos também que o $\mathrm{K}_{2}+\mathrm{K}_{3}$-empacotamento é APX-completo mesmo em grafos de grau máximo 4 tais que toda aresta pertence a algum triângulo. Utilizamos uma L-redução do problema MAX2SAT3. Ela é mais elaborada, e é baseada na idéia de Caprara e Rizzi [7] para mostrar que o problema VTP em grafos de grau máximo 4 é APX-completo.

Definimos o problema MAX2SAT3 como segue. Dada uma coleção de cláusulas $C=$ $\left\{c_{1}, c_{2}, \ldots, c_{l}\right\}$ sobre um conjunto finito $X=\left\{x_{1}, x_{2}, \ldots, x_{p}\right\}$ de variáveis, com $\left|c_{i}\right|=2$ para $1 \leq i \leq l$, e tal que toda variável aparece em no máximo três cláusulas (contando-se ambas as ocorrências positivas e negativas), encontre uma atribuição às variáveis de $X$ que satisfaz o maior número possível de cláusulas. Sabe-se que o problema MAX2SAT3 é APX-completo [4].

Ainda, mostramos uma $\left(1+\frac{1}{3} \rho\right)$-aproximação para o $\mathrm{K}_{2}+\mathrm{K}_{3}$-empacotamento, onde $\rho$ é uma razão de aproximação para o problema VTP. Nosso algoritmo inicialmente aplica uma rotina para encontrar o maior número possível de triângulos disjuntos nos vértices, e depois complementa a solução com um emparelhamento máximo no grafo obtido removendo-se os vértices dos triangulos que a rotina encontrou. Usando como rotina o algoritmo de Hurkens e Schrijver [23] para o VTP, mostramos que a razão de aproximação do nosso algoritmo é $\frac{3}{2}+\varepsilon$. Ressaltamos ainda que o algoritmo de aproximação para o VTP-4 que obtivemos (com razão um pouco menor que 1,2 ) resulta em um algoritmo de aproximação com razão 1,4 para o problema do $K_{2}+K_{3}$-empacotamento em grafos de grau máximo 4 . 
No final deste capítulo, apresentamos uma 3/2-aproximação para o problema do $\mathrm{K}_{2}+$ $\mathrm{K}_{3}$-empacotamento, sugerida recentemente por Raphael Yuster. Também comentamos que um resultado obtido por Halldórsson [16] para cobertura por 3-conjuntos fornece uma 3/2aproximação para esse problema.

\subsection{Resultados de inaproximabilidade}

Teorema 5.1 O problema do $\mathrm{K}_{2}+\mathrm{K}_{3}$-empacotamento é APX-completo em grafos de grau máximo 5.

Demonstração. Exibiremos uma L-redução do problema VTP-4 em grafos irredundantes que é APX-completo [7] ao problema do $\mathrm{K}_{2}+\mathrm{K}_{3}$-empacotamento. Dado um grafo irredundante $G$ com $\Delta(G)=4$, definimos $G^{\prime}:=f(G)$ como a união de duas cópias $G_{1}$ e $G_{2}$ de $G$, junto com o conjunto de arestas $\left\{u_{1} u_{2}: u_{1} \in V_{G_{1}}, u_{2} \in V_{G_{2}}\right.$, e $u_{1}, u_{2}$ correspondem a um mesmo vértice $u \in$ $\left.V_{G}\right\}$ (veja a Figura 5.1).

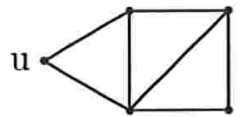

G

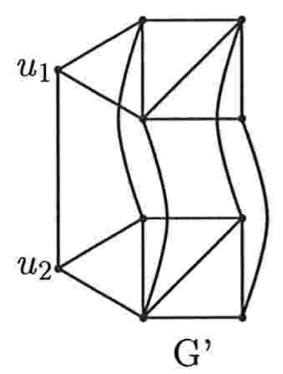

Figura 5.1: Uma L-redução do problema VTP-4 em grafos irredundantes ao problema do $\mathrm{K}_{2}+\mathrm{K}_{3}$ empacotamento.

Inicialmente mostramos que

$$
\operatorname{opt}_{\mathrm{K}_{2}+\mathrm{K}_{3}}\left(G^{\prime}\right)=3 \operatorname{opt}_{\mathrm{VTP}}(G)+n_{G} \text {. }
$$

Se $\mathcal{T}^{*}$ é uma solução ótima de VTP em $G$, então um empacotamento de $\left\{K_{2}, K_{3}\right\}$ em $G^{\prime}$ consiste em triângulos de $G_{1}$ e $G_{2}$ que são cópias de triângulos em $\mathcal{T}^{*}$, e o conjunto de arestas $\left\{u_{1} u_{2}: u_{1} \in V_{G_{1}}, u_{2} \in V_{G_{2}}\right.$, e $u_{1}, u_{2}$ correspondem a um mesmo vértice $u$ de $G$ não coberto por $\left.\mathcal{T}^{*}\right\}$. Como o número de vértices de $G$ não cobertos por $\mathcal{T}^{*}$ é $n_{G}-3 \operatorname{opt}_{\mathrm{VTP}}(G)$, temos $\operatorname{opt}_{K_{2}+K_{3}}\left(G^{\prime}\right) \geq 3 \operatorname{opt}_{\mathrm{VTP}}(G)+n_{G}$. Por outro lado, se uma solução ótima de $\mathrm{K}_{2}+\mathrm{K}_{3^{-}}$ empacotamento em $G^{\prime}$ tem $t$ triângulos e $e$ arestas, como $e \leq \frac{n_{G^{\prime}}-3 t}{2}=n_{G}-\frac{3}{2} t$, temos que $\operatorname{opt}_{\mathrm{K}_{2}+\mathrm{K}_{3}}\left(G^{\prime}\right)=3 t+e \leq \frac{3}{2} t+n_{G}$. Claramente, $t \leq 2 \operatorname{opt}_{\mathrm{VTP}}(G)$, e portanto opt $\mathrm{K}_{2}+\mathrm{K}_{3}\left(G^{\prime}\right) \leq$ $3 \operatorname{opt}_{\mathrm{VTP}}(G)+n_{G}$. Logo, a afirmação (5.1) é verdadeira.

Seja $\mathcal{T}^{*}$ uma solução ótima de VTP em $G$. Suponha que exista um triângulo $T \in \mathcal{T}^{*}$, tal que $T$ tenha 5 vértices vizinhos em $V_{G} \backslash V_{T}$ que não são cobertos por $\mathcal{T}^{*}$. Como $\Delta(G)=4$, um par deles, digamos $v_{1}, v_{2}$, é adjacente a um mesmo vértice, digamos $x$, de $V_{T}$; outro par, digamos $v_{3}, v_{4}$, é adjacente a um mesmo vértice, digamos $y$, de $V_{T}$. Note que o terceiro vértice de $V_{T}$, digamos $z$, tem grau pelo menos 3. Além disso, como $G$ é irredundante e $\Delta(G)=4$, temos que $v_{1} v_{2}, v_{3} v_{4} \in E_{G}$. De fato, como $G$ é irredundante, a aresta $v_{1} x$ (resp. $v_{2} x$ ) é aresta de algum triângulo. Como $d_{G}(x)=d_{G}(y)=\Delta(G)=4$, o único triângulo possível que tem a 
aresta $x v_{1}$ mas não tem a aresta $v_{1} v_{2}$, é o triângulo $\left[x, v_{1}, z\right]$ (veja a Figura 5.2). Mas agora, o único triângulo possível que tem a aresta $x v_{2}$ é o triângulo $\left[x, v_{2}, v_{1}\right]$, e portanto, $v_{1} v_{2} \in E_{G}$. Similarmente, $v_{3} v_{4} \in E_{G}$.

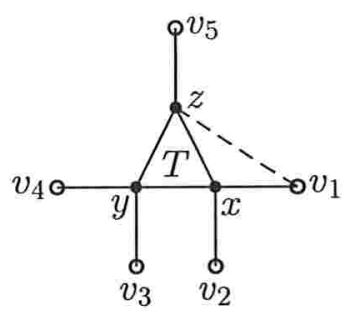

Figura 5.2: Os vértices $v_{1}, v_{2}, v_{3}, v_{4}$ e $v_{5}$ estão na vizinhança de $V_{T}$, e não são cobertos por $\mathcal{T}^{*}$.

Logo, substituindo $T$ por $\left[x, v_{1}, v_{2}\right]$ e $\left[y, v_{3}, v_{4}\right]$, obtemos uma solução para VTP que tem mais triângulos do que $\mathcal{T}^{*}$, uma contradição. Portanto, todo triângulo de $\mathcal{T}^{*}$ tem no máximo 4 vizinhos não cobertos por $\mathcal{T}^{*}$. Ainda, como $G$ é irredundante, todo vértice não coberto por $\mathcal{T}^{*}$ é adjacente a pelo menos um vértice coberto por $\mathcal{T}^{*}$. De fato, suponha que exista um vértice $v$ não coberto por $\mathcal{T}^{*}$, e não adjacente a nenhum triângulo coberto por $\mathcal{T}^{*}$. Como $G$ é irredundante, $v$ é vértice de um triângulo $T$. Observe que nenhum vértice de $T$ é coberto por $\mathcal{T}^{*}$, e portanto, $\mathcal{T}^{*}$ não é solução ótima de VTP em $G$, uma contradição. Segue, então, que o número de vértices em $G$ não cobertos por $\mathcal{T}^{*}$ é no máximo $4 \operatorname{opt}_{\mathrm{VTP}}(G)$, ou seja, $n_{G}-3 \operatorname{opt}_{\mathrm{VTP}}(G) \leq 4 \operatorname{opt}_{\mathrm{VTP}}(G)$. Usando (5.1), temos $\operatorname{opt}_{\mathrm{K}_{2}+\mathrm{K}_{3}}\left(G^{\prime}\right) \leq 10 \operatorname{opt}_{\mathrm{VTP}}(G)$, isto é, $\alpha=10$.

Se $\mathcal{A}$ é um empacotamento de $\left\{K_{2}, K_{3}\right\}$ em $G^{\prime}$, definimos $g(G, \mathcal{A})$ como o maior dos dois conjuntos $\mathcal{T}_{\mathcal{A}} \cap G_{1}, \mathcal{T}_{\mathcal{A}} \cap G_{2}$. Suponha, sem perda de generalidade, que $g(G, \mathcal{A})=\mathcal{T}_{\mathcal{A}} \cap G_{1}$. Seja $t_{1}:=\left|\mathcal{T}_{\mathcal{A}} \cap G_{1}\right|, t_{2}:=\left|\mathcal{T}_{\mathcal{A}} \cap G_{2}\right|, e_{1}:=\left|\mathcal{E}_{\mathcal{A}} \cap G_{1}\right|, e_{2}:=\left|\mathcal{E}_{\mathcal{A}} \cap G_{2}\right|$, e seja $e$ o número de arestas em $\mathcal{A}$ com um extremo em $G_{1}$ e outro em $G_{2}$. Claramente, $t_{1} \leq \operatorname{opt}_{\mathrm{VTP}}(G)$. Logo, $\frac{1}{2} t_{1}+\frac{3}{2} t_{1}-2$ opt $_{\mathrm{VTP}}(G) \leq 0$. Como $t_{2} \leq t_{1}$, temos que $\frac{1}{2} t_{1}+\frac{3}{2} t_{2}-2$ opt $_{\mathrm{VTP}}(G) \leq 0$, ou equivalentemente,

$$
\mathrm{opt}_{\mathrm{VTP}}(G)-t_{1} \leq 3 \mathrm{opt}_{\mathrm{VTP}}(G)+\left(\frac{3}{2} t_{1}+\frac{3}{2} t_{2}+e_{1}+e_{2}+e\right)-\left(3 t_{1}+3 t_{2}+e_{1}+e_{2}+e\right) .
$$

Temos que $3 t_{1}+3 t_{2}+2 e_{1}+2 e_{2}+2 e \leq n_{G^{\prime}}=2 n_{G}$, e portanto,

$$
\frac{3}{2} t_{1}+\frac{3}{2} t_{2}+e_{1}+e_{2}+e \leq n_{G} \text {. }
$$

De (5.2) e (5.3) segue

$$
\operatorname{opt}_{\mathrm{VTP}}(G)-t_{1} \leq 3 \operatorname{opt}_{\mathrm{VTP}}(G)+n_{G}-\left(3 t_{1}+3 t_{2}+e_{1}+e_{2}+e\right) .
$$

Agora, usando (5.1), obtemos opt $\mathrm{VTP}_{\mathrm{VT}}(G)-t_{1} \leq \mathrm{opt}_{\mathrm{K}_{2}+\mathrm{K}_{3}}\left(G^{\prime}\right)-\operatorname{val}_{\mathrm{K}_{2}+\mathrm{K}_{3}}(f(G), \mathcal{A})$, ou seja, $\beta=1$. Como o problema $\mathrm{K}_{2}+\mathrm{K}_{3}$-empacotamento pertence à classe APX (veremos na seção 5.3 um algoritmo de aproximação para este problema), segue que ele é APX-completo.

Teorema $5.2 O$ problema do $K_{2}+K_{3}$-empacotamento é APX-completo, mesmo em grafos irredundantes de grau máximo 4. 
Demonstração. Mostramos uma L-redução a partir do problema MAX2SAT3. Seja $\varphi=$ $(C, X), C=\left\{c_{1}, c_{2}, \ldots, c_{l}\right\}$ e $X=\left\{x_{1}, x_{2}, \ldots, x_{p}\right\}$, uma instância de MAX2SAT3. Seja $m_{i}$ o número de ocorrências de $x_{i}$. Podemos assumir, sem perda de generalidade, que $m_{i} \geq 2$ (pois se $x_{i}$ aparece somente em uma cláusula, podemos atribuir a $x_{i}$ o valor que satisfaz tal cláusula). Definimos $G^{\prime}:=f(\varphi)$ como segue.

A cada cláusula $c_{j}$ associamos uma componente de teste $\mathcal{C}_{j}$. A componente de teste de uma cláusula com dois literais consiste de 4 triângulos $\left[t_{j}^{1}, s_{j}^{1}, r_{j}^{1}\right],\left[s_{j}^{1}, r_{j}^{1}, r_{j}^{2}\right],\left[s_{j}^{2}, r_{j}^{1}, r_{j}^{2}\right],\left[s_{j}^{1}, r_{j}^{2}, t_{j}^{2}\right]$ (mostrados na Figura 5.3(a)), enquanto a componente de teste associada a uma cláusula com um literal consiste de 3 triângulos $\left[t_{j}^{1}, s_{j}^{1}, r_{j}^{1}\right],\left[s_{j}^{1}, r_{j}^{1}, r_{j}^{2}\right],\left[s_{j}^{2}, r_{j}^{1}, r_{j}^{2}\right]$ (Figura 5.3(b)).

A cada variável $x_{i}$ associamos uma componente de atribuiçãa $\mathcal{X}_{i}$, conforme ilustramos na Figura 5.3(c). Esta componente consiste de $2 m_{i}$ triângulos $T_{1}, \ldots, T_{2 m_{i}}$, onde $T_{2 k-1}=$ $\left[a_{i}^{k}, v_{i}^{k-1}, u_{i}^{k}\right]$ e $T_{2 k}=\left[b_{i}^{k}, u_{i}^{k}, v_{i}^{k}\right], k=1, \ldots, m_{i}$ (os índices superiores são modulo $m_{i}$ ). A paridade de $T_{k}$ é a paridade de $k$.

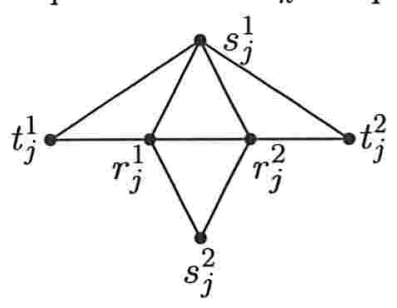

(a)

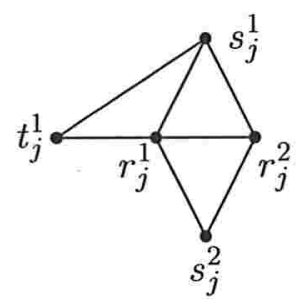

(b)

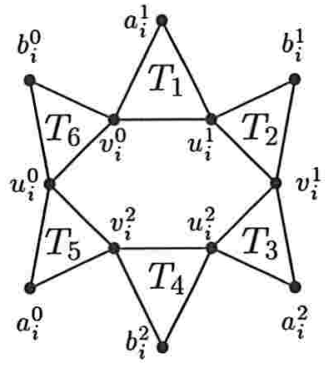

(c)

Figura 5.3: (a) Componente de teste de uma cláusula $c_{j}$ com dois literais. (b) Componente de teste de uma cláusula $c_{j}$ com um literal. (c) Componente de atribuição da variável $x_{i} \operatorname{com} m_{i}=3$.

O grafo $G^{\prime}$ é obtido conectando-se as componentes de testes e as componentes de atribuição como segue. Seja $c_{j}$ uma cláusula com dois literais e sejam $x_{1}, x_{2}$ as variáveis que ocorrem em $c_{j}$. Se $x_{i}$ ocorre positivo (resp. negado) em $c_{j}$, então identificamos o vértice $t_{j}^{i}$ da componente de teste $\mathcal{C}_{j}$ com um vértice $a_{i}^{k}$ (resp. $b_{i}^{k}$ ) da componente de atribuição $\mathcal{X}_{i}$ que ainda não foi envolvida em nenhuma identificação. Similarmente, seja $c_{j}$ uma cláusula com somente um literal, digamos, $x_{1}$. Se $x_{1}$ ocorre positivo (resp. negado) em $c_{j}$, então identificamos o vértice $t_{j}^{1}$ de $\mathcal{C}_{j}$, com um vértice $a_{1}^{k}$ (resp. $b_{1}^{k}$ ) de $\mathcal{X}_{1}$ que ainda não foi envolvido em nenhuma identificação (veja a Figura 5.4). Note que $G^{\prime}$ é irredundante e $\Delta\left(G^{\prime}\right)=4$.

Um empacotamento maximal $\mathcal{A}$ de $\left\{K_{2}, K_{3}\right\}$ em $G^{\prime}$ é chamado de canônico se, para cada componente de atribuição, $\mathcal{A}$ contém ou todos os triângulos pares ou todos os triângulos ímpares, e para cada componente de teste $\mathcal{C}_{j}, \mathcal{A}$ contém o triângulo $\left[r_{j}^{1}, r_{j}^{2}, s_{j}^{2}\right]$, e possivelmente uma das arestas $t_{j}^{1} s_{j}^{1}$ ou $t_{j}^{2} s_{j}^{1}$. Inicialmente, provamos a seguinte afirmação.

Dado um empacotamento de $\left\{K_{2}, K_{3}\right\}$ não-canônico $\mathcal{A}$ de $G^{\prime}$, podemos construir em tempo polinomial um empacotamento canônico de $G^{\prime}$ cujo valor é pelo menos o valor de $\mathcal{A}$.

Vamos construir o empacotamento desejado $\mathcal{A}^{\prime}$ a partir de $\mathcal{A}$. Começamos com $\mathcal{A}^{\prime}=\mathcal{A}$. Inicialmente, para cada componente de atribuição $\mathcal{C}_{j}, 1 \leq j \leq l$, removemos de $\mathcal{A}^{\prime}$ os triângulos e arestas que estão em $\mathcal{C}_{j}$ e adicionamos $\left[r_{j}^{1}, r_{j}^{2}, s_{j}^{2}\right]$ a ele. Além disso, se uma das arestas $t_{j}^{1} s_{j}^{1}$, $t_{j}^{2} s_{j}^{1}$ é coberta por $\mathcal{A}$, então adicionamos a $\mathcal{A}^{\prime}$ a aresta coberta por $\mathcal{A}$. Observe que para cada 


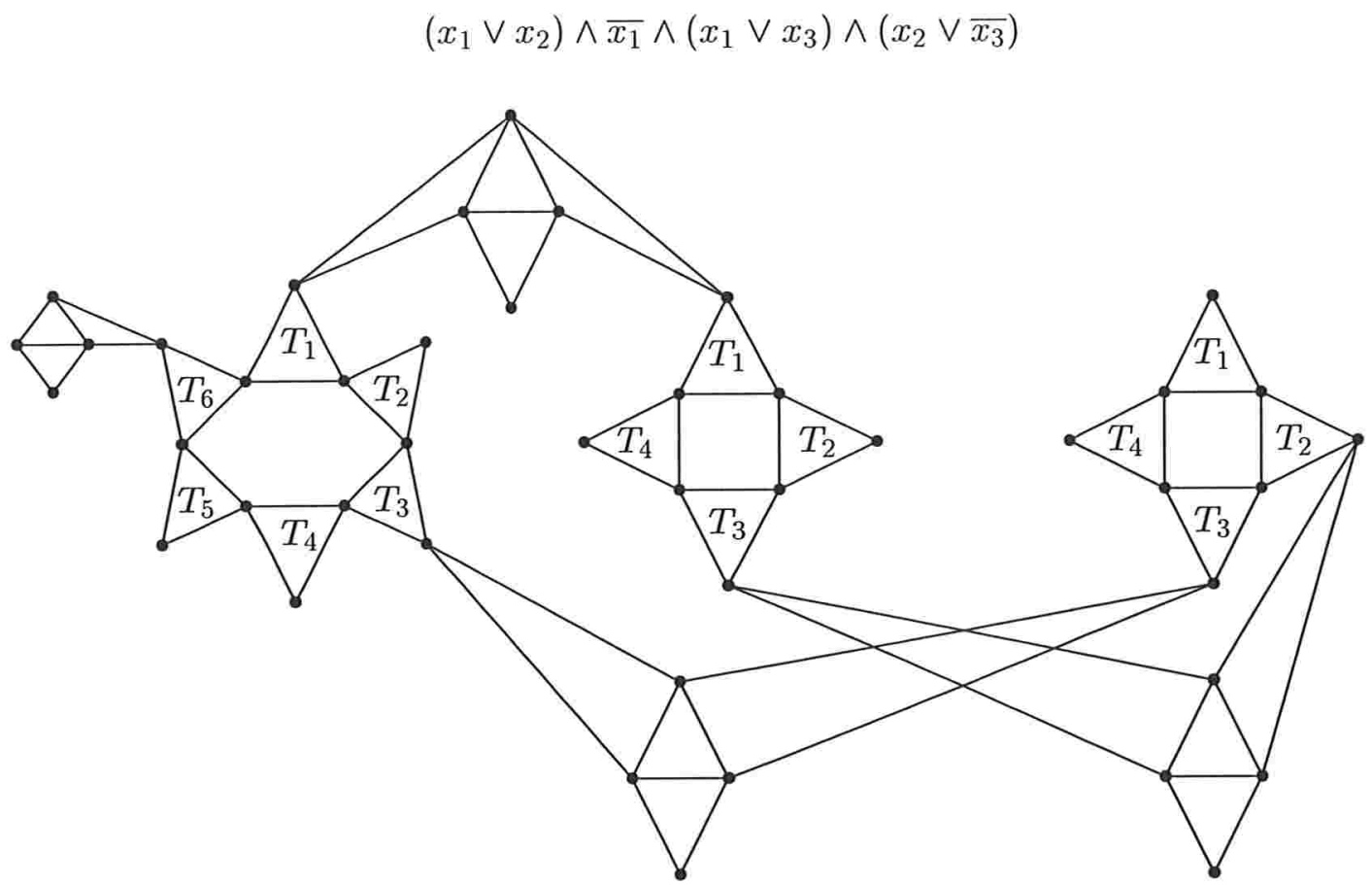

Figura 5.4: Um exemplo de construção de $G^{\prime}$ a partir de uma instância de MAX2SAT3.

$\mathcal{C}_{j}$, o valor de $\mathcal{A}$ restrito a $\mathcal{C}_{j}$ é no máximo 4 . Ainda, se o valor de $\mathcal{A}$ restrito a $\mathcal{C}_{j}$ é exatamente 4 , então uma das arestas $t_{j}^{1} s_{j}^{1}, t_{j}^{2} s_{j}^{1}$ é coberta por $\mathcal{A}$. Portanto, o empacotamento $\mathcal{A}^{\prime}$ obtido até o momento tem um valor que é pelo menos o valor de $\mathcal{A}$.

Além disso, para cada $i, 1 \leq i \leq p$, se os triângulos da componente de atribuição $\mathcal{X}_{i}$ que estão em $\mathcal{T}_{\mathcal{A}}$ não são todos de mesma paridade, fazemos o seguinte (dependendo do número de ocorrências de $x_{i}$ ).

1. $m_{i}=3$.

Podemos supor, sem perda de generalidade, que $x_{i}$ aparece negado em uma cláusula, digamos $c_{j}$, e positivo em duas cláusulas (pois se $x_{i}$ aparece somente negado ou somente positivo, podemos atribuir a $x_{i}$ o valor que satisfaz todas as cláusulas nas quais ela aparece). Seja $t_{j}^{k}, k \in\{1,2\}$, o vértice de $\mathcal{C}_{j}$ que incide em $\mathcal{X}_{i}$. Então, removemos de $\mathcal{A}^{\prime}$ os triângulos e arestas que estão em $\mathcal{X}_{i}$ e adicionamos todos os triângulos pares de $\mathcal{X}_{i}$ em $\mathcal{A}^{\prime}$. Ainda, se $t_{j}^{k} s_{j}^{1}$ está em $\mathcal{A}^{\prime}$, então removemos esta aresta. Mostramos em seguida que após estas mudanças o valor de $\mathcal{A}^{\prime}$ é pelo menos o valor de $\mathcal{A}$.

(a) Se $\mathcal{X}_{i}$ não tem nenhum triângulo que está em $\mathcal{T}_{\mathcal{A}}$, então existem no máximo 6 arestas de $\mathcal{X}_{i}$ que estão em $\mathcal{E}_{A}$, uma de cada triângulo. Portanto, o valor do empacotamento diminui de no máximo 7 . Como o valor do empacotamento aumenta de 9 , temos que o valor de $\mathcal{A}^{\prime}$ aumenta. 
(b) Se existe exatamente um triângulo de $\mathcal{X}_{i}$ que está em $\mathcal{T}_{\mathcal{A}}$, então, existem no máximo 5 arestas de $\mathcal{X}_{i}$ que estão em $\mathcal{E}_{A}$, uma de cada triângulo. Logo, o valor de $\mathcal{A}^{\prime}$ diminui de no máximo 9 , e aumenta de 9 .

(c) Se existem exatamente dois triângulos de $\mathcal{X}_{i}$ que estão em $\mathcal{T}_{\mathcal{A}}$, então, existem no máximo duas arestas de $\mathcal{X}_{i}$ que estão em $\mathcal{E}_{A}$ (veja exemplos nas Figuras 5.5(a) e (b)). Portanto, temos que o valor do empacotamento $\mathcal{A}^{\prime}$ diminui de no máximo 9 , e aumenta de 9 .

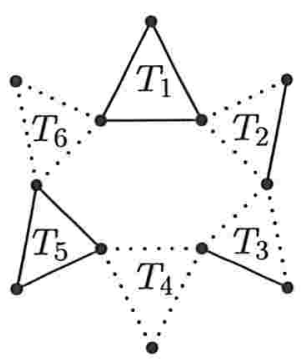

(a)

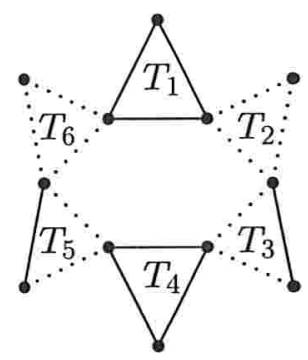

(b)

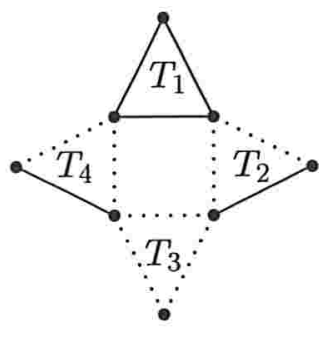

(c)

Figura 5.5: Triângulos e arestas com linhas cheias estão em $\mathcal{A}$. (a) $m_{i}=3$ existem exatamente dois triângulos de $\mathcal{X}_{i}$ de mesma paridade que estão em $\mathcal{T}_{\mathcal{A}}$. (b) $m_{i}=3$ e existem exatamente dois triângulos de $\mathcal{X}_{i}$ de paridades diferentes que estão em $\mathcal{T}_{\mathcal{A}}$. (c) $m_{i}=2$ e existe um triângulo de $\mathcal{X}_{i}$ que está em $\mathcal{T}_{\mathcal{A}}$. Em todos os casos, existem no máximo 2 arestas de $\mathcal{X}_{i}$ que estão em $\mathcal{E}_{A}$.

2. $m_{i}=2$.

Podemos supor, sem perda de generalidade, que $x_{i}$ aparece negado em uma cláusula, digamos $c_{j}$, e positivo em outra. Então, removemos de $\mathcal{A}^{\prime}$ os triângulos e as arestas que estão em $\mathcal{X}_{i}$ e adicionamos dois triângulos pares de $\mathcal{X}_{i}$ em $\mathcal{A}^{\prime}$. Além disso, se $t_{j}^{1} s_{j}^{1}$ está em $\mathcal{A}^{\prime}$, removemos esta aresta. Mostramos em seguida que estas mudanças resultam em um empacotamento $\mathcal{A}^{\prime}$ cujo valor é pelo menos o valor de $\mathcal{A}$.

(a) Se nenhum triângulo de $\mathcal{X}_{i}$ está em $\mathcal{T}_{\mathcal{A}}$, então existem no máximo 4 arestas de $\mathcal{X}_{i}$ que estão em $\mathcal{E}_{A}$, uma de cada triângulo. Portanto, o valor do empacotamento $\mathcal{A}^{\prime}$ diminui de no máximo 5 . Como o valor do empacotamento aumenta de 6 , temos que o valor de $\mathcal{A}^{\prime}$ aumenta.

(b) Se existe um triângulo de $\mathcal{X}_{i}$ que está em $\mathcal{T}_{\mathcal{A}}$, então existe somente um tal triângulo, digamos $T_{k}$. Além disso, existem no máximo 2 arestas de $\mathcal{X}_{i}$ que estão em $\mathcal{E}_{A}$, pois o número de vértices em $\mathcal{X}_{i}-V_{T_{k}}$ é 5 (veja um exemplo na Figura 5.5(c)). Portanto, o valor de $\mathcal{A}^{\prime}$ diminui de no máximo 6 , e aumenta de 6 .

Finalmente, para cada componente de teste $\mathcal{C}_{j}$, se $s_{j}^{1}$ não é ainda o extremo de uma aresta em $\mathcal{E}_{\mathcal{A}^{\prime}}$, então sempre que possível, adicionamos uma das arestas $t_{j}^{1} s_{j}^{1}$ ou $t_{j}^{2} s_{j}^{1}$ em $\mathcal{A}^{\prime}$. Ou seja, se a cláusula correspondente $c_{j}$ tem dois literais, então, se $t_{j}^{1}$ não está coberto por $\mathcal{A}^{\prime}$, adicionamos $t_{j}^{1} s_{j}^{1}$ em $\mathcal{A}^{\prime}$; caso contrário, se $t_{j}^{2}$ não está coberto por $\mathcal{A}^{\prime}$, adicionamos $t_{j}^{2} s_{j}^{1}$ a $\mathcal{A}^{\prime}$. Se, no entanto, a cláusula $c_{j}$ tem somente um literal, então se $t_{j}^{1}$ não está coberto por $\mathcal{A}^{\prime}$, adicionamos $t_{j}^{1} s_{j}^{1}$ ao $\mathcal{A}^{\prime}$.

Na Figura 5.6 exibimos um empacotamento $\mathcal{A}$ e o empacotamento $\mathcal{A}^{\prime}$ obtido a partir de $\mathcal{A}$. 
Note que o empacotamento obtido $\mathcal{A}^{\prime}$ é um empacotamento canônico de $G^{\prime}$ cujo valor é pelo menos o valor de $\mathcal{A}$. Provamos portanto a afirmação (5.4).

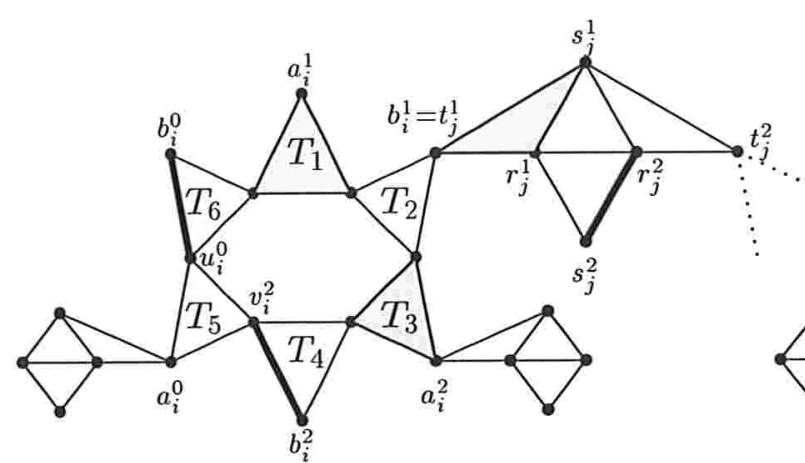

(a)

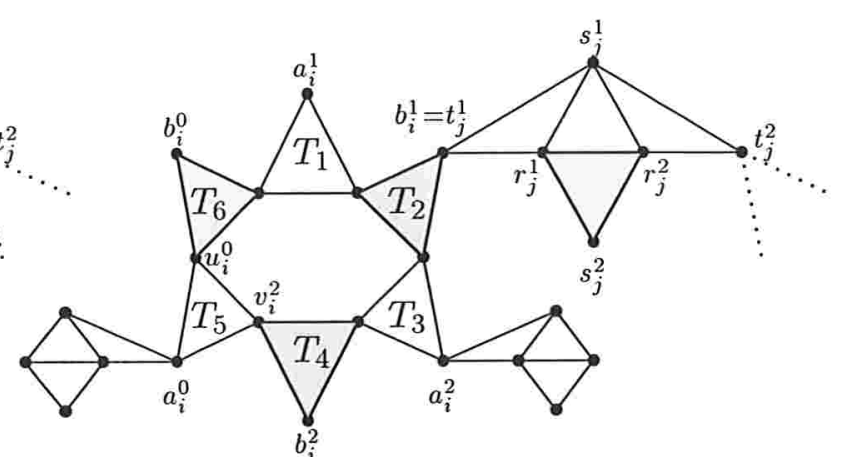

(b)

Figura 5.6: Um exemplo de construção de $\mathcal{A}^{\prime}$ (quando $m_{i}=3$ e $x_{i}$ aparece negado em somente uma cláusula, digamos $c_{j}$, e positivo em duas outras cláusulas). Linhas pontilhadas indicam arestas de uma outra componente de atribuição. (a) Mostra um empacotamento $\mathcal{A}$ de $\left\{K_{2}, K_{3}\right\}$ não-canônico restrito ao $\mathcal{X}_{i}$ e $\mathcal{C}_{j}$ (arestas e triângulos destacados estão em $\mathcal{A}$ ). Na primeira fase, $\left[t_{j}^{1}, s_{j}^{1}, r_{j}^{1}\right]$ e $s_{j}^{2} r_{j}^{2}$ são removidos de $\mathcal{A}^{\prime}$, e $\left[r_{j}^{1}, r_{j}^{2}, s_{j}^{2}\right]$ e $t_{j}^{1} s_{j}^{1}$ são adicionados a $\mathcal{A}^{\prime}$. No segundo passo, $T_{1}, T_{3}, u_{i}^{0} b_{i}^{0}, v_{i}^{2} b_{i}^{2}, t_{j}^{1} s_{j}^{1}$ são removidos de $\mathcal{A}^{\prime}$ e os triângulos $T_{2}, T_{4}, T_{6}$ são adicionados a $\mathcal{A}^{\prime}$. (b) $\mathrm{O}$ empacotamento $\mathcal{A}^{\prime}$ obtido.

Observamos que um dado empacotamento canônico $\mathcal{A}^{\prime}$ de $G^{\prime}$ corresponde a uma atribuição (verdadeiro/falso) para as variáveis em $X$. De fato, se $\mathcal{A}^{\prime}$ contém todos os triângulos pares (resp. ímpares) de uma componente de atribuição $\mathcal{X}_{i}$, então $x_{i}$ consideramos verdadeiro (resp. falso). Por outro lado, dada uma atribuição para $X$, podemos construir um empacotamento canônico $\mathcal{A}^{\prime}$ de $G^{\prime}$ da seguinte maneira. Se $x_{i}$ é verdadeiro (resp. falso), adicionamos todos os triângulos pares (resp. ímpares) de $\mathcal{X}_{i}$ a $\mathcal{A}^{\prime}$. Para cada componente de teste $\mathcal{C}_{j}$, adicionamos o triângulo $\left[r_{j}^{1}, r_{j}^{2}, s_{j}^{2}\right]$ a $\mathcal{A}^{\prime}$. Além disso, se a cláusula correspondente $c_{j}$ tem dois literais, então se $t_{j}^{1}$ não está coberto por $\mathcal{A}^{\prime}$ adicionamos $t_{j}^{1} s_{j}^{1}$ a $\mathcal{A}^{\prime}$; caso contrário, se $t_{j}^{2}$ não está coberto por $\mathcal{A}^{\prime}$ adicionamos $t_{j}^{2} s_{j}^{1}$ ao empacotamento. Se, no entanto, a cláusula $c_{j}$ tem somente um literal, então se $t_{j}^{1}$ não está coberto por $\mathcal{A}^{\prime}$ adicionamos $t_{j}^{1} s_{j}^{1}$ a $\mathcal{A}^{\prime}$ (veja a Figura 5.7).

Considere agora um empacotamento canônico $\mathcal{A}^{\prime}$ e a correspondente atribuição (verdadeiro/falso) para as variáveis de $X$. Seja $c_{j}$ uma cláusula com dois literais, e sejam $x_{1}, x_{2}$ as variáveis que ocorrem em $c_{j}$. Note que $t_{j}^{i}$ (para $\left.i=1,2\right)$ não está coberto por nenhum triângulo em $\mathcal{A}^{\prime}$ que pertence ao componente da atribuição correspondente $\mathcal{X}_{i}$, se e somente se, $x_{i}$ satisfaz $c_{j}$. Portanto, da construção do empacotamento canônico temos que as seguintes afirmações são equivalentes:

- a cláusula $c_{j}$ é satisfatível,

- pelo menos um dos vértices $t_{j}^{1}, t_{j}^{2}$ não está coberto por um triângulo em $\mathcal{A}^{\prime}$ que pertence ao componente da atribuição correspondente,

- exatamente uma das arestas $t_{j}^{1} s_{j}^{1}, t_{j}^{2} s_{j}^{1}$ está em $\mathcal{E}_{\mathcal{A}^{\prime}}$,

- o valor de $\mathcal{A}^{\prime}$ restrito a $\mathcal{C}_{j}$ é 4 . 


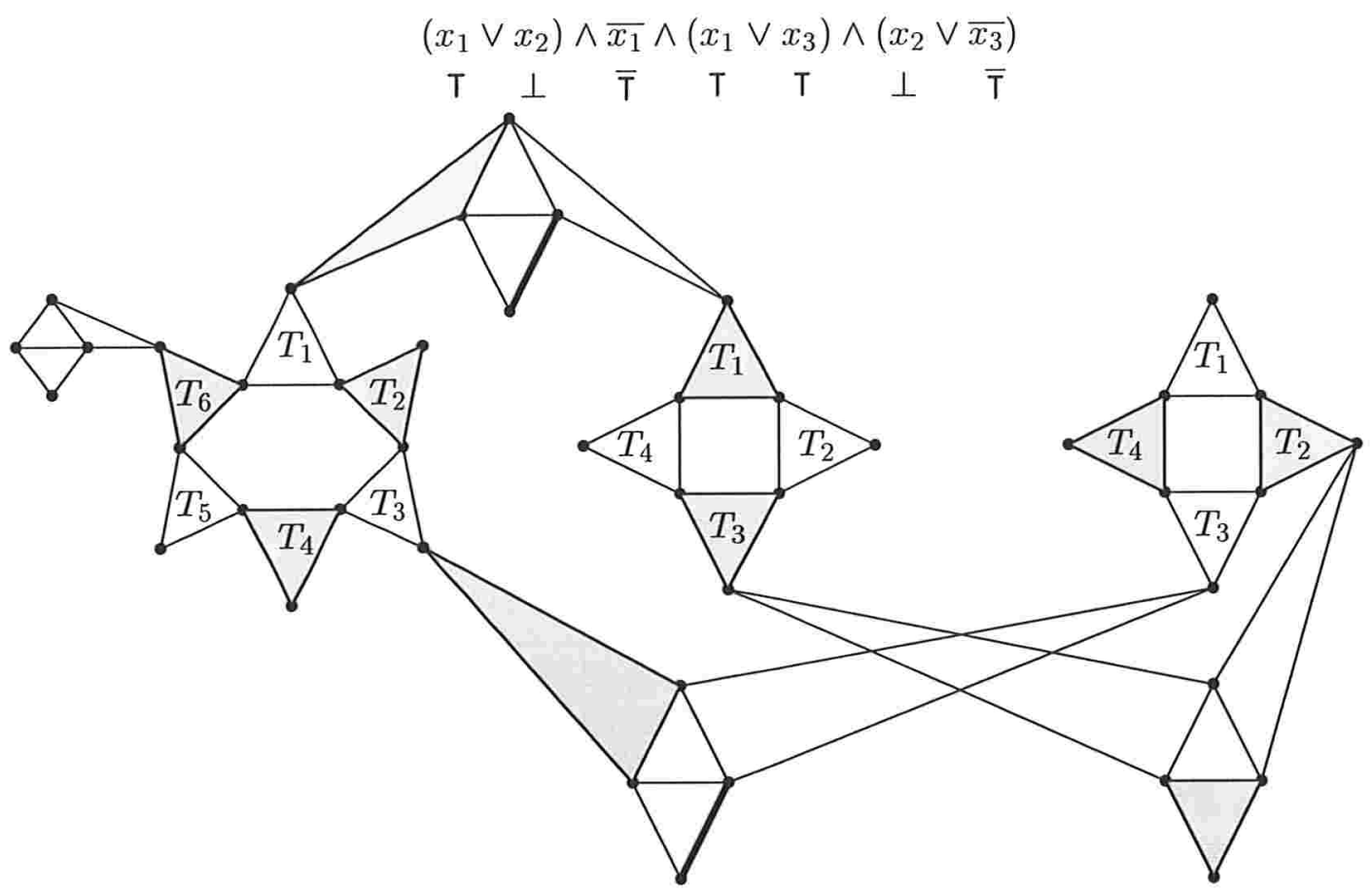

Figura 5.7: Um exemplo de uma atribuição para $X$ e a correspondente empacotamento canônico $\mathcal{A}^{\prime}$ de $G^{\prime}$.

Afirmações similares são verdadeiras para cláusulas com somente um literal. Portanto, o valor de $\mathcal{A}^{\prime}$ restrito a $\mathcal{C}_{j}$ é 4 (resp. 3), se e somente se, $c_{j}$ é satisfatível (resp. não-satisfatível). Além disso, exatamente $m_{i}$ triângulos de cada $\mathcal{X}_{i}$ estão em $\mathcal{A}^{\prime}$. Logo, temos que a seguinte afirmação é válida.

Um empacotamento canônico $\mathcal{A}^{\prime}$ de $G^{\prime}$ com valor $\sum_{i=1}^{p} 3 m_{i}+4 k+3(l-k)$ corresponde a uma atribuição para as variáveis de $X$ que satisfaz exatamente $k$ cláusulas de $\varphi$, e vice-versa.

Agora, dado um empacotamento $\mathcal{A}$ de $\left\{K_{2}, K_{3}\right\}$ em $G^{\prime}:=f(\varphi)$, definimos uma atribuição $g(\varphi, \mathcal{A})$ do seguinte modo. Inicialmente, encontramos um empacotamento canônico $\mathcal{A}^{\prime}$ de $G^{\prime}$ cujo valor é pelo menos o valor de $\mathcal{A}$. Atribuímos a $x_{i}$ o valor verdadeiro (resp. falso) se $\mathcal{A}^{\prime}$ contém todos os triângulos pares (resp. ímpares) da componente de atribuição $\mathcal{X}_{i}$.

Mostramos em seguida que

$$
\operatorname{opt}_{\mathrm{K}_{2}+\mathrm{K}_{3}}\left(G^{\prime}\right)=\sum_{i=1}^{p} 3 m_{i}+\operatorname{opt}_{\mathrm{MAX} 2 \mathrm{SAT} 3}(\varphi)+3 l .
$$

De fato, de (5.5) temos que uma solução ótima de $\operatorname{MAX2SAT3}(\varphi)$ corresponde a um empacotamento canônico $\mathcal{A}^{\prime}$ de $G^{\prime}$ com valor $\sum_{i=1}^{p} 3 m_{i}+4 \operatorname{opt}_{\mathrm{MAX2SAT} 3}(\varphi)+3\left(l-\operatorname{opt}_{\mathrm{MAX} 2 \mathrm{SAT} 3}(\varphi)\right)$. 
Portanto, $\operatorname{opt}_{\mathrm{K}_{2}+\mathrm{K}_{3}}\left(G^{\prime}\right) \geq \sum_{i=1}^{p} 3 m_{i}+\operatorname{opt}_{\mathrm{MAX} 2 \mathrm{SAT} 3}(\varphi)+3 l$. Por outro lado, seja $\mathcal{A}$ um $\left\{K_{2}, K_{3}\right\}$-empacotamento de $G^{\prime}$. Se a solução viável correspondente $g(\varphi, \mathcal{A})$ de $\operatorname{MAX} 2 \operatorname{SAT} 3(\varphi)$ satisfaz $k$ cláusulas, temos que $k \leq \operatorname{opt}_{\mathrm{MAX} 2 \mathrm{SAT} 3}(\varphi)$. Ainda, $\operatorname{val}_{\mathrm{K}_{2}+\mathrm{K}_{3}}\left(G^{\prime}, \mathcal{A}\right) \leq \operatorname{val}_{\mathrm{K}_{2}+\mathrm{K}_{3}}\left(G^{\prime}, \mathcal{A}^{\prime}\right)$, e por $(5.5), \operatorname{val}_{K_{2}+K_{3}}\left(G^{\prime}, \mathcal{A}^{\prime}\right)=\sum_{i=1}^{p} 3 m_{i}+k+3 l$. Portanto, temos que $\operatorname{opt}_{\mathrm{K}_{2}+\mathrm{K}_{3}}\left(G^{\prime}\right) \leq$ $\sum_{i=1}^{p} 3 m_{i}+\operatorname{opt}_{\operatorname{MAX} 2 \mathrm{SAT} 3}(\varphi)+3 l$. Provamos então a afirmação (5.6).

Como cada cláusula tem no máximo 2 literais, temos que $\sum_{i=1}^{p} m_{i} \leq 2 l$. Além disso, note que o valor ótimo para instância $\varphi$ do problema MAX2SAT3 é pelo menos $\frac{l}{2}$, pois pelo menos metade das cláusulas pode ser satisfeita usando-se um algoritmo guloso simples. Logo, de (5.6) temos que $\operatorname{opt}_{\mathrm{K}_{2}+\mathrm{K}_{3}}\left(G^{\prime}\right) \leq 9 l+\operatorname{opt}_{\mathrm{MAX} 2 \mathrm{SAT} 3}(\varphi) \leq 19 \operatorname{opt}_{\mathrm{MAX} 2 \mathrm{SAT} 3}(\varphi)$, ou seja, $\alpha=19$.

Finalmente, suponha que val $\operatorname{MAX2SAT}_{3}(\varphi, g(\varphi, \mathcal{A}))=k$, isto é, que a atribuição $g(\varphi, \mathcal{A})$ satisfaz exatamente $k$ cláusulas de $\varphi$. Portanto, por (5.5) temos val $\operatorname{K}_{2}+\mathrm{K}_{3}\left(G^{\prime}, \mathcal{A}^{\prime}\right)=\sum_{i=1}^{p} 3 m_{i}+$ $k+3 l$. Desta afirmação, da igualdade (5.6), e do fato de que $\operatorname{val}_{\mathrm{K}_{2}+\mathrm{K}_{3}}\left(G^{\prime}, \mathcal{A}^{\prime}\right) \geq \operatorname{val}_{\mathrm{K}_{2}+\mathrm{K}_{3}}\left(G^{\prime}, \mathcal{A}\right)$, temos que

$$
\operatorname{opt}_{\mathrm{MAX} 2 \mathrm{SAT} 3}(\varphi)-\operatorname{val}_{\mathrm{MAX} 2 \mathrm{SAT} 3}(\varphi, g(\varphi, \mathcal{A})) \leq \operatorname{opt}_{\mathrm{K}_{2}+\mathrm{K}_{3}}\left(G^{\prime}\right)-\operatorname{val}_{\mathrm{K}_{2}+\mathrm{K}_{3}}\left(G^{\prime}, \mathcal{A}\right) .
$$

Portanto, $\beta=1$. O resultado do teorema segue do fato de que o problema $\mathrm{K}_{2}+\mathrm{K}_{3^{-}}$ empacotamento pertence a classe APX e MAX2SAT3 é APX-completo [4].

\subsection{Algoritmo de aproximação}

Apresentamos nesta seção um algoritmo que desenvolvemos para o problema do $\mathrm{K}_{2}+\mathrm{K}_{3}$ empacotamento, denominado $\mathrm{K}_{2} \mathrm{~K}_{3}$. Dado um grafo $G$, o algoritmo $\mathrm{K}_{2} \mathrm{~K}_{3}$ inicialmente encontra em $G$ uma coleção de triângulos disjuntos nos vértices, aplicando um algoritmo de aproximação $A_{\mathrm{VTP}}$ para o problema VTP. Em seguida, o algoritmo $\mathrm{K}_{2} \mathrm{~K}_{3}$ complementa a solução com um emparelhamento máximo no grafo obtido após remover os triângulos que foram encontrados pelo algoritmo $A_{\mathrm{VTP}}$.

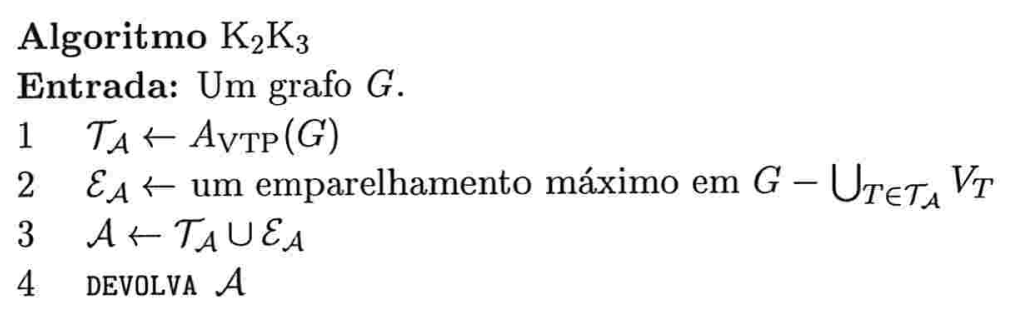

Teorema 5.3 Seja $A_{\mathrm{VTP}}$ uma $\rho$-aproximação para o problema VTP que devolve, para cada grafo de entrada $G$, uma coleção maximal de triângulos disjuntos nos vértices em $G$. Então, o algoritmo $\mathrm{K}_{2} \mathrm{~K}_{3}$ é uma $\left(1+\frac{1}{3} \rho\right)$-aproximação para o problema do $\mathrm{K}_{2}+\mathrm{K}_{3}$-empacotamento.

Demonstração. Seja $G$ um grafo e $\mathcal{A}$ a solução devolvida pelo algoritmo $\mathrm{K}_{2} \mathrm{~K}_{3}$ quando aplicado a $G$. Seja $\mathcal{O}$ uma solução ótima para o $\mathrm{K}_{2}+\mathrm{K}_{3}$-empacotamento de $G$ com o maior número possível de triângulos em comum com $\mathcal{A}$. Usamos os seguintes números:

- $t$ : o número de triângulos comuns a $\mathcal{A}$ e $\mathcal{O}$. 
- $t_{3}$ : o número de triângulos de $\mathcal{A}$ que incidem em 3 triângulos diferentes de $\mathcal{O}$.

- $t_{2}^{v}$ : o número de triângulos de $\mathcal{A}$ que incidem em exatamente 2 triângulos diferentes de $\mathcal{O}$, tendo precisamente um vértice em comum com ambos.

- $t_{2}^{e}$ : o número de triângulos de $\mathcal{A}$ que incidem em exatamente 2 triângulos diferentes de $\mathcal{O}$, tendo uma aresta em comum com um deles.

- $t_{1}^{v}$ : o número de triângulos de $\mathcal{A}$ que incidem em exatamente 1 triângulo de $\mathcal{O}$, tendo precisamente um vértice em comum com ele.

- $t_{1}^{e}$ : o número de triângulos de $\mathcal{A}$ que incidem em exatamente 1 triângulo de $\mathcal{O}$, tendo precisamente uma aresta em comum com ele.

- $t_{0}$ : o número de triângulos de $\mathcal{A}$ que não incidem em nenhum triângulo de $\mathcal{O}$.

Mostramos inicialmente que $t_{0}=0$. Suponha que $t_{0}>0$ e que $T$ é um triângulo de $\mathcal{A}$ que não incide em nenhum triângulo de $\mathcal{O}$. Se no máximo duas arestas de $\mathcal{E}_{\mathcal{O}}$ são adjacentes a $T$, então substituindo estas arestas por $T$, obtemos um $\left\{K_{2}, K_{3}\right\}$-empacotamento com valor maior que $\mathcal{O}$, uma contradição. Portanto, existem 3 arestas de $\mathcal{E}_{\mathcal{O}}$ adjacentes a $T$. Removendo estas arestas e adicionando $T$ em $\mathcal{O}$, obtemos uma solução ótima do $\mathrm{K}_{2}+\mathrm{K}_{3}$-empacotamento que tem mais triângulos em comum $\operatorname{com} \mathcal{A}$ do que $\mathcal{O}$, o que é novamente uma contradição. Logo, $t_{0}=0$.

Introduzimos agora números similares para os triângulos de $\mathcal{O}$.

- o: o número de triângulos comuns a $\mathcal{O}$ e $\mathcal{A}$.

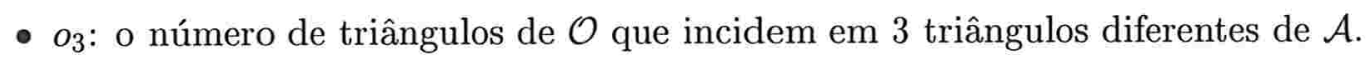

- $o_{2}^{v}$ : o número de triângulos de $\mathcal{O}$ que incidem em exatamente 2 triângulos diferentes de $\mathcal{A}$, tendo precisamente um vértice em comum com ambos.

- $o_{2}^{e}$ : o número de triângulos de $\mathcal{O}$ que incidem em exatamente 2 triângulos diferentes de $\mathcal{A}$, tendo uma aresta em comum com um deles.

- olv o número de triângulos de $\mathcal{O}$ que incidem em exatamente 1 triângulo de $\mathcal{A}$, tendo precisamente um vértice em comum com ele.

- o $o_{1}^{e}$ o número de triângulos de $\mathcal{O}$ que incidem em exatamente 1 triângulo de $\mathcal{A}$, tendo precisamente uma aresta em comum com ele.

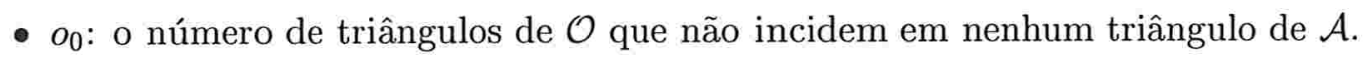

Como o algoritmo $A_{\mathrm{VTP}}$ devolve um empacotamento de triângulos maximal, $o_{0}$ é zero. Mostramos em seguida que

$$
3 t+3 t_{3}+2 t_{2}^{v}+2 t_{2}^{e}+t_{1}^{v}+t_{1}^{e}=3 o+3 o_{3}+2 o_{2}^{v}+2 o_{2}^{e}+o_{1}^{v}+o_{1}^{e} .
$$

De fato, note que um triângulo de $\mathcal{A}$ e um triângulo de $\mathcal{O}$ podem ter exatamente um dos seguintes em comum: precisamente um vértice, precisamente uma aresta, ou três vértices (no caso em que um triângulo está em $\mathcal{A}$ e $\mathcal{O}$ ). Seja $I$ o número de incidências entre os triângulos de $\mathcal{A}$ e $\mathcal{O}$, ou seja, $I$ é o valor obtido pela contagem do número de arestas e vértices em comum aos triângulos de $\mathcal{A}$ e $\mathcal{O}$ (no caso em que um triângulo de $\mathcal{A}$ e um triângulo de $\mathcal{O}$ 
têm precisamente uma aresta em comum, incrementamos de 1 o valor de $I$, e não contamos os vértices desta aresta). Claramente, o valor de cada lado da equação (5.7) é igual a $I$.

Agora, contando as arestas que estão na interseção de um triângulo em $\mathcal{A}$ e um triângulo em $\mathcal{O}$, obtemos $3 t+t_{2}^{e}+t_{1}^{e}=3 o+o_{2}^{e}+o_{1}^{e}$. Como $t=o$, temos

$$
t_{2}^{e}+t_{1}^{e}=o_{2}^{e}+o_{1}^{e} .
$$

Introduzimos agora os números que usaremos para as arestas em $\mathcal{E}_{\mathcal{O}}$.

- $e_{\mathcal{O}}^{\mathcal{T}}$ : o número de arestas em $\mathcal{E}_{\mathcal{O}}$ que têm pelo menos um vértice em comum com um triângulo de $\mathcal{A}$.

- $e_{\mathcal{O}}$ : o número de arestas em $\mathcal{E}_{\mathcal{O}}$ que não têm nenhum vértice em comum com algum triângulo de $\mathcal{A}$.

Note que $e_{\mathcal{O}}^{\mathcal{T}}$ é no máximo o número de vértices dos triângulos de $\mathcal{A}$ não-cobertos por nenhum triângulo de $\mathcal{O}$, isto é,

$$
e_{\mathcal{O}}^{\mathcal{T}} \leq 2 t_{1}^{v}+t_{2}^{v}+t_{1}^{e}
$$

Seja $G^{\prime}$ o grafo obtido removendo-se todos os vértices dos triângulos de $\mathcal{A}$, ou seja, $G^{\prime}:=$ $G-\bigcup_{T \in \mathcal{T}_{\mathcal{A}}} V_{T}$. Note que um emparelhamento de $G^{\prime}$ pode ser obtido tomando-se uma aresta de cada triângulo de $\mathcal{O}$ que tenha exatamente um vértice em comum com um triângulo de $\mathcal{A}$, e tomando-se as arestas de $\mathcal{E}_{\mathcal{O}}$ que não têm nenhum vértice em comum com um triângulo de $\mathcal{A}$. Portanto, como $\mathcal{E}_{\mathcal{A}}$ é um empacotamento máximo de $G^{\prime}$, temos que $\left|\mathcal{E}_{\mathcal{A}}\right| \geq o_{1}^{v}+e_{\mathcal{O}}$. Deste fato e da desigualdade (5.9), temos que

$$
\left|\mathcal{E}_{\mathcal{O}}\right|=e_{\mathcal{O}}^{\mathcal{T}}+e_{\mathcal{O}} \leq 2 t_{1}^{v}+t_{2}^{v}+t_{1}^{e}+\left|\mathcal{E}_{\mathcal{A}}\right|-o_{1}^{v}
$$

Consideramos agora a razão do valor de $\mathcal{O}$ sobre o valor de $\mathcal{A}$,

$$
r=\frac{3\left|\mathcal{T}_{\mathcal{O}}\right|+\left|\mathcal{E}_{\mathcal{O}}\right|}{3\left|\mathcal{T}_{\mathcal{A}}\right|+\left|\mathcal{E}_{\mathcal{A}}\right|}
$$

Substituindo $\left|\mathcal{T}_{\mathcal{O}}\right|$ e usando (5.10), obtemos

$$
r \leq \frac{3\left(o+o_{3}+o_{2}^{v}+o_{2}^{e}+o_{1}^{v}+o_{1}^{e}\right)+\left(2 t_{1}^{v}+t_{2}^{v}+t_{1}^{e}+\left|\mathcal{E}_{\mathcal{A}}\right|-o_{1}^{v}\right)}{3\left|\mathcal{T}_{\mathcal{A}}\right|+\left|\mathcal{E}_{\mathcal{A}}\right|} .
$$

Como $\left|\mathcal{E}_{\mathcal{A}}\right| \geq 0$ e $r \geq 1$, podemos remover $\left|\mathcal{E}_{\mathcal{A}}\right|$ na última desigualdade, obtendo

$$
r \leq \frac{3\left(o+o_{3}+o_{2}^{v}+o_{2}^{e}+o_{1}^{v}+o_{1}^{e}\right)+\left(2 t_{1}^{v}+t_{2}^{v}+t_{1}^{e}-o_{1}^{v}\right)}{3\left|\mathcal{T}_{\mathcal{A}}\right|} .
$$

Usando (5.7), temos

$$
\begin{gathered}
r \leq \frac{\left(3 t+3 t_{3}+2 t_{2}^{v}+2 t_{2}^{e}+t_{1}^{v}+t_{1}^{e}\right)+\left(o_{2}^{v}+o_{2}^{e}+2 o_{1}^{v}+2 o_{1}^{e}\right)+\left(2 t_{1}^{v}+t_{2}^{v}+t_{1}^{e}-o_{1}^{v}\right)}{3\left|\mathcal{T}_{\mathcal{A}}\right|} \\
=\frac{3 t+3 t_{3}+3 t_{2}^{v}+2 t_{2}^{e}+3 t_{1}^{v}+2 t_{1}^{e}+o_{2}^{v}+o_{2}^{e}+o_{1}^{v}+2 o_{1}^{e}}{3\left|\mathcal{T}_{\mathcal{A}}\right|}
\end{gathered}
$$


Agora, de (5.8) temos

$$
r \leq \frac{3\left(t+t_{3}+t_{2}^{v}+t_{2}^{e}+t_{1}^{v}+t_{1}^{e}\right)+\left(o_{2}^{v}+o_{1}^{v}+o_{1}^{e}\right)}{3\left|\mathcal{T}_{\mathcal{A}}\right|}=\frac{3\left|\mathcal{T}_{\mathcal{A}}\right|+\left(o_{2}^{v}+o_{1}^{v}+o_{1}^{e}\right)}{3\left|\mathcal{T}_{\mathcal{A}}\right|} .
$$

Como $o_{2}^{v}+o_{1}^{v}+o_{1}^{e} \leq o+o_{3}+o_{2}^{v}+o_{2}^{e}+o_{1}^{v}+o_{1}^{e}=\left|\mathcal{T}_{\mathcal{O}}\right|$, temos $r \leq 1+\frac{1}{3} \frac{\left|\mathcal{T}_{\mathcal{O}}\right|}{\left|\mathcal{T}_{\mathcal{A}}\right|}$. Como $\left|\mathcal{T}_{\mathcal{O}}\right| \leq \operatorname{opt}_{\mathrm{VTP}}(G)$ e $A_{\mathrm{VTP}}$ é uma $\rho$-aproximação para o problema VTP,

$$
\frac{\left|\mathcal{T}_{\mathcal{O}}\right|}{\left|\mathcal{T}_{\mathcal{A}}\right|} \leq \frac{\operatorname{opt}_{\mathrm{VTP}}(G)}{\left|\mathcal{T}_{\mathcal{A}}\right|} \leq \rho, \text { e portanto } r \leq 1+\frac{1}{3} \rho
$$

Corolário 5.4 Existe uma $\left(\frac{3}{2}+\varepsilon\right)$-aproximação para o problema do $\mathrm{K}_{2}+\mathrm{K}_{3}$-empacotamento.

Demonstração. Aplicando o algoritmo $\mathrm{K}_{2} \mathrm{~K}_{3}$ com $A_{\mathrm{VTP}}=\mathrm{HS}(t)$, o resultado segue do Teorema 5.3 e do fato de que o algoritmo de Hurkens e Schrijver, HS(t), é um algoritmo de $\left(\frac{3}{2}+\varepsilon\right)$-aproximação para o problema VTP [23].

Corolário 5.5 Existe um algoritmo de 1,4-aproximação para o problema do $\mathrm{K}_{2}+\mathrm{K}_{3}$-empacotamento em grafos de grau máximo 4.

Demonstração. Segue do Teorema 5.3 e do Teorema 3.15.

\subsection{Observações finais}

$\mathrm{O}$ algoritmo $\mathrm{K}_{2} \mathrm{~K}_{3}$ tem razão de aproximação pelo menos $3 / 2$ quando $A_{\mathrm{VTP}}(G)=\operatorname{HS}(\mathcal{T}, t)$. Mais precisamente, para o grafo $G$ mencionado na seção 3.3 .5 (com 152 triângulos), que é uma instância justa para o algoritmo $\operatorname{HS}\left(\mathcal{T}_{V}(G), 4\right)$, temos que o valor de $\operatorname{opt}_{\mathrm{K}_{2}+\mathrm{K}_{3}}(G)$ sobre o valor da solução obtida pelo algoritmo $\mathrm{K}_{2} \mathrm{~K}_{3}$ é $3 / 2$.

Notamos ainda que uma solução ótima para a versão do $\left\{K_{2}, K_{3}\right\}$-empacotamento na qual o objetivo é encontrar um subgrafo $H$ de $G$ que seja uma união de grafos disjuntos nos vértices e tal que $H$ tenha o maior número possível de vértices não é necessariamente uma boa solução para o nosso problema. Por exemplo, existem instâncias para o nosso problema para as quais a razão entre o valor de uma solução ótima e o número de arestas em um emparelhamento perfeito (solução ótima dessa nova versão) é 2. Veja a Figura 5.8 .

Os resultados que apresentamos nas seções anteriores foram submetidos ao 9th International Workshop on Approximation Algorithms for Combinatorial Optimization Problems (APPROX 2006). Dessa forma, tomamos conhecimento de um resultado de Halldórsson [16] para um problema de cobertura mínima por 3-conjuntos que fornece uma 3/2-aproximação para o problema do $\mathrm{K}_{2}+\mathrm{K}_{3}$-empacotamento. Além disso, Raphael Yuster, um dos pareceristas, sugeriu uma outra 3/2-aproximação para esse problema, cuja análise tem semelhanças com a análise que apresentamos para o nosso algoritmo. 


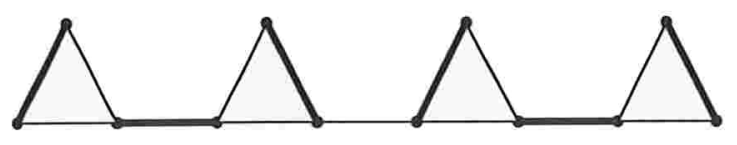

Figura 5.8: As arestas destacadas estão em um emparelhamento perfeito. Os triângulos destacados estão em uma solução ótima do $\mathrm{K}_{2}+\mathrm{K}_{3}$-empacotamento.

Apresentamos em seguida algoritmo sugerido por Raphael Yuster. Inicialmente, o algoritmo encontra em um dado grafo $G$ triângulos disjuntos nos vértices de forma gulosa; em seguida, o algoritmo completa a solução com um emparelhamento máximo no grafo obtido removendo os triângulos que foram encontrados na primeira fase. Para mostrar que esse algoritmo é uma 3/2-aproximação para o problema do $\mathrm{K}_{2}+\mathrm{K}_{3}$-empacotamento, considere uma solução $\mathcal{A}$ devolvida pelo algoritmo de aproximação quando aplicado a um grafo $G$, e uma solução ótima $\mathcal{O}$ para o $\mathrm{K}_{2}+\mathrm{K}_{3}$-empacotamento de $G$. Seja $o_{i}, i=1,2,3$, o número de triângulos de $\mathcal{O}$ que intersectam exatamente $i$ vértices de $\mathcal{T}_{\mathcal{A}}$. Claramente,

$$
o_{1}+o_{2}+o_{3}=\left|\mathcal{T}_{\mathcal{O}}\right| \text {. }
$$

Introduzimos agora os números que usaremos para as arestas em $\mathcal{E}_{\mathcal{O}}$.

- $e_{\mathcal{O}}^{\mathcal{T}}$ : o número de arestas em $\mathcal{E}_{\mathcal{O}}$ que têm pelo menos um vértice em comum com um triângulo de $\mathcal{A}$.

- $e_{\mathcal{O}}$ : o número de arestas em $\mathcal{E}_{\mathcal{O}}$ que não têm nenhum vértice em comum com os triângulos de $\mathcal{A}$.

Observe que $e_{\mathcal{O}}^{\mathcal{T}}$ é no máximo o número de vértices dos triângulos de $\mathcal{A}$ não-cobertos por nenhum triângulo de $\mathcal{O}$, ou seja, $e_{\mathcal{O}}^{\mathcal{T}} \leq 3\left|\mathcal{T}_{\mathcal{A}}\right|-o_{1}-2 o_{2}-3 o_{3}$. Portanto,

$$
e_{\mathcal{O}} \geq \max \left\{0,\left|\mathcal{E}_{\mathcal{O}}\right|-3\left|\mathcal{T}_{\mathcal{A}}\right|+o_{1}+2 o_{2}+3 o_{3}\right\} \text {. }
$$

Seja $G^{\prime}$ o grafo obtido removendo-se todos os vértices dos triângulos de $\mathcal{A}$, ou seja, $G^{\prime}:=$ $G-\bigcup_{T \in \mathcal{T}_{\mathcal{A}}} V_{T}$. Note que um emparelhamento de $G^{\prime}$ pode ser obtido tomando-se uma aresta de cada triângulo de $\mathcal{O}$ que tenha exatamente um vértice em comum com um triângulo de $\mathcal{A}$, e tomando-se as arestas de $\mathcal{E}_{\mathcal{O}}$ que não têm nenhum vértice em comum com um triângulo de $\mathcal{A}$. Portanto, como $\mathcal{E}_{\mathcal{A}}$ é um emparelhamento máximo de $G^{\prime}$, temos que $\left|\mathcal{E}_{\mathcal{A}}\right| \geq o_{1}+e_{\mathcal{O}}$. Deste fato e da desigualdade (5.12), temos que

$$
\left|\mathcal{E}_{\mathcal{A}}\right| \geq o_{1}+\max \left\{0,\left|\mathcal{E}_{\mathcal{O}}\right|-3\left|\mathcal{T}_{\mathcal{A}}\right|+o_{1}+2 o_{2}+3 o_{3}\right\}
$$

Da inequação acima e do fato (5.11), temos que o algoritmo de aproximação produz uma solução cujo valor é pelo menos

$3\left|\mathcal{T}_{\mathcal{A}}\right|+o_{1}+\max \left\{0,\left|\mathcal{E}_{\mathcal{O}}\right|-3\left|\mathcal{T}_{\mathcal{A}}\right|+o_{1}+2 o_{2}+3 o_{3}\right\}=3\left|\mathcal{T}_{\mathcal{A}}\right|+o_{1}+\max \left\{0,\left|\mathcal{E}_{\mathcal{O}}\right|-3\left|\mathcal{T}_{\mathcal{A}}\right|-o_{1}+o_{3}+2\left|\mathcal{T}_{\mathcal{O}}\right|\right\}$.

Consideramos agora a razão entre o valor de $\mathcal{O}$ e o valor de $\mathcal{A}$,

$$
r=\frac{3\left|\mathcal{T}_{\mathcal{O}}\right|+\left|\mathcal{E}_{\mathcal{O}}\right|}{3\left|\mathcal{T}_{\mathcal{A}}\right|+\left|\mathcal{E}_{\mathcal{A}}\right|}
$$

Analisamos os dois casos possíveis. 
a) $\left|\mathcal{E}_{\mathcal{O}}\right|-3\left|\mathcal{T}_{\mathcal{A}}\right|-o_{1}+o_{3}+2\left|\mathcal{T}_{\mathcal{O}}\right| \geq 0$. Neste caso, temos que

$$
r=\frac{3\left|\mathcal{T}_{\mathcal{O}}\right|+\left|\mathcal{E}_{\mathcal{O}}\right|}{3\left|\mathcal{T}_{\mathcal{A}}\right|+o_{1}+\left|\mathcal{E}_{\mathcal{O}}\right|-3\left|\mathcal{T}_{\mathcal{A}}\right|-o_{1}+o_{3}+2\left|\mathcal{T}_{\mathcal{O}}\right|} \leq \frac{3\left|\mathcal{T}_{\mathcal{O}}\right|+\left|\mathcal{E}_{\mathcal{O}}\right|}{2\left|\mathcal{T}_{\mathcal{O}}\right|+\left|\mathcal{E}_{\mathcal{O}}\right|} \leq \frac{3}{2}
$$

b) $\left|\mathcal{E}_{\mathcal{O}}\right|-3\left|\mathcal{T}_{\mathcal{A}}\right|-o_{1}+o_{3}+2\left|\mathcal{T}_{\mathcal{O}}\right|<0$. Neste caso, segue que $3\left|\mathcal{T}_{\mathcal{A}}\right|+o_{1}>\left|\mathcal{E}_{\mathcal{O}}\right|+o_{3}+2\left|\mathcal{T}_{\mathcal{O}}\right| \geq$ $\left|\mathcal{E}_{\mathcal{O}}\right|+2\left|\mathcal{T}_{\mathcal{O}}\right|$. Logo,

$$
r=\frac{3\left|\mathcal{T}_{\mathcal{O}}\right|+\left|\mathcal{E}_{\mathcal{O}}\right|}{3\left|\mathcal{T}_{\mathcal{A}}\right|+o_{1}}<\frac{3\left|\mathcal{T}_{\mathcal{O}}\right|+\left|\mathcal{E}_{\mathcal{O}}\right|}{2\left|\mathcal{T}_{\mathcal{O}}\right|+\left|\mathcal{E}_{\mathcal{O}}\right|} \leq \frac{3}{2}
$$

Observamos que a razão $3 / 2$ do algoritmo que descrevemos é justa, mesmo para grafos de grau máximo 4 (veja um exemplo na Figura 5.9).

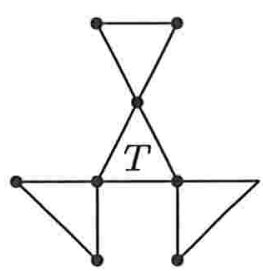

Figura 5.9: Se o algoritmo guloso de aproximação escolher inicialmente o triângulo $T$, então o valor devolvido pelo algoritmo é 6 . O valor da solução ótima é 9 .

Halldórsson [16] apresentou um algoritmo para a versão do problema de cobertura mínima por 3-conjuntos, com a condição adicional de que os conjuntos encontrados sejam dois a dois disjuntos, além de formarem uma cobertura do conjunto dos vértices do grafo. A análise do desempenho desse algoritmo baseia-se nos seguintes argumentos.

Seja $\mathcal{A}$ a cobertura devolvida pelo algoritmo de Halldórsson e seja $a_{i}$, para $i=1,2,3$, o número de conjuntos em $\mathcal{A}$ de tamanho $i$. Seja $\mathcal{O}$ uma solução ótima do problema e $o_{i}$, para $i=1,2,3$, o número de conjuntos em $\mathcal{O}$ de tamanho $i$. Claramente,

$$
a_{1}+2 a_{2}+3 a_{3}=b_{1}+2 b_{2}+3 b_{3} .
$$

A parte central consiste em mostrar que

$$
a_{1}+a_{2} \leq b_{1}+b_{2}+b_{3} .
$$

Substraindo (5.14) de (5.13), temos que $a_{2}+3 a_{3} \geq b_{2}+2 b_{3}$, e portanto,

$$
b_{2}+3 b_{3} \leq \frac{3}{2}\left(b_{2}+2 b_{3}\right) \leq \frac{3}{2}\left(a_{2}+3 a_{3}\right) .
$$

Ressaltamos que o algoritmo de Halldórsson é muito mais complicado do que o algoritmo proposto por Yuster. As idéias contidas na análise sugerida por Yuster guardam bastante semelhança com as idéias contidas na prova que apresentamos para o Teorema 5.3. Por outro lado, aproveitando as idéias de Yuster e juntando com outras novas, obtivemos alguns resultados preliminares interessantes para o problema do $\left\{K_{2}, \ldots, K_{r}\right\}$-empacotamento para $r>3$. Esses resultados farão parte de um artigo a ser redigido em breve. 


\section{Capítulo 6}

\section{Considerações finais}

Melhoramos a razão de aproximação para os problemas do empacotamento máximo de triângulos disjuntos nos vértices (VTP) e para o problema do empacotamento máximo de triângulos disjuntos nas arestas (ETP) em grafos de grau máximo limitado, ambos APX-completos [7]. Qualquer melhoria na razão de aproximação $\frac{3}{2}+\varepsilon$ para os problemas VTP ou ETP em grafos gerais seria muito interessante, bem como a melhoria dos limitantes inferiores para as razões de aproximação de VTP e ETP.

Uma classe de grafos para a qual não conhecemos a complexidade computacional do problema VTP é a classe dos grafos de intervalos. O problema VTP em grafos de intervalos é interessante tanto do ponto de vista prático, como do ponto de vista teórico. Como sabemos, esta classe de grafos contém a classe dos grafos de intervalos unitários, para a qual, como provamos, o problema VTP é polinomial. Também sabemos que os grafos de intervalos constituem uma subclasse dos grafos cordais para os quais VTP é NP-difícil [15].

$\mathrm{O}$ algoritmo de aproximação que apresentamos para o problema do $\mathrm{K}_{2}+\mathrm{K}_{3}$-empacotamento usa uma rotina para encontrar uma solução aproximada para o problema VTP. Do nosso resultado segue que qualquer melhora na razão de aproximação $\left(\frac{3}{2}+\varepsilon\right)$ para o problema VTP implica em uma melhor razão de aproximação para o problema do $K_{2}+K_{3}$-empacotamento. Seria interessante desenvolver outras abordagens para lidar com este problema que levem a melhores razões de aproximação, ou mostrar que isto é impossível.

Um outro problema que tem despertado nosso interesse é o problema do $\mathcal{F}$-empacotamento no caso em que $\mathcal{F}=\left\{K_{2}, K_{3}, \ldots, K_{r}\right\}$, para um $r$ fixo, e o objetivo é encontrar $H \subseteq G$ que seja uma união de grafos disjuntos nos vértices, cada um dos quais é isomorfo a um grafo de $\mathcal{F}$, e tal que $H$ tenha o maior número possível de arestas. Obtivemos alguns resultados de aproximação para este problema, que estão agora sendo refinados.

Recentemente, tomamos conhecimento de um problema de natureza semelhante, denominado de partição mínima em cliques cuja versão de otimização é a seguinte: dado um grafo $G=(V, E)$, encontre o menor $k$ tal que existe uma partição $\left(V_{1}, V_{2}, \ldots, V_{k}\right)$ de $V$ tal que, para cada $i \in\{1,2, \ldots, k\}$, o subgrafo $G\left[V_{i}\right]$ é uma clique. Cerioli et al. [8] provaram que este problema é MAX SNP-difícil para grafos cúbicos, e exibiram uma 5/4-aproximação para esta classe de grafos.

Encerramos este trabalho mencionando que há várias questões sobre problemas de $\mathcal{F}$ empacotamento, algumas pouco exploradas, e muitas ainda totalmente inexploradas. 


\section{Referências Bibliográficas}

[1] S. Arora. Reductions, codes, PCPs, and inapproximability. In Proc. 36th IEEE Symp. on Foundations of Computer Science, 1995.

[2] S. Arora, C. Lund, R. Motwani, M. Sudan, and M. Szegedy. Proof verification and the hardness of approximation problems. J. Assoc. Comput. Mach., 45(3):501-555, 1998.

[3] S. Arora and S. Safra. Probabilistic checking of proofs: a new characterization of NP. $J$. Assoc. Comput. Mach., 45(1):70-122, 1998.

[4] G. Ausiello, P. Crescenzi, G. Gambosi, V. Kann, A. Marchett-Spaccamela, and M. Protasi. Complexity and Approximation (Combinatorial Optimization Problems and Their Approximability Properties). Springer-Verlag, 1999.

[5] B. S. Baker. Approximation algorithms for NP-complete problems on planar graphs. $J$. Assoc. Comput. Mach., 41(1):153-180, 1994.

[6] P. Berman and T. Fujito. On approximation properties of the independent set problem for low degree graphs. Theory Comput. Syst., 32(2):115-132, 1999.

[7] A. Caprara and R. Rizzi. Packing triangles in bounded degree graphs. Inform. Process. Lett., 84(4):175-180, 2002.

[8] M. R Cerioli, L. Faria, T. O. Ferreira, C. A. J. Martinhon, F. Protti, and B. Reed. Partition into cliques for cubic graphs: planar case, complexity and appproximation algorithms. Manuscrito submetido.

[9] M. Chlebík and J. Chlebíková. Inapproximability results for bounded variants of optimization problems. In Lecture Notes in Computer Science 2751, pages 27-38. Springer, 2003.

[10] M. Chlebík and J. Chlebíková. On approximability of the independent set problem for low degree graphs. In Lecture Notes in Computer Science 3104, pages 47-56. Springer, 2004.

[11] G. Cornuéjols, D. Hartvigsen, and W. Pulleyblank. Packing subgraphs in a graph. Oper. Res. Lett., 1(4):139-143, 1981/82.

[12] G. Cornuéjols, D. Hartvigsen, and W. Pulleyblank. Packing subgraphs in a graph. Oper. Res. Lett., 4:139-143, 1982. 
[13] P. Erdős. On even subgraphs of graphs. Mat. Lapok, 18:283-288, 1967.

[14] R. L. Graham. Bounds for certain multiprocessor anomalies. Bell System Technical Journal, 45:1563-1581, 1966.

[15] V. Guruswami, C. Pandu Rangan, M. S. Chang, G. J. Chang, and C. K. Wong. The $K_{r}$-packing problem. Computing, 66(1):79-89, 2001.

[16] M. Halldórsson. Approximating $k$-set cover and complementary graph coloring. In Integer programming and combinatorial optimization, volume 1084 of Lecture Notes in Computer Science, pages 118-131. Springer, Berlin, 1996.

[17] P. Hell and D.G. Kirkpatrick. Star factors and star packings. Technical Report 82-6, School of Computing Science, Simon Fraser University, 1982.

[18] P. Hell and D.G. Kirkpatrick. On the complexity of general graph factor problems. SIAM J. Comput., 12(3):601-609, 1983.

[19] P. Hell and D.G. Kirkpatrick. Packings by cliques and by finite families of graphs. Discrete Math., 49:45-59, 1986.

[20] D. Hochbaum, editor. Approximation Algorithms for NP-hard Problems. PWS Publishing Company, 1997.

[21] I. Holyer. The NP-completeness of some edge-partition problems. SIAM J. Comput., 10(4):713-717, 1981.

[22] H. B. Hunt III, M. V. Marathe, V. Radhakrishnan, S. S. Ravi, D. J. Rosenkrantz, and R. E. Stearns. NC-approximation schemes for NP- and PSPACE-hard problems for geometric graphs. J. Algorithms, 26(2):238-274, 1998.

[23] C. A. J. Hurkens and A. Schrijver. On the size of systems of sets every $t$ of which have an SDR, with an application to the worst-case ratio of heuristics for packing problems. SIAM J. Discrete Math., 2(1):68-72, 1989.

[24] D. S. Johnson. Approximation algorithms for combinatorial problems. Journal of Computer and System Sciences, 9:256-278, 1974.

[25] R. M. Karp. On the computational complexity of combinatorial problems. Networks, 5(1):45-68, 1975.

[26] P. J. Looges and S. Olariu. Optimal greedy algorithms for indifference graphs. Comput. Math. Appl., 25(7):15-25, 1993.

[27] G. Manić and Y. Wakabayashi. Packing triangles in low degree graphs and indifference graphs. Discrete Mathematics and Theoretical Computer Science, Vol.AE 2005, pages 251-256, 2005. Disponível em http://www.ime.usp.br/ gocam/tr_pack.ps; versão completa submetida. 
[28] F. S. Roberts. Indifference graphs. In Proof Techniques in Graph Theory, pages 139-146. Academic Press, New York, 1969.

[29] V. Vazirani. Approximation Algorithms. Springer-Verlag, 2001.

[30] G. Yu and O. Goldschmidt. Local optimality and its application on independent sets for k-claw free graphs. Journal of Combinatorial Optimization, 1:151-164, 1997. 


\section{Índice Remissivo}

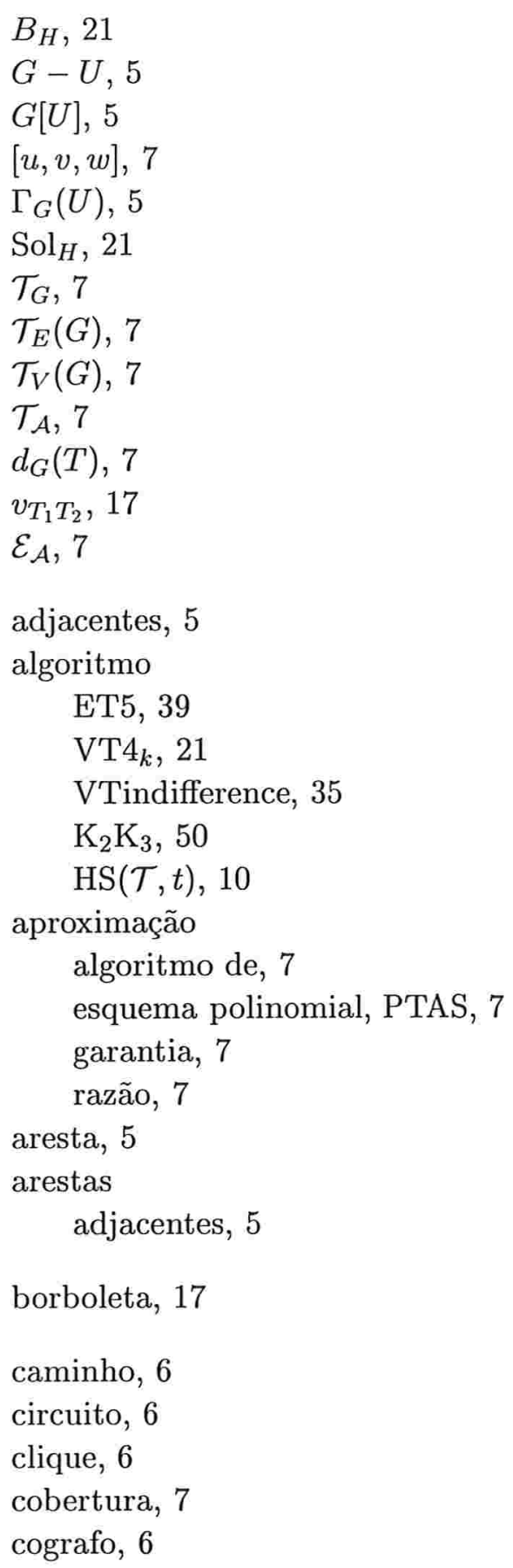

componente, 6

de atribuição, $\mathcal{X}_{i}, 45$

de teste, $\mathcal{C}_{j}, 45$

corda, 6

empacotamento

$t$-ótimo, 10

canônico, 45

de $\left\{K_{2}, K_{3}\right\}, 7$

valor de empacotamento de $\left\{K_{2}, K_{3}\right\}$,

7

emparelhamento, 5

estável, 5

grafo, 5

bipartido, 6

bipartido completo, 6

completo, 5

conexo, 6

cordal, 6

de intervalos, 6

de Hajós, 39

de interseção, 6

de intervalos unitários, 6

disco-unitário de $\lambda$-precisão, 6

indiferença, 6

irredundante, 7

livre de $k$-garras, 6

split, 6

vazio, 5

grau

de $T, d_{G}(T), 7$

de $v, d_{G}(v), 5$

máximo de $G, \Delta(G), 5$

incide em, 5

independente, 5 
interseção, $G \cap H, 5$

localmente ótimo, 17

ordem de $G,|G|, 5$

ordenação canônica, 35

Problema

de cobertura mínima por $k$-conjuntos, $k$-SC, 7

problema

APX-completo, 8

APX-difícil, 8

MAX2SAT3, 42

de Conjunto Independente Máximo, MIS,

6

de $\mathcal{F}$-empacotamento, 6

de Empacotamento Máximo de Triângulos

Disjuntos nas Arestas, ETP, ETP-

$k, 6$

de Empacotamento Máximo de Triângulos

Disjuntos nos Vértices, VTP, VTP-

$k, 6$

do $K_{2}+K_{3}$-empacotamento, 7

em APX, 7

procedimento

$\operatorname{Aceita}(\mathcal{T}), 21$

$\operatorname{Adota}(H), 21$

Redução(H), 21

Restauração(H), 22

redução

AP, 8

L, 8

sistema de representantes distintos, SRD, $t$-SRD, 7

subgrafo, 5

induzido, 5

triângulo, 5

união, $G \cup H, 5$

vértice, 5

isolado, 5

saturado, 16 vizinhança de $U, \Gamma_{G}(U), 5$

vizinhos, 5 